... (c) $200 \overline{4}$ American Chemical Society, J. Org. Chem., Postema jo040254t Supporting Info Page 1

\title{
REVISED
}

\section{RCM-Based Synthesis of a Variety of $\beta-C$ - Glycosides and their In Vitro Anti-Solid Tumor Activity}

\author{
Jared L. Piper, Russell L. Betts, Maarten H. D. Postema* \\ Department of Chemistry, Wayne State University, Detroit, MI 48202 \\ mposteml@HFHS.org \\ and
}

Frederick A. Valeriote, Halina Pietraszkewicz

Josephine Ford Cancer Center, Division of Hematology and Oncology

Department of Internal Medicine, Henry Ford Health System, Detroit, MI

fvaleril@hfhs.org

\section{Supporting Information}

\section{Table of Contents}

Synthesis of Esters 3b-3f, $3 \mathbf{h}-\mathbf{k}$ and $\beta-C$-Glycosides $6 \mathbf{b}-\mathbf{6 f}, \mathbf{6 h}-\mathbf{6 k}$

Synthesis of Glycolipids 17a-17c, 17d-17i, 17l

All proton NMR assignments were established through with the aid of the corresponding GCOSY and proton decoupling experiments. In general, a diagnostic proton was identified by its chemical shift and then that signal was correlated with adjacent protons. See included 2-D spectra for compounds $\mathbf{1 d}, \mathbf{3 g}$ and $6 \mathrm{~g}$ for details. 
(C) 2004 American Chemical Society, J. Org. Chem., Postema jo040254t Supporting Info Page 2

\section{ENTRY 1}

\section{RCM Examples}

3,4,6-tri- $O$-benzyl-1,2-dideoxy-5-O-(2-methoxyphenylacetyl)-D-arabino-hex-1-enitol (3a): ${ }^{1}$

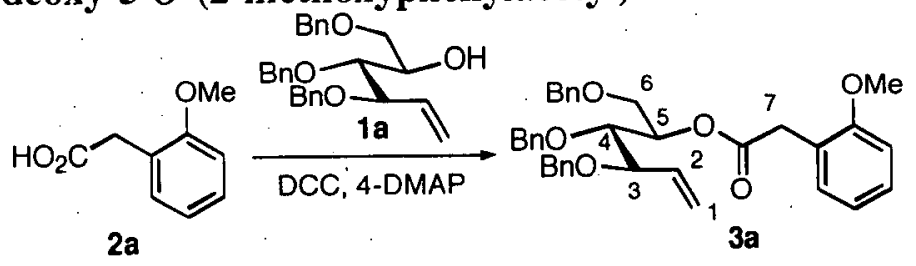

\section{$1^{\prime}-\left(3,4,6\right.$-tri- $O$-benzyl- $\beta$-D-glucosyl)-2-methoxytoluene (6a): ${ }^{2}$}

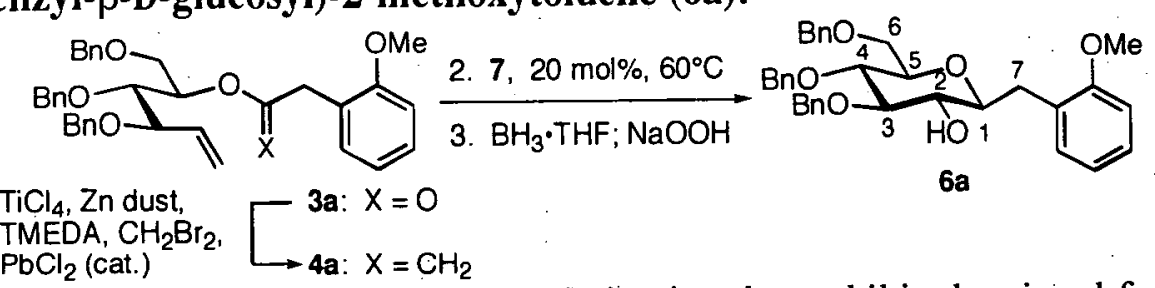

Acetylation of $6 \mathrm{a}$ gave the corresponding 2-acetyl derivative that exhibited a signal for $H-2$ at $4.94 \mathrm{ppm}$ (dd, $1 \mathrm{H}, J=8.8,8.8 \mathrm{~Hz}, \mathrm{CHOAc}$ ) indicating an axial relationship between $H-1, H-2$ and $H$-3 thus confirming the stereochemical assignment.

\section{ENTRY 2}

Triflate (8b)

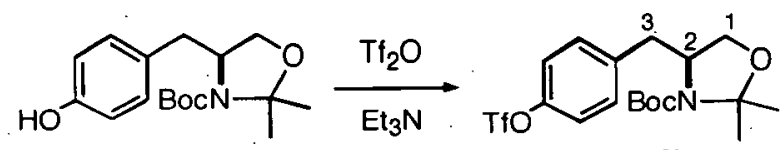

8b

To a stirring solution of the phenol $(450 \mathrm{mg}, 1.46 \mathrm{mmol})$, triethylamine $(0.22 \mathrm{~mL}, 1.61 \mathrm{mmol})$, and $\mathrm{CH}_{2} \mathrm{Cl}_{2}(6.0 \mathrm{~mL})$ cooled to $0^{\circ} \mathrm{C}$ was added $\mathrm{Tf}_{2} \mathrm{O}(0.27 \mathrm{~mL}, 1.61 \mathrm{mmol})$ dropwise. After the addition was complete, 4-DMAP ( $30 \mathrm{mg}, 0.25 \mathrm{mmol}$ ) was added, and stirred for $1 \mathrm{~h}$ at room temperature. The reaction mixture was poured into $\mathrm{H}_{2} \mathrm{O}(15 \mathrm{~mL})$, and extracted with $\mathrm{Et}_{2} \mathrm{O}(3 \times 25 \mathrm{~mL})$, washed with $\mathrm{H}_{2} \mathrm{O}(15 \mathrm{~mL})$, $10 \% \mathrm{HCl}(2 \times 15 \mathrm{~mL}), \mathrm{H}_{2} \mathrm{O}(15 \mathrm{~mL})$, and brine $(15 \mathrm{~mL})$. The organic extracts were dried over $\mathrm{MgSO}_{4}$, filtered and concentrated in vacuo to give $8 \mathbf{b}(622 \mathrm{mg}, 97 \%)$. The product $8 \mathbf{b}$ was used without further purification $\left(\mathrm{R}_{\mathrm{f}}=0.42\right.$, TLC, silica, $10 \% \mathrm{Et}_{2} \mathrm{O}$-hexanes; $\left.{ }^{1} \mathrm{H} \mathrm{NMR}, 500 \mathrm{MHz}\right){ }^{1} \mathrm{H} \mathrm{NMR}\left(500 \mathrm{MHz}, \mathrm{CDCl}_{3}\right.$, rotamers) $\delta 7.31(\mathrm{~d}, 1 \mathrm{H}, J=8.5 \mathrm{~Hz}, \mathrm{Ar} H), 7.26(\mathrm{~d}, 1 \mathrm{H}, J=8.5 \mathrm{~Hz}, \mathrm{Ar} H), 7.21(\mathrm{~d}, 1 \mathrm{H}, J=8.5 \mathrm{~Hz}, \mathrm{Ar} H)$, 7.18 (d, $1 \mathrm{H}, J=9.0 \mathrm{~Hz}, \mathrm{Ar} H), 4.09$ (dddd, $1 \mathrm{H}, J=3.5,3.5,3.5,3.5 \mathrm{~Hz}, H-2), 3.84-3.79$ (m, $1 \mathrm{H}, H-1$ ), $3.71(\mathrm{~d}, 1 \mathrm{H}, J=9.5 \mathrm{~Hz}, H-1), 3.17(\mathrm{dd}, 1 \mathrm{H}, J=13.0,2.5 \mathrm{~Hz}, H-3), 2.77-2.69(\mathrm{~m}, 1 \mathrm{H}, H-3), 1.61(\mathrm{~s}, 3 \mathrm{H}$, $\left.\mathrm{CH}_{3}\right), 1.50\left(\mathrm{~s}, 9 \mathrm{H}, 3 \times \mathrm{CH}_{3}\right), 1.45\left(\mathrm{~s}, 3 \mathrm{H}, \mathrm{CH}_{3}\right) ;{ }^{13} \mathrm{C} \mathrm{NMR}\left(125 \mathrm{MHz}, \mathrm{CDCl}_{3}\right.$, rotamers) $\delta 152.1,148.2$, $139.1,131.2,121.3,117.4,93.7,80.3,65.8,58.8,38.0,28.4,27.4,24.4$.

Styrene (9b)

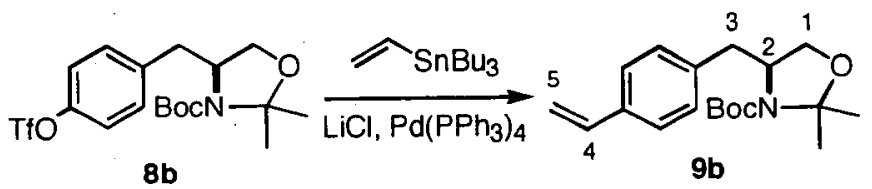

\footnotetext{
${ }^{1}$ For full experimental and spectral data listing for this compound please see the supporting information corresponding to: Postema, M. H. D.; Piper, J. L. Org. Lett. 2003, 5, 1721-1723.
} 
(C) 2004 American Chemical Society, J. Org. Chem., Postema jo040254t Supporting Info Page 3

Crude $8 \mathbf{b}$ (600 mg, $1.36 \mathrm{mmol})$ was re-dissloved in THF (15 mL) and charged into a round bottom flask containing $\mathrm{LiCl}(177 \mathrm{mg}, 4.18 \mathrm{mmol}),\left(\mathrm{Ph}_{3} \mathrm{P}\right)_{4} \mathrm{Pd}(31 \mathrm{mg}, 27.2 \mu \mathrm{mol})$, tributyl(vinyl)tin $(0.4 \mathrm{~mL}, 1.36$ $\mathrm{mmol})$ and THF $(10 \mathrm{~mL})$. The reaction mixture was degassed for $20 \mathrm{~min}$ and then heated to reflux for 11 $\mathrm{h}$, at which point TLC indicated complete conversion of the starting material. The mixture was cooled to room temperature and poured into ice-cold pentane $(25 \mathrm{~mL})$. The organic phase was washed with water $(10 \mathrm{~mL}), 10 \%$ ammonium hydroxide $(10 \mathrm{~mL})$, water $(10 \mathrm{~mL})$, brine $(10 \mathrm{~mL})$, dried over $\mathrm{MgSO}_{4}$, filtered and concentrated to give a viscous yellow oil. Flash chromatography of the residue over silica using $10 \rightarrow 15 \% \mathrm{Et}_{2} \mathrm{O}$-hexanes gave $9 \mathrm{~b}$ (350 mg, 76\% over 2 steps) as a pure $\left(\mathrm{R}_{\mathrm{f}}=0.35\right.$, TLC, silica, $15 \% \mathrm{Et}_{2} \mathrm{O}-$ hexanes; ' $\mathrm{H} \mathrm{NMR}, 500 \mathrm{MHz})$ white solid: m.p. $=84-85^{\circ} \mathrm{C} ;[\alpha]_{\mathrm{b}}^{26}=-14.5\left(c=1.00, \mathrm{CHCl}_{3}\right)$; FT-IR (neat) 2978, 2933, 2873, 1697, 1387, 1365, 1257, 1173, 1092, 1054, $856 \mathrm{~cm}^{-1} ;{ }^{1} \mathrm{H} \mathrm{NMR}\left(500 \mathrm{MHz}, \mathrm{CDCl}_{3}\right.$, all rotamer peaks reported) $\delta 7.38-7.32(\mathrm{~m}, 2 \mathrm{H}, 2 \times \mathrm{Ar} H), 7.21(\mathrm{~d}, 1 \mathrm{H}, J=8.0 \mathrm{~Hz}, \mathrm{Ar} H), 7.15(\mathrm{~d}, 1 \mathrm{H}, J=$ $7.5 \mathrm{~Hz}, \mathrm{Ar} H$ ), 6.68 (ddd, $1 \mathrm{H}, J=17,10.5,3.5 \mathrm{~Hz}, H-4), 5.72$ (dd, $1 \mathrm{H}, J=17.5,2.5 \mathrm{~Hz}, H-5$ ), 5.34-5.21 (dd, $1 \mathrm{H}, J=5.5,5.5 \mathrm{~Hz}, H-5$ ), 4.01 (dd, $1 \mathrm{H}, J=5.0,5.0 \mathrm{~Hz}, 0.5 \mathrm{H}, H-2$ ), 3.96 (dd, $1 \mathrm{H}, J=5.0,5.0 \mathrm{~Hz}$, $0.5 \mathrm{H}, H-2), 3.79-3.73(\mathrm{~m}, 2 \mathrm{H}, 2 \times H-1), 3.21(\mathrm{dd}, 0.5 \mathrm{H}, J=11,4.0 \mathrm{~Hz}, H-3), 2.65$ (dd, $0.5 \mathrm{H}, J=12.5$ $\mathrm{Hz}, H-3), 1.64\left(\mathrm{~s}, 1.5 \mathrm{H}, \mathrm{C}\left(\mathrm{CH}_{3}\right)_{2}\right), 1.55\left(\mathrm{~s}, 1.5 \mathrm{H}, \mathrm{C}\left(\mathrm{CH}_{3}\right)_{2}\right), 1.53\left(\mathrm{~s}, 9 \mathrm{H}, \mathrm{C}\left(\mathrm{CH}_{3}\right)_{3}\right), 1.50(\mathrm{~s}, 1.5 \mathrm{H}$, $\left.\mathrm{C}\left(\mathrm{CH}_{3}\right)_{2}\right), 1.46\left(\mathrm{~s}, 1.5 \mathrm{H}, \mathrm{C}\left(\mathrm{CH}_{3}\right)_{2}\right) ;{ }^{13} \mathrm{C} \mathrm{NMR}\left(125 \mathrm{MHz} \mathrm{CDCl}_{3}\right.$, data for only one rotamer reported) $\delta$ $151.6,138.1,136.4,135.9,129.4,126.4,113.5,94.0,79.7,66.0,59.1,39.3,29.7,28.5,26.9,23.2$; HRMS (FAB) calcd for $\mathrm{C}_{19} \mathrm{H}_{27} \mathrm{NO}_{3} \mathrm{Na}(\mathrm{M})^{+} 340.1883$, found 340.1887 .

Alcohol (10b)

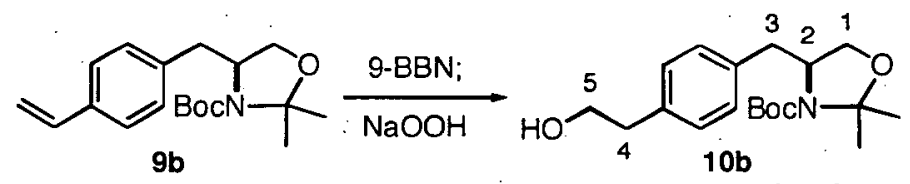

9-BBN (8.7 mL, $4.35 \mathrm{mmol}, 0.5 \mathrm{M}$ in THF) was added dropwise over $30 \mathrm{~min}$ to a cool $\left(0^{\circ} \mathrm{C}\right) \mathrm{THF}(10$ $\mathrm{mL})$ solution of $9 \mathrm{~b}(230 \mathrm{mg}, 0.73 \mathrm{mmol})$ and stirred for $1 \mathrm{~h}$, warmed to room temperature and stirred was for an additional $4 \mathrm{~h}$. The solution was cooled to $0{ }^{\circ} \mathrm{C}$ and $\mathrm{NaOH}(10 \mathrm{~mL}, 1 \mathrm{M}$ solution, $10 \mathrm{mmol})$ was added followed by $\mathrm{H}_{2} \mathrm{O}_{2}\left(10 \mathrm{~mL}, 0.1 \mathrm{mmol}, 30 \%\right.$ in $\left.\mathrm{H}_{2} \mathrm{O}\right)$. After warming to room temperature and an additional $2 \mathrm{~h}$ at room temperature, the biphasic mixture was treated with saturated $\mathrm{Na}_{2} \mathrm{~S}_{2} \mathrm{O}_{3}(3 \mathrm{~mL})$ and most of the THF was removed in vacuo. The aqueous mixture was then extracted with EtOAc $(3 \times 25$ $\mathrm{mL}$ ) and the combined organic extracts were washed with brine, dried over $\mathrm{MgSO}_{4}$ and filtered. Flash chromatography of the residue over silica using $25 \rightarrow 35 \%$ EtOAc-hexanes gave alcohol $10 \mathrm{~b}(225 \mathrm{mg}$, 93\%) as a pure $\left(R_{f}=0.30, T L C\right.$, silica, $35 \%$ EtOAc-hexanes; ${ }^{1} \mathrm{H}$ NMR, $\left.400 \mathrm{MHz}\right)$ white solid: m.p. $=66-$ $67^{\circ} \mathrm{C} ;[\alpha]^{26}=-20.8\left(c=1.00, \mathrm{CHCl}_{3}\right)$; FT-IR (neat) 3430 (br), 2978, 2932, 2872, 1696, 1454, 1389, 1365, $1257,1172,1093,1052,855,768 \mathrm{~cm}^{-1} ;{ }^{1} \mathrm{H}$ NMR (400 MHz, $\mathrm{CDCl}_{3}$, rotamers) $\delta 7.22-7.11(\mathrm{~m}, 4 \mathrm{H}, 4 \mathrm{x}$ ArH), 3.98-3.92 (m, $1 \mathrm{H}, H-2), 3.87-3.81(\mathrm{~m}, 2 \mathrm{H}, 2 \times H-5), 3.79-3.72(\mathrm{~m}, 2 \mathrm{H}, 2$ x H-4), 3.09-3.03 (m, 1 $\mathrm{H}, H-1), 2.87-2.80(\mathrm{~m}, 2 \mathrm{H}, 2 \times H-3), 2.67-2.58(\mathrm{~m}, 1 \mathrm{H}, H-1), 1.63\left(\mathrm{~s}, 3 \mathrm{H}, \mathrm{C}\left(\mathrm{CH}_{3}\right)_{2}\right), 1.52(\mathrm{~s}, 9 \mathrm{H}$, $\left.\mathrm{C}\left(\mathrm{CH}_{3}\right)_{3}\right), 1.49\left(\mathrm{~s}, 3 \mathrm{H}, \mathrm{C}\left(\mathrm{CH}_{3}\right)_{2}\right) ;{ }^{13} \mathrm{C} \mathrm{NMR}\left(125 \mathrm{MHz}, \mathrm{CDCl}_{3}\right.$, rotamers) $\delta 136.6,129.7,129.5,129.3$, 129.1 , 94.4, 94.0, 79.7, 66.0, 63.6, 59.2, 39.2, 38.7, 28.6, 26.9, 23.2; HRMS (FAB) calcd for $\mathrm{C}_{19} \mathrm{H}_{29} \mathrm{NO}_{4} \mathrm{Na}(\mathrm{M})^{+} 358.1989$, found 358.1987.

Acid (2b)

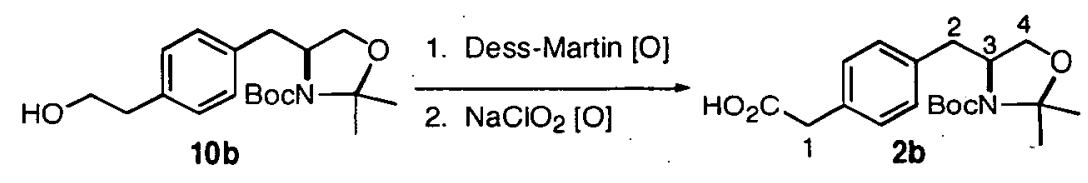


(C) 2004 American Chemical Society, J. Org. Chem., Postema jo040254t Supporting Info Page 4

Dess-Martin periodane $(704 \mathrm{mg}, 1.66 \mathrm{mmol})$ was added in one portion to a solution of alcohol $10 \mathrm{~b}$ (186 $\mathrm{mg}, 0.55 \mathrm{mmol})$ in $\mathrm{CH}_{2} \mathrm{Cl}_{2}(9 \mathrm{~mL})$ at ambient temperature. After $1 \mathrm{~h}$, the reaction mixture was treated with saturated $\mathrm{NaHSO}_{3}(0.2 \mathrm{~mL})$ followed by $\mathrm{NaHCO}_{3}(10 \mathrm{~mL})$ and the aqueous layer was extracted with $\mathrm{Et}_{2} \mathrm{O}(3 \times 10 \mathrm{~mL})$. The combined organic extracts were dried over $\mathrm{MgSO}_{4}$, filtered and concentrated to give a crude oil $(183 \mathrm{mg}, 0.55 \mathrm{mmol}, \sim 100 \%)$ that was taken up in $t$ - $\mathrm{BuOH}(7.5 \mathrm{~mL})$ and $\mathrm{H}_{2} \mathrm{O}(0.75 \mathrm{~mL})$. 2-Methyl-2-butene ( $310 \mu \mathrm{L}, 3.69 \mathrm{mmol}), \mathrm{NaClO}_{2}(65 \mathrm{mg}, 0.72 \mathrm{mmol})$ and $\mathrm{KH}_{2} \mathrm{PO}_{4}(86 \mathrm{mg}, 0.72 \mathrm{mmol})$ were then sequentially added to this suspension and stirring was continued for $12 \mathrm{~h}$ at ambient temperature. The organic phase was removed in vacuo and the aqueous phase acidified using $1 \mathrm{~N} \mathrm{HCl}(2$ $\mathrm{mL}, 2 \mathrm{mmol})$, extracted with EtOAc $(5 \times 15 \mathrm{~mL})$, washed with brine, dried over $\mathrm{MgSO}_{4}$ and concentrated. Flash chromatography of the residue over silica using $40 \rightarrow 45 \%$ EtOAc-hexanes- $0.5 \%$ AcOH gave acid $2 \mathbf{b}(178 \mathrm{mg}, 93 \%$ over 2 steps $)$ as a pure $\left(\mathrm{R}_{\mathrm{f}}=0.25\right.$, TLC, silica, $40 \%$ EtOAc-hexanes $(0.5 \% \mathrm{AcOH}) ;{ }^{1} \mathrm{H}$ NMR, $500 \mathrm{MHz})$ white solid: m.p. $=89-90^{\circ} \mathrm{C} ;[\alpha]_{\mathrm{D}}^{26}=-9.3\left(c=1.00, \mathrm{CHCl}_{3}\right)$; FT-IR (neat) 3200-3000. (br) 2978, 2925, 1703, 1407, 1390, 1257, 1168, 1094, 1016, $742 \mathrm{~cm}^{-1} ;{ }^{1} \mathrm{H} \mathrm{NMR} \mathrm{(500} \mathrm{MHz,} \mathrm{CDCl}_{3}$, only set of rotamer signals reported) $\delta 10.30\left(\mathrm{bs}, 1 \mathrm{H}, \mathrm{CO}_{2} H\right), 8.05(\mathrm{~d}, 1 \mathrm{H}, J=7.5, \mathrm{ArH}), 7.44(\mathrm{dd}, 1 \mathrm{H}, J=$ 7.5, 1.0 Hz, ArH), 7.22-7.16 (m, $2 \mathrm{H}, \mathrm{ArH}), 4.00-3.94(\mathrm{~m}, 1 \mathrm{H}, H-3), 3.80-3.74$ (m, $2 \mathrm{H}, 2$ x H-4), 3.69$3.63(\mathrm{~m}, 2 \mathrm{H}, 2 \times H-1), 3.08(\mathrm{dd}, 1 \mathrm{H}, J=13.0,1.0 \mathrm{~Hz}, H-2), 2.69-2.62(\mathrm{~m}, 1 \mathrm{H}, H-2), 1.65(\mathrm{~s}, 3 \mathrm{H}$, $\left.\mathrm{C}\left(\mathrm{CH}_{3}\right)_{2}\right), 1.52\left(\mathrm{~s}, 9 \mathrm{H}, \mathrm{C}\left(\mathrm{CH}_{3}\right)_{3}\right), 1.50\left(\mathrm{~s}, 3 \mathrm{H}, \mathrm{C}\left(\mathrm{CH}_{3}\right)_{2}\right) ;{ }^{13} \mathrm{C} \mathrm{NMR}\left(125 \mathrm{MHz}, \mathrm{CDCl}_{3}\right.$, only one set of rotamer signals reported) $\delta 171.1,153.0,141.9,137.6,133.4,129.7,129.6,128.0,94.6,66.1,59.1,40.7$, 29.7, 28.6, 26.9, 23.2; HRMS (FAB) calcd for $\mathrm{C}_{19} \mathrm{H}_{27} \mathrm{NO}_{5} \mathrm{Na}(\mathrm{M})^{+} 372.1781$, found 372.1789.

DCC-mediated esterification exemplified by the preparation of ester (3b)

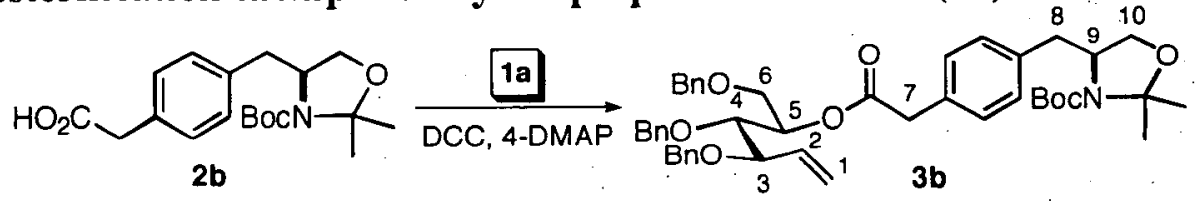

4-DMAP (44 mg, $0.36 \mathrm{mmol})$ and DCC ( $223 \mathrm{mg}, 1.08 \mathrm{mmol})$ were added in one portion to a dry solution of acid $2 \mathbf{b}(300 \mathrm{mg}, 0.86 \mathrm{mmol})$ and olefin alcohol $1 \mathbf{a}^{2}$ (300 mg, $\left.0.72 \mathrm{mmol}\right)$ in dry $\mathrm{CH}_{2} \mathrm{Cl}_{2}(5 \mathrm{~mL})$. The resulting solution was then stirred for $18 \mathrm{~h}$ at ambient temperature, at which point TLC $\left(25 \% \mathrm{Et}_{2} \mathrm{O}\right.$ hexanes) showed the reaction was complete. The reaction mixture was diluted with ether ( $20 \mathrm{~mL})$ and filtered by gravity through cotton to remove most of the formed dicyclohexylurea. The resulting organic solution was washed with $\mathrm{NH}_{4} \mathrm{Cl}(1 \times 30 \mathrm{~mL})$, dried and concentrated under reduced pressure. Flash chromatography of the residue over silica using $15 \rightarrow 25 \% \mathrm{Et}_{2} \mathrm{O}$-hexanes gave $\mathbf{3 b}(502 \mathrm{mg}, 93 \%)$ as a pure $\left(\mathrm{R}_{\mathrm{f}}=0.41\right.$, TLC, silica, $25 \% \mathrm{Et}_{2} \mathrm{O}$-hexanes; ' $\left.\mathrm{H} \mathrm{NMR}\left(400 \mathrm{MHz}, \mathrm{CDCl}_{3}\right)\right)$ yellow oil: $[\alpha]_{\mathrm{D}}^{26}=-20.0(c=$ $1.00, \mathrm{CHCl}_{3}$ ); FT-IR (neat) 2978, 2930, 2868, 1736, 1697, 1388, 1255 1092, 1072, 737, $698 \mathrm{~cm}^{-1} ;{ }^{1} \mathrm{H}$ NMR (400 MHz, $\mathrm{CDCl}_{3}$, only set of rotamer signals reported) $\delta 7.29-7.23(\mathrm{~m}, 4 \mathrm{H}, \mathrm{ArH}), 7.19-7.00(\mathrm{~m}$, $14 \mathrm{H}, \mathrm{Ar} H), 6.94-6.90(\mathrm{~m}, 1 \mathrm{H}, \mathrm{Ar} H), 5.84$ (ddd, $1 \mathrm{H}, J=18,10.5,7.5 \mathrm{~Hz}, H-2), 5.61$ (br d, $1 \mathrm{H}, J=5.2$ $\mathrm{Hz}, H-5), 5.09$ (d, $1 \mathrm{H}, J=18 \mathrm{~Hz}, H-1), 5.01(\mathrm{~d}, 1 \mathrm{H}, J=10.8 \mathrm{~Hz}, H-1), 4.59$ (d, $1 \mathrm{H}, J=11.2 \mathrm{~Hz}$, $\left.\mathrm{OCH}_{2} \mathrm{Ph}\right), 4.50\left(\mathrm{~d}, 1 \mathrm{H}, J=11.2 \mathrm{~Hz}, \mathrm{OCH}_{2} \mathrm{Ph}\right), 4.42\left(\mathrm{~d}, 1 \mathrm{H}, J=12 \mathrm{~Hz}, \mathrm{OCH}_{2} \mathrm{Ph}\right), 4.25-4.14(\mathrm{~m}, 2 \mathrm{H}, 2 \mathrm{x}$ $\left.\mathrm{OCH}_{2} \mathrm{Ph}\right), 4.06\left(\mathrm{~d}, 1 \mathrm{H}, J=11.2 \mathrm{~Hz}, \mathrm{OCH}_{2} \mathrm{Ph}\right), 3.91-3.82(\mathrm{~m}, 2 \mathrm{H}, H-3, H-4), 3.81-3.75(\mathrm{~m}, 2 \mathrm{H}, 2 \times H-6)$, 3.74-3.68 (m, $1 \mathrm{H}, H-9$ ), 3.54 (br d, $1 \mathrm{H}, J=9.0 \mathrm{~Hz}, H-7), 3.41-3.26$ (m, $3 \mathrm{H}, H-7,2 \times H-10), 3.02$ (dd, 1 $\mathrm{H}, J=10.5,1.0 \mathrm{~Hz}, H-8), 2.57(\mathrm{ddd}, 1 \mathrm{H}, J=23,13.5,12 \mathrm{~Hz}, H-8), 1.78\left(\mathrm{~s}, 3 \mathrm{H}, \mathrm{C}\left(\mathrm{CH}_{3}\right)_{2}\right), 1.53(\mathrm{~s}, 3 \mathrm{H}$, $\left.\mathrm{C}\left(\mathrm{CH}_{3}\right)_{2}\right), 1.43\left(\mathrm{~s}, 9 \mathrm{H}, \mathrm{C}\left(\mathrm{CH}_{3}\right)_{3}\right) ;{ }^{13} \mathrm{C}$ NMR $\left(100 \mathrm{MHz}, \mathrm{CDCl}_{3}\right) \delta 170.7,129.7,129.6,129.5,129.4,128.4$, $128.4,128.3,128.3,128.2,128.1,128.0,127.9,127.8,127.7,127.6,127.5,119.0,80.4,80.2,75.0,74.1$,

2 Freeman, F.; Robarge, K. D. Carbohydr. Res. 1987, 171, 1-11. 
(C) 2004 American Chemical Society, J. Org. Chem., Postema jo040254t Supporting Info Page 5

Postema, Piper, Betts, Valeriote and Pietraszkewicz RCM-Based Synthesis of a Variety of $\beta$ - $C$-Glycosides and their In Vitro Anti-Solid Tumor Activity

73.3, 73.2, 73.0, 70.8, 70.6, 70.3, 67.9, 59.2, 41.0, 28.6, 28.4, 23.2; HRMS (FAB) calcd for $\mathrm{C}_{46} \mathrm{H}_{55} \mathrm{O}_{8} \mathrm{Na}$ $(\mathrm{M})^{+} 772.3820$, found 772.3825 .

General grocedure for the three-step protocol exemplified by the preparation of $C$-glycoside (6b)
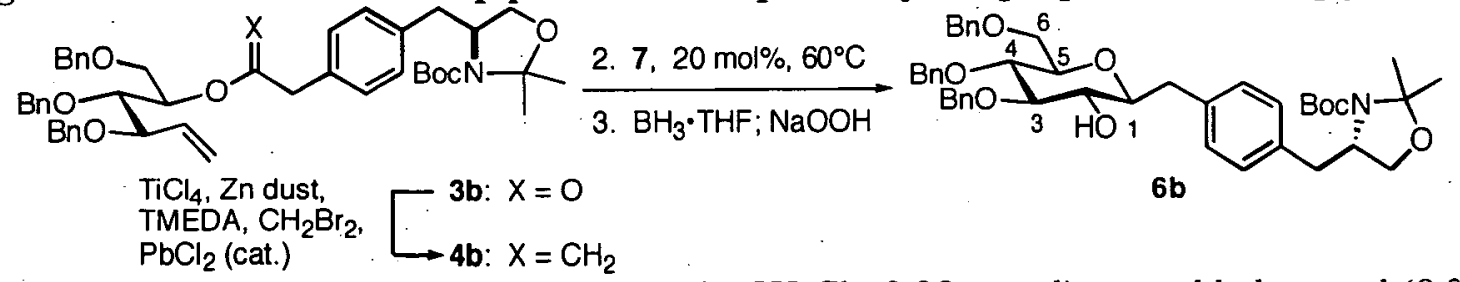

A solution of titanium tetrachloride $\left(1.19 \mathrm{~mL}, 2 \mathrm{M}\right.$ in $\left.\mathrm{CH}_{2} \mathrm{Cl}_{2}, 2.38 \mathrm{mmol}\right)$ was added to cool $\left(0^{\circ} \mathrm{C}\right) \mathrm{THF}$ $(2 \mathrm{~mL})$. The resulting mixture was stirred for 30 minutes at which point TMEDA $(0.69 \mathrm{~mL}, 4.59 \mathrm{mmol})$ was added in one portion. The resulting yellow-brown suspension was allowed to warm to ambient temperature and stirred for 30 minutes. At this point, zinc dust (339 mg, $5.18 \mathrm{mmol})$ and lead (II) chloride ( $4 \mathrm{mg}, 0.014 \mathrm{mmol})$ were added in one portion and stirring at ambient temperature was continued for $10 \mathrm{~min}$. A solution of ester $3 \mathrm{~b} \cdot(100 \mathrm{mg}, 0.14 \mathrm{mmol})$ and dibromomethane $(91 \mu \mathrm{L}, 1.3 \mathrm{mmol})$ in THF ( $2 \mathrm{~mL}$ ) was then added via cannula to the reaction flask in one portion. The mixture was stirred at $60^{\circ} \mathrm{C}$ for 1 hour, cooled to $0^{\circ} \mathrm{C}$ and then quenched by the addition of saturated potassium carbonate $(1.0 \mathrm{~mL})$. The resulting mixture was stirred for 30 minutes (while warming to ambient temperature), diluted with ether $(20 \mathrm{~mL})$ and stirred vigorously for 15 minutes. The resulting mixture was filtered through basic alumina using $3 \%$ triethylamine-ether as the eluent. The greenish-blue precipitate that resulted was crushed (mortar and pestle) and thoroughly extracted by vigorous stirring over diethyl ether (15-20 mL) for 30 minutes. The combined ethereal extracts were concentrated in vacuo, azeotroped with benzene $(3 \mathrm{x}$ $20 \mathrm{ml})$ and re-dissolved in dry toluene $(14 \mathrm{~mL})$. After the crude enol ether was degassed thoroughly under nitrogen atmosphere for 20 minutes, the first aliquot (1/4) of catalyst $7(30 \mathrm{mg}, 0.158 \mathrm{mmol}, 25$ mol\%) was added and heated to $60^{\circ} \mathrm{C}$. The remaining catalyst was added in three more portions over the next three hours and upon completion, the solution was cooled to $0^{\circ} \mathrm{C}, \mathrm{BH}_{3} \cdot \mathrm{THF}(1.4 \mathrm{~mL}, 1 \mathrm{M}$ in THF, $1.4 \mathrm{mmol}$ ) was added to the cooled solution and the resulting mixture was stirred at this temperature for three hours until TLC indicated the reaction was complete. $\mathrm{NaOH}(10 \mathrm{~mL}, 1 \mathrm{M})$ and hydrogen peroxide (10 $\mathrm{mL}, 30 \%$ in water) were added sequentially dropwise, and the solution was allowed to warm to room temperature over 2 hours at which point TLC showed a clean product spot. The solution was extracted with ether $(3 \times 15 \mathrm{~mL})$ and the combined ethereal extracts were washed with brine $(1 \times 20 \mathrm{~mL})$, dried over magnesium sulfate, filtered and concentrated in vacuo. Flash chromatography of the residue over silica using $10 \rightarrow 20 \%$ EtOAc-hexanes gave $6 \mathbf{b}\left(59 \mathrm{mg}, 59 \%\right.$ over 3 steps) as a pure $\left(R_{\mathrm{f}}=0.32\right.$, TLC, silica, 20\% EtOAc-hexanes; ${ }^{1} \mathrm{H}$ NMR $\left(400 \mathrm{MHz}, \mathrm{CDCl}_{3}\right)$ ) oil: $[\alpha]_{D}^{26}=+5.2\left(c=1.00, \mathrm{CHCl}_{3}\right) ; \mathrm{FT}$-IR (neat) $3463,2979,2929,2868,1695,1453,1388,1364,1257,1106,1094,855,734,697 \mathrm{~cm}^{-1}$; ${ }^{1} \mathrm{H}$ NMR (400 $\mathrm{MHz} \mathrm{CDCl}_{3}$, rotamers) $\delta 7.40-7.20(\mathrm{~m}, 17 \mathrm{H}, \mathrm{ArH}), 7.18-7.04(\mathrm{~m}, 2 \mathrm{H}, \mathrm{ArH}), 4.96(\mathrm{~d}, 1 \mathrm{H}, J=11.2 \mathrm{~Hz}$, $\left.\mathrm{OCH}_{2} \mathrm{Ph}\right), 4.79\left(\mathrm{~d}, 1 \mathrm{H}, \mathrm{J}=10.4 \mathrm{~Hz}, \mathrm{OCH}_{2} \mathrm{Ph}\right), 4.73\left(\mathrm{~d}, 1 \mathrm{H}, J=11.2 \mathrm{~Hz}, \mathrm{OCH}_{2} \mathrm{Ph}\right), 4.62(\mathrm{~d}, 1 \mathrm{H}, J=10.8$ $\left.\mathrm{Hz}, \mathrm{OCH}_{2} \mathrm{Ph}\right), 4.58-4.48\left(\mathrm{~m}, 2 \mathrm{H}, 2 \times \mathrm{OCH}_{2} \mathrm{Ph}\right), 3.97(\mathrm{~m}, 1 \mathrm{H}, H-9), 3.78-3.66(\mathrm{~m}, 4 \mathrm{H}, 2 \times H-6,2 \times H-$ 10), 3.59 (dd, $1 \mathrm{H}, J=9.6,9.6 \mathrm{~Hz}, H-4), 3.49$ (dd, $1 \mathrm{H}, J=8.8,8.8 \mathrm{~Hz}, H-3), 3.44-3.37(\mathrm{~m}, 2 \mathrm{H}, H-1, H-$ 5), 3.32 (dd, $1 \mathrm{H}, J=9.2,9.2 \mathrm{~Hz}, H-2), 3.20-3.02$ (m, $2 \mathrm{H}, H-7, H-8), 2.84-2.72$ (m, $1 \mathrm{H}, H-7$ ), 2.61 (dd, 1 $\mathrm{H}, J=11.2,11.2 \mathrm{~Hz}, \mathrm{H}-8), 1.65\left(\mathrm{~s}, 3 \mathrm{H}, \mathrm{C}\left(\mathrm{CH}_{3}\right)_{2}\right), 1.53\left(\mathrm{~s}, 9 \mathrm{H}, \mathrm{C}\left(\mathrm{CH}_{3}\right)_{3}\right), 1.50\left(\mathrm{~s}, 3 \mathrm{H}, \mathrm{C}\left(\mathrm{CH}_{3}\right)_{2}\right) ;{ }^{13} \mathrm{C}$ NMR (125 MHz, $\mathrm{CDCl}_{3}$, rotamers) $\delta 151.6,138.3,138.0,136.5,136.2,130.1,128.9,128.7,128.4,127.9$, $127.8,127.6,127.5,93.9,86.7,79.7,79.1,78.4,74.7,73.4,72.9,68.8,66.0,59.2,39.2,37.3,28.5,26.9$, 23.2; HRMS (FAB) calcd for $\mathrm{C}_{46} \mathrm{H}_{54} \mathrm{O}_{8} \mathrm{Na}(\mathrm{M})^{+} 760.3820$, found 760.3833 . 
(C) 2004 American Chemical Society, J. Org. Chem., Postema jo040254t Supporting Info Page 6

\section{ENTRY 3}

Vinyl (9c)
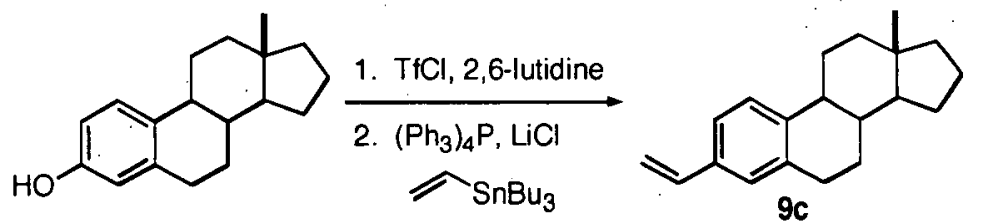

Triflyl chloride $(54 \mu \mathrm{L}, 0.51 \mathrm{mmol})$ was added dropwise to a stirred and cooled $\left(0{ }^{\circ} \mathrm{C}\right)$ solution of 3deoxyestrone $^{3}(120 \mathrm{mg}, 0.47 \mathrm{mmol})$ and triethylamine $(72 \mu \mathrm{L}, 0.51 \mathrm{mmol})$ in $\mathrm{CH}_{2} \mathrm{Cl}_{2}(1.5 \mathrm{~mL})$. After the addition was complete, 4-DMAP ( $3 \mathrm{mg}, 24.6 \mu \mathrm{mol})$ was added and stirring was continued for $1 \mathrm{~h}$ at ambient temperature. The reaction mixture was poured into water $(5 \mathrm{~mL})$, extracted with $\mathrm{Et}_{2} \mathrm{O}(3 \times 10$ $\mathrm{mL})$, washed with $\mathrm{H}_{2} \mathrm{O}(5 \mathrm{~mL}), 10 \% \mathrm{HCl}(2 \times 5 \mathrm{~mL}), \mathrm{H}_{2} \mathrm{O}(5 \mathrm{~mL})$ and brine $(5 \mathrm{~mL})$. The organic extracts were dried, filtered and concentrated in vacuo to provide a pale yellow oil (172 $\mathrm{mg}, 84 \%$ crude yield). A solution of the crude product in THF $(1 \mathrm{~mL})$ was added to a THF $(2 \mathrm{~mL})$ solution of $\mathrm{LiCl}(56 \mathrm{mg}, 1.32$ $\mathrm{mmol}),\left(\mathrm{Ph}_{3} \mathrm{P}\right)_{4} \mathrm{Pd}(10 \mathrm{mg}, 8.75 \mu \mathrm{mol})$ and tributyl(vinyl)tin $(0.13 \mathrm{ml}, 0.43 \mathrm{mmol})$. The reaction mixture was degassed for $20 \mathrm{~min}$ and heated to reflux for $11 \mathrm{~h}$, at which point TLC indicated complete conversion. The mixture was cooled to room temperature and poured into ice-cold pentane $(10 \mathrm{~mL})$. The organic phase was washed with water $(5 \mathrm{~mL}), 10 \%$ ammonium hydroxide $(5 \mathrm{~mL})$, water $(5 \mathrm{~mL})$, brine $(5 \mathrm{~mL})$, dried, filtered and concentrated to give a viscous yellow oil. Flash chromatography of the residue over silica gel using $10 \rightarrow 20 \% \mathrm{Et}_{2} \mathrm{O}$-hexanes gave $9 \mathrm{c}\left(95 \mathrm{mg}, 76 \%\right.$ over 2 steps) as a pure $\left(\mathrm{R}_{\mathrm{f}}=0.30\right.$; TLC, silica, $15 \% \mathrm{Et}_{2} \mathrm{O}$-hexanes; $\left.{ }^{2} \mathrm{H} \mathrm{NMR}, 500 \mathrm{MHz}\right)$ white solid: m.p. $=44-45^{\circ} \mathrm{C} ;[\alpha]_{D}=+102.4(c=1.00$, $\mathrm{CHCl}_{3}$ ); FT-IR (neat) 2922, 2866, 1706, 1498, 1451, 1376, 988, 897, $830 \mathrm{~cm}^{-1} ;{ }^{1} \mathrm{H}$ NMR $(500 \mathrm{MHz}$, $\left.\mathrm{CDCl}_{3}\right) \delta 7.29(\mathrm{~d}, 1 \mathrm{H}, J=8.0 \mathrm{~Hz}, \mathrm{ArH}), 7.22(\mathrm{~d}, 1 \mathrm{H}, J=8.0 \mathrm{~Hz}, \mathrm{ArH}), 7.15(\mathrm{~s}, 1 \mathrm{H}, \mathrm{ArH}), 6.69(\mathrm{dd}, 1 \mathrm{H}$, $J=17.5,11.0 \mathrm{~Hz}, \mathrm{C}=\mathrm{C}-H), 5.72(\mathrm{~d}, 1 \mathrm{H}, J=17.5 \mathrm{~Hz}, \mathrm{C}=\mathrm{CH}-H), 5.20(\mathrm{~d}, 1 \mathrm{H}, J=10.5 \mathrm{~Hz}, \mathrm{C}=\mathrm{CH}-H)$, 2.94-2.86(m, $2 \mathrm{H}, 2 \times \mathrm{CH}), 2.36-2.24(\mathrm{~m}, 2 \mathrm{H}, 2 \times \mathrm{CH}), 1.99-1.88(\mathrm{~m}, 2 \mathrm{H}, 2 \times \mathrm{CH}), 1.84-1.75(\mathrm{~m}, 1 \mathrm{H}$, $\mathrm{CH}), 1.75-1.63(\mathrm{~m}, 2 \mathrm{H}, 2 \times \mathrm{CH}), 1.60-1.50(\mathrm{~m}, 2 \mathrm{H}, 2 \times \mathrm{CH}), 1.46-1.34(\mathrm{~m}, 3 \mathrm{H}, 3 \times \mathrm{CH}), 1.34-1.23(\mathrm{~m}, 2$ $\mathrm{H}, 2 \times \mathrm{CH}), 1.22-1.14(\mathrm{~m}, 1 \mathrm{H}, \mathrm{CH}), 0.77\left(\mathrm{~s}, 3 \mathrm{H}, \mathrm{CH}_{3}\right) ;{ }^{13} \mathrm{C} \mathrm{NMR}\left(125 \mathrm{MHz}, \mathrm{CDCl}_{3}\right) \delta 140.6,136.9$, $136.7,134.8,126.8,125.5,123.4,112.8,53.7,44.6,41.0,40.5,39.0,38.8,29.7,28.1,26.6,25.2,20.6$, 17.5; HRMS (FAB) calcd for $\mathrm{C}_{20} \mathrm{H}_{26}(\mathrm{M})^{+} 266.2035$, found 266.2036.

Alcohol (10c)
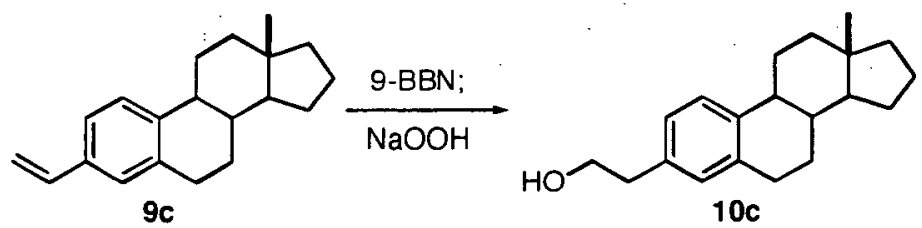

9-BBN (4.5 mL, $2.25 \mathrm{mmol}, 0.5 \mathrm{M}$ in THF) was added dropwise over $30 \mathrm{~min}$ to a cool $\left(0^{\circ} \mathrm{C}\right) \mathrm{THF}$ solution of the vinyl estrone derivative $9 \mathrm{c}(500 \mathrm{mg}, 1.88 \mathrm{mmol})$ dissolved in THF $(15 \mathrm{~mL})$ and the reaction was stirred for $1 \mathrm{~h}$ before warming to room temperature. Stirring was continued for an additional hour and $\mathrm{NaOH}\left(10 \mathrm{~mL}, 10 \mathrm{mmol}, 1 \mathrm{M}\right.$ solution), followed by $\mathrm{H}_{2} \mathrm{O}_{2}\left(10 \mathrm{~mL}, 30 \%\right.$ in $\left.\mathrm{H}_{2} \mathrm{O}\right)$ were added dropwise. After $2 \mathrm{~h}$ at room temperature, the biphasic mixture was treated with saturated $\mathrm{Na}_{2} \mathrm{SO}_{3}(3 \mathrm{~mL})$ and the organic phase was removed in vacuo. The aqueous mixture was extracted with EtOAc $(3 \times 25$ $\mathrm{mL}$ ), washed with brine, dried, filtered and concentrated. Flash chromatography of the residue over silica using $30 \% \mathrm{Et}_{2} \mathrm{O}$-hexanes gave $10 \mathrm{c}(508 \mathrm{mg}, 95 \%)$ as pure $\left(\mathrm{R}_{\mathrm{f}}=0.33\right.$, TLC, silica $30 \% \mathrm{Et}_{2} \mathrm{O}$-hexanes; ${ }^{1} \mathrm{H}$ NMR, $500 \mathrm{MHz})$ white needles: m.p. $=82-83{ }^{\circ} \mathrm{C} ;\left[\alpha b^{26}=+71.5\left(c=1.00, \mathrm{CHCl}_{3}\right)\right.$; FT-IR (neat) 3330 (br),

${ }^{3}$ Huang-Minlon, J. Am. Chem. Soc. 1949, 71, 3301-3303. 
(C) 2004 American Chemical Society, J. Org. Chem., Postema jo040254t Supporting Info Page 7

$2925,2867,1713,1500,1451,1376,1045,829,755,692 \mathrm{~cm}^{-1} ;{ }^{\prime} \mathrm{H}$ NMR $\left(500 \mathrm{MHz}, \mathrm{CDCl}_{3}\right) \delta 7.26(\mathrm{~d}, 1$ $\mathrm{H}, J=8.0 \mathrm{~Hz}, \operatorname{Ar} H), 7.00(\mathrm{~d}, 1 \mathrm{H}, J=7.5 \mathrm{~Hz}, \operatorname{Ar} H), 6.95(\mathrm{~s}, 1 \mathrm{H}, \operatorname{Ar} H), 3.88-3.82(\operatorname{app~t}, 2 \mathrm{H}, 2 \times \mathrm{OCH})$, 2.92-2.84 (m, $2 \mathrm{H}, 2 \times \mathrm{CH}), 2.84-2.78(\mathrm{~m}, 2 \mathrm{H}, 2 \times \mathrm{CH}), 2.34-2.20(\mathrm{~m}, 2 \mathrm{H}, 2 \times \mathrm{CH}), 1.96-1.85(\mathrm{~m}, 2 \mathrm{H}, 2$ x CH), 1.81-1.72(m, 1 H, CH), 1.72-1.60 (m, $2 \mathrm{H}, 2 \times \mathrm{CH}), 1.60-1.46(\mathrm{~m}, 3 \mathrm{H}, 3 \times \mathrm{CH}), 1.44-1.30(\mathrm{~m}, 2$ $\mathrm{H}, 2 \times \mathrm{CH}), 1.32-1.20(\mathrm{~m}, 2 \mathrm{H}, 2 \times \mathrm{CH}), 1.20-1.11(\mathrm{~m}, 1 \mathrm{H}, \mathrm{CH}), 0.75\left(\mathrm{~s}, 3 \mathrm{H}, \mathrm{CH}_{3}\right) ;{ }^{13} \mathrm{C}$ NMR $(125 \mathrm{MHz}$, $\left.\mathrm{CDCl}_{3}\right) \delta 139.0,137.0,135.3,129.6,126.2,125.7,63.7,53.6,44.4,41.1,40.5,39.0,38.8,38.6,29.7$, 28.1, 26.6, 25.2, 20.6, 17.5; HRMS (FAB) calcd for $\mathrm{C}_{20} \mathrm{H}_{28} \mathrm{ONa}(\mathrm{M})^{+} 307.2032$, found 307.2037.

Acid (2c)

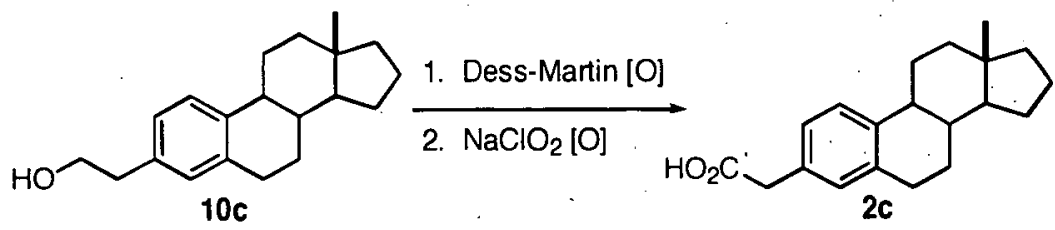

Dess-Martin periodane $(210 \mathrm{mg}, 0.5 \mathrm{mmol})$ was added in one portion to a stirred $\mathrm{CH}_{2} \mathrm{Cl}_{2}(8 \mathrm{~mL})$ solution of alcohol $10 \mathrm{c}$ (94 $\mathrm{mg}, 0.33 \mathrm{mmol}$ ) at ambient temperature. After $1 \mathrm{~h}$, the reaction mixture was treated sequentially with saturated $\mathrm{NaHSO}_{3}(0.5 \mathrm{~mL})$ and $\mathrm{NaHCO}_{3}(10 \mathrm{~mL})$ and the aqueous layer was extracted with $\mathrm{Et}_{2} \mathrm{O}(3 \times 10 \mathrm{~mL})$. The combined organic extracts were dried, filtered and concentrated to give a crude oil ( $90 \mathrm{mg}, 0.32 \mathrm{mmol}, 96 \%$ ) that was taken up in $t$ - $\mathrm{BuOH}(4.3 \mathrm{~mL})$ and $\mathrm{H}_{2} \mathrm{O}(0.43 \mathrm{~mL})$. 2-Methyl2-butene ( $185 \mu \mathrm{L}, 2.2 \mathrm{mmol}), \mathrm{NaClO}_{2}(39 \mathrm{mg}, 0.43 \mathrm{mmol})$ and $\mathrm{KH}_{2} \mathrm{PO}_{4}(52 \mathrm{mg}, 0.43 \mathrm{mmol})$ were then sequentially added to this suspension and stirring was continued for $12 \mathrm{~h}$ at ambient temperature. The organic phase was removed in vacuo and the aqueous phase acidified with $1 \mathrm{~N} \mathrm{HCl}(2 \mathrm{~mL})$, extracted with EtOAc $(5 \times 10 \mathrm{~mL})$, washed with brine, dried over $\mathrm{MgSO}_{4}$ and concentrated. Flash chromatography of the residue over silica using $25 \%$ EtOAc-hexanes- $0.5 \% \mathrm{AcOH}$ gave $2 \mathrm{c}(90 \mathrm{mg}, 91 \%$ over 2 steps) as a pure $\left(\mathrm{R}_{\mathrm{f}}=0.24\right.$, TLC, silica $25 \%$ EtOAc-hexanes $\left.(0.5 \% \mathrm{AcOH}) ;{ }^{1} \mathrm{H} \mathrm{NMR}, 500 \mathrm{MHz}\right)$ white solid: m.p. $=$ $127-128^{\circ} \mathrm{C} ;[\alpha]^{26}=+64.4\left(c=1.00, \mathrm{CHCl}_{3}\right)$; FT-IR (neat) $3400-2700$ (br) 2930, 2866, 1704, 1499, 1450, $1431,1408,1376,1358,1296,1217,1154,940 \mathrm{~cm}^{-1} ;{ }^{1} \mathrm{H}$ NMR $\left(500 \mathrm{MHz}, \mathrm{CDCl}_{3}\right) \delta 7.28(\mathrm{~d}, 1 \mathrm{H}, J=8.0$ $\mathrm{Hz}, \mathrm{Ar} H), 7.04$ (d, $1 \mathrm{H}, J=7.5 \mathrm{~Hz}, \mathrm{Ar} H), 7.01$ (s, $1 \mathrm{H}, \mathrm{ArH}), 3.60$ (app s, $2 \mathrm{H}, 2$ x C(=O)OCH $\left.2^{-}\right), 2.92-$ $2.85(\mathrm{~m}, 2 \mathrm{H}, 2 \times \mathrm{CH}), 1.97-1.87(\mathrm{~m}, 2 \mathrm{H}, 2 \times \mathrm{CH}), 1.82-1.74(\mathrm{~m}, 1 \mathrm{H}, \mathrm{CH}), 1.74-1.63(\mathrm{~m}, 2 \mathrm{H}, 2 \times \mathrm{CH})$, 1.59-1.50 (m, $2 \mathrm{H}, 2 \times \mathrm{CH}), 1.46-1.33(\mathrm{~m}, 3 \mathrm{H}, 3 \times \mathrm{CH}), 1.33-1.22(\mathrm{~m}, 2 \mathrm{H}, 2 \times \mathrm{CH}), 1.20-1.13(\mathrm{~m}, 1 \mathrm{H}$, $\mathrm{CH}), 0.75\left(\mathrm{~s}, 3 \mathrm{H}, \mathrm{CH}_{3}\right) ;{ }^{13} \mathrm{C}$ NMR $\left(125 \mathrm{MHz}_{\mathrm{CDCl}}\right) \delta 178.3,139.9,137.2,130.2,129.8,126.5,125.7$, 53.6, 44.3, 41.0, 40.6, 40.5, 38.8, 38.8, 29.6, 28.0, 26.5, 25.2, 20.5, 17.5; HRMS (FAB) calcd for $\mathrm{C}_{20} \mathrm{H}_{26} \mathrm{O}_{2} \mathrm{Na}(\mathrm{M})^{+} .321 .1825$, found 321.1827 .

\section{Ester (3c)}
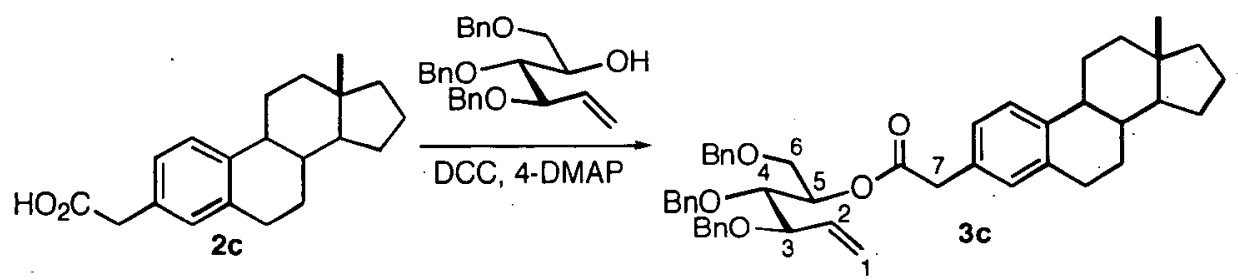

The general method for esterification was followed using: alcohol $1 \mathbf{a}^{4}$ (351 $\left.\mathrm{mg}, 0.84 \mathrm{mmol}\right)$, acid $\mathbf{2 c}(300$ $\mathrm{mg}, 1.01 \mathrm{mmol})$, DCC (260 mg, $1.26 \mathrm{mmol})$, 4-DMAP (51 mg, $0.42 \mathrm{mmol})$ and $\mathrm{CH}_{2} \mathrm{Cl}_{2}(6 \mathrm{~mL})$. Flash chromatography of the residue over silica using $15 \rightarrow 25 \% \mathrm{Et}_{2} \mathrm{O}$-hexanes gave $3 \mathrm{c}(490 \mathrm{mg}, 82 \%)$ as a pure

\footnotetext{
${ }^{4}$ Freeman, F.; Robarge, K. D. Carbohydr. Res. 1987, 17l, 1-11.
} 
(C) 2004 American Chemical Society, J. Org. Chem., Postema jo040254t Supporting Info Page 8

$\left(\mathrm{R}_{\mathrm{f}}=0.35\right.$, TLC, silica, $25 \%$ diethyl ether-hexanes; ${ }^{1} \mathrm{H}$ NMR $\left.\left(500 \mathrm{MHz}, \mathrm{CDCl}_{3}\right)\right)$ yellow oil: $[\alpha]_{b}^{26}=+50.6$ $\left(c=1.00, \mathrm{CHCl}_{3}\right.$ ); FT-IR (neat) 3063, 3030, 2920, 2866, 2360, 2342, 1732, 1497, 1453, 1251, 1066, 733, $696 \mathrm{~cm}^{-1}$; ${ }^{1} \mathrm{H}$ NMR $\left(500 \mathrm{MHz}, \mathrm{CDCl}_{3}\right) \delta 7.40-7.28(\mathrm{~m}, 15 \mathrm{H}, \mathrm{Ar} H), 7.25(\mathrm{~d}, 1 \mathrm{H}, J=7.5 \mathrm{~Hz}, \mathrm{ArH}), 7.08$ (d, $1 \mathrm{H}, J=8.0 \mathrm{~Hz}, \operatorname{Ar} H), 7.01(\mathrm{~s}, 1 \mathrm{H}, \mathrm{Ar} H), 5.92$ (ddd, $1 \mathrm{H}, J=17.5,10.5,8.0 \mathrm{~Hz}, H-2), 5.35$ (dd, $1 \mathrm{H}, J$ $=10.5,1.0 \mathrm{~Hz}, H-1), 5.33-5.27(\mathrm{~m}, 2 \mathrm{H}, H-1, H-5), 4.66\left(\mathrm{~d}, 1 \mathrm{H}, J=11 \mathrm{~Hz}, \mathrm{OCH}_{2} \mathrm{Ph}\right), 4.58(\mathrm{~d}, 1 \mathrm{H}, J=11$ $\left.\mathrm{Hz}, \mathrm{OCH} \mathrm{H}_{2} \mathrm{Ph}\right), 4.55\left(\mathrm{~d}, 1 \mathrm{H}, J=12.0 \mathrm{~Hz}, \mathrm{OCH}_{2} \mathrm{Ph}\right), 4.52\left(\mathrm{~d}, 1 \mathrm{H}, J=12 \mathrm{~Hz}, \mathrm{OCH}_{2} \mathrm{Ph}\right), 4.48(\mathrm{~d}, 1 \mathrm{H}, J=12$ $\left.\mathrm{Hz}, \mathrm{OCH}_{2} \mathrm{Ph}\right), 4.20\left(\mathrm{~d}, 1 \mathrm{H}, J=12.0 \mathrm{~Hz}, \mathrm{OCH}_{2} \mathrm{Ph}\right), 3.88(\mathrm{dd}, 1 \mathrm{H}, J=8.0,5.0 \mathrm{~Hz}, H-3), 3.86-3.84(\mathrm{~m}, 2$ $\mathrm{H}, 2 \times H-6), 3.83(\mathrm{dd}, 1 \mathrm{H}, J=5.0,5.0 \mathrm{~Hz}, H-4), 3.55(\mathrm{~d}, 1 \mathrm{H}, J=15 \mathrm{~Hz}, H-7), 3.50(\mathrm{~d}, 1 \mathrm{H}, J=14.5 \mathrm{~Hz}$, H-7), 2.84-2.79 (m, $2 \mathrm{H}, 2 \times \mathrm{CH}), 2.34-2.27(\mathrm{~m}, 1 \mathrm{H}, \mathrm{CH}), 2.27-2.20(\mathrm{~m}, 1 \mathrm{H}, \mathrm{CH}), 1.95-1.89(\mathrm{~m}, 2 \mathrm{H}, 2 \mathrm{x}$ $\mathrm{CH}), 1.95-1.89(\mathrm{~m}, 2 \mathrm{H}, 2 \times \mathrm{CH}), 1.85-1.66(\mathrm{~m}, 3 \mathrm{H}, 3 \times \mathrm{CH}), 1.60-1.46(\mathrm{~m}, 2 \mathrm{H}, 2 \times \mathrm{CH}), 1.43-1.26(\mathrm{~m}, 6$ $\mathrm{H}, 6 \times \mathrm{CH}), 1.21-1.13(\mathrm{~m}, 1 \mathrm{H}, \mathrm{CH}), 0.77\left(\mathrm{~s}, 3 \mathrm{H}, \mathrm{CH}_{3}\right) ;{ }^{13} \mathrm{C} \mathrm{NMR}\left(125 \mathrm{MHz}, \mathrm{CDCl}_{3}\right) \delta 170.8,139.5$, $138.2,138.1,138.0,136.9,135.2,130.8,129.7,128.2$, 128.2, 128.1, 128.0, 127.9, 127.5, 127.5, 127.4, $126.4,125.5,119.2,80.9,80.5,74.9,73.0,73.0,70.3,68.0,53.5,44.2,41.1,40.9,40.4,38.7,38.7,29.4$, 27.9, 26.4, 25.1, 20.5, 17.5; HRMS (FAB) calcd for $\mathrm{C}_{47} \mathrm{H}_{54} \mathrm{O}_{5} \mathrm{Na}(M)^{+} 721.3863$, found 721.3849.

\section{C-Glycoside (6c)}

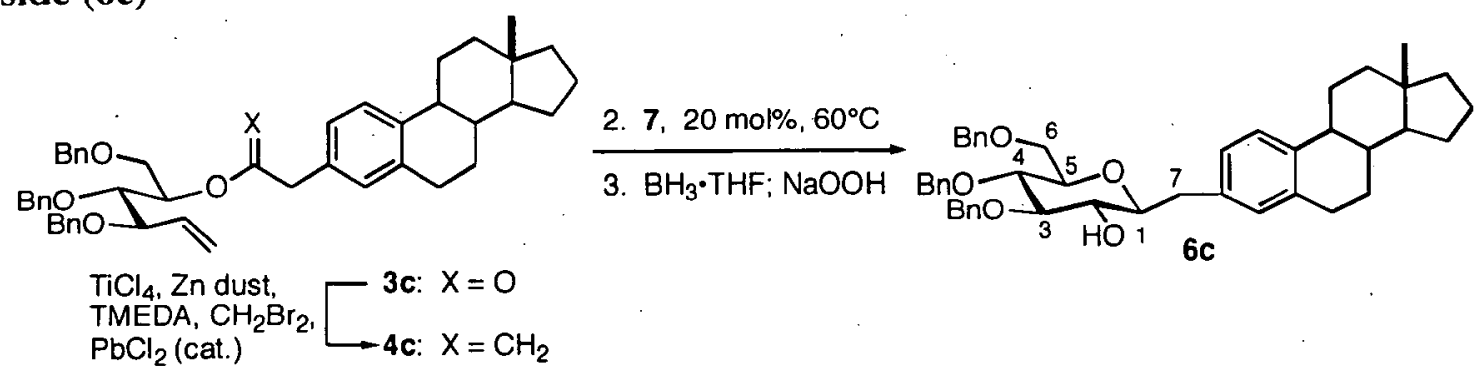

The general procedure for the three step protocol was followed using: Titanium tetrachloride $(1.19 \mathrm{~mL}, 2$ $\mathrm{M}$ in $\left.\mathrm{CH}_{2} \mathrm{Cl}_{2}, 2.38 \mathrm{mmol}\right)$, THF ( $\left.2 \mathrm{~mL}\right)$, TMEDA ( $\left.0.69 \mathrm{~mL}, 4.59 \mathrm{mmol}\right)$, zinc dust ( $\left.339 \mathrm{mg}, 5.18 \mathrm{mmol}\right)$, lead (II) chloride ( $4 \mathrm{mg}, 0.014 \mathrm{mmol})$, ester $3 \mathrm{c}$ ( $100 \mathrm{mg}, 0.14 \mathrm{mmol})$, dibromomethane ( $91 \mu \mathrm{L}, 1.3$ $\mathrm{mmol}$ ), catalyst 7 (30 mg, $0.158 \mathrm{mmol}, 25 \mathrm{~mol} \%)$, toluene ( $14 \mathrm{~mL}), \mathrm{BH}_{3} \cdot \mathrm{THF}(1.4 \mathrm{~mL}, 1 \mathrm{M}$ in THF, 1.4 $\mathrm{mmol}), \mathrm{NaOH}(10 \mathrm{~mL}, 1 \mathrm{M}, 10 \mathrm{mmol})$ and hydrogen peroxide $(10 \mathrm{~mL}, 30 \%$ in water, $0.05 \mathrm{~mol})$. After the usual work-up, flash chromatography of the residue over silica using $10 \rightarrow 20 \%$ EtOAc-hexanes gave 6c (52 mg, $52 \%$ over 3 steps) as a pure $\left(R_{f}=0.32\right.$, TLC, silica, $20 \%$ EtOAc-hexanes; ${ }^{1} \mathrm{H}$ NMR $(500 \mathrm{MHz}$, $\left.\mathrm{CDCl}_{3}\right)$ ) oil: $[\alpha]_{b}^{26}=+34.3\left(c=1.00, \mathrm{CHCl}_{3}\right)$; FT-IR (neat) $3441,3063,3030,2919,2865,1607,1497$, $1453,1359,1047,1027,908,831,731,697 \mathrm{~cm}^{-1} ;{ }^{1} \mathrm{H}$ NMR $\left(500 \mathrm{MHz}, \mathrm{CDCl}_{3}\right) \delta$ 7.41-7.30 (m, $\left.13 \mathrm{H}, \mathrm{Ph}\right)$, 7.29-7.25 (m, $2 \mathrm{H}, \mathrm{Ph}), 7.22(\mathrm{~d}, 1 \mathrm{H}, J=8.0 \mathrm{~Hz}, \mathrm{Ar} H), 7.12(\mathrm{~d}, 1 \mathrm{H}, J=8.0 \mathrm{~Hz}, \operatorname{Ar} H), 7.05(\mathrm{~s}, 1 \mathrm{H}, \operatorname{Ar} H)$, $5.00\left(\mathrm{~d}, 1 \mathrm{H}, J=12.4 \mathrm{~Hz}, \mathrm{OCH}_{2} \mathrm{Ph}\right), 4.84\left(\mathrm{~d}, 1 \mathrm{H}, J=10.4 \mathrm{~Hz}, \mathrm{OCH}_{2} \mathrm{Ph}\right), 4.77(\mathrm{~d}, 1 \mathrm{H}, J=11.6 \mathrm{~Hz}$, $\left.\mathrm{OCH}_{2} \mathrm{Ph}\right), 4.66\left(\mathrm{~d}, 1 \mathrm{H}, J=11.2 \mathrm{~Hz}, \mathrm{OCH}_{2} \mathrm{Ph}\right), 4.64\left(\mathrm{~d}, 1 \mathrm{H}, J=12.8 \mathrm{~Hz}, \mathrm{OCH}_{2} \mathrm{Ph}\right), 4.58(\mathrm{~d}, 1 \mathrm{H}, J=12$ $\mathrm{Hz}, \mathrm{OCH} \mathrm{Ph}_{2} \mathrm{Ph}, 3.78-3.74(\mathrm{~m}, 2 \mathrm{H}, 2 \times \mathrm{H}-6), 3.65(\mathrm{dd}, 1 \mathrm{H}, J=9.2,9.2 \mathrm{~Hz}, H-4), 3.53$ (dd, $1 \mathrm{H}, J=8.8,8.8$ $\mathrm{Hz}, H-3), 3.48-3.39$ (m, $2 \mathrm{H}, H-1, H-2, H-5), 3.10$ (dd, $1 \mathrm{H}, J=14.8,2.4 \mathrm{~Hz}, H-7), 2.90-2.75$ (m, $3 \mathrm{H}, H-$ 7, $2 \times \mathrm{CH}), 2.36-2.22(\mathrm{~m}, 2 \mathrm{H}, \mathrm{CH}, \mathrm{OH}), 1.95-1.88(\mathrm{~m}, 2 \mathrm{H}, 2 \times \mathrm{CH}), 1.84-1.78(\mathrm{~m}, 1 \mathrm{H}, \mathrm{CH}), 1.76-1.63$ $(\mathrm{m}, 2 \mathrm{H}, 2 \times \mathrm{CH}), 1.44-1.34(\mathrm{~m}, 3 \mathrm{H}, 3 \times \mathrm{CH}), 1.34-1.24(\mathrm{~m}, 3 \mathrm{H}, 3 \times \mathrm{CH}), 1.22-1.02(\mathrm{~m}, 1 \mathrm{H}, \mathrm{CH}), 0.78$ $\left(\mathrm{s}, 3 \mathrm{H}, \mathrm{CH}_{3}\right) ;{ }^{13} \mathrm{C}$ NMR $\left(125 \mathrm{MHz}, \mathrm{CDCl}_{3}\right) \delta 138.5,138.4,138.0,136.3,135.3,130.3,128.6,128.4$, $128.3,127.9,127.8,127.7,127.7,127.5,127.1,125.0,86.8,79.9,79.1,78.4,75.1,74.7,73.5,73.3,68.9$, 53.6, 44.3, 41.0, 40.5, 38.9, 38.8, 37.4, 29.6, 28.1, 26.5, 25.2, 20.5, 17.5; HRMS (FAB) calcd for $\mathrm{C}_{46} \mathrm{H}_{54} \mathrm{O}_{5} \mathrm{Na}(\mathrm{M})^{+} 709.3863$, found 709.3840 . 
(C) 2004 American Chemical Society, J. Org. Chem., Postema jo040254t Supporting Info Page 9 RCM-Based Synthesis of a Variety of $\beta-C$-Glycosides and their In Vitro Anti-Solid Tumor Activity

\section{ENTRY 4}

Benzyl 3-adamantyl-2-propenoate (10d)

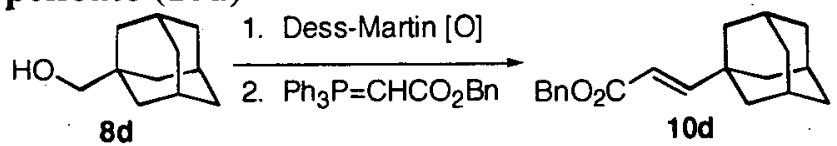

Alcohol 8d (1.1 g, $6.66 \mathrm{mmol})$ was dissolved in $\mathrm{CH}_{2} \mathrm{Cl}_{2}(150 \mathrm{~mL})$ and Dess-Martin periodane (4.22 $\mathrm{g}$, $9.98 \mathrm{mmol})$ was added portion-wise over $2 \mathrm{~min}$. After the usual work-up, the residue $9 \mathrm{~d}\left(\mathrm{R}_{\mathrm{f}}=0.25\right.$, TLC silica, 10\% EtOAc-hexanes; ' $\mathrm{H}$ NMR, $500 \mathrm{MHz}$ ) was dissolved in benzene and

(carbobenzyloxymethyl)triphenylphosphorane $(5.7 \mathrm{~g}, 13.99 \mathrm{mmol})$ was added in one portion. Stirring was continued for $8 \mathrm{~h}$, after which point no starting material remained (TLC silica, 10\%. EtOAc-hexanes). The solvent was removed in vacuo and the solid residue was subjected to flash chromatography over silica gel using $10 \rightarrow 15 \%$ EtOAc-hexanes to afford benzyl ester $10 \mathrm{~d}$ ( $1.73 \mathrm{~g}, 88 \%$ combined yield over 2 steps) as a $12: 1(E: Z)$ mixture of separable isomers. Major $(E)$ isomer: $\left(\mathrm{R}_{\mathrm{f}}=0.41\right.$, TLC, silica, $15 \%$ EtOAc-hexanes); FT-IR (neat) 2910, 2848, 2359, 2336, 1717, 1647, 1453, 1302, 1160, 989, 748, $696 \mathrm{~cm}$ ${ }^{1} ;{ }^{1} \mathrm{H}$ NMR $\left(500 \mathrm{MHz}, \mathrm{CDCl}_{3}\right) \delta 7.40-7.30(\mathrm{~m}, 5 \mathrm{H}, \mathrm{Ar} H), 5.77(\mathrm{~d}, 1 \mathrm{H}, J=13.0 \mathrm{~Hz}, \mathrm{C}=\mathrm{C}-H), 5.69(\mathrm{~d}, 1$ $\mathrm{H}, J=13.0 \mathrm{~Hz}, \mathrm{C}=\mathrm{C}-\mathrm{H}), 5.19\left(\mathrm{~s}, 2 \mathrm{H}, \mathrm{OCH}_{2} \mathrm{Ph}\right), 1.98(\mathrm{~s}, 3 \mathrm{H}, 3 \times \mathrm{CH}), 1.84\left(\mathrm{~s}, 6 \mathrm{H}, 3 \times \mathrm{CH}_{2}\right), 1.68(\mathrm{~s}, 6$ $\left.\mathrm{H}, 3 \times \mathrm{CH}_{2}\right) ;{ }^{13} \mathrm{C} \mathrm{NMR}\left(125 \mathrm{MHz} \mathrm{CDCl}_{3}\right) \delta 166.7,155.1,135.9,128.5,128.4,128.2,118.0,66.1,40.9$, 36.5, 28.4; HRMS (FAB) calcd for $\mathrm{C}_{20} \mathrm{H}_{24} \mathrm{O}_{2} \mathrm{Na}(\mathrm{M})^{+} 319.1668$, found 319.1669 .

\section{3-Adamantyl-2-propanoic acid (2d)}

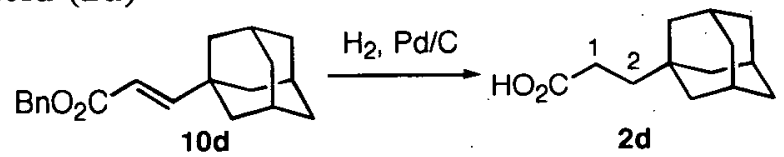

$\mathrm{Pd} / \mathrm{C}(10 \%, 20 \mathrm{mg})$ was added to an EtOAc $(10 \mathrm{~mL})$ solution of ester $10 d(800 \mathrm{mg}, 2.69 \mathrm{mmol})$ that was then shaken under an atmposphere of $\mathrm{H}_{2}(50 \mathrm{psi})$ for $12 \mathrm{~h}$. The solution was filtered through Celite using EtOAc as eluent $(100 \mathrm{~mL})$ and concentrated to a yellow oil. Flash chromatography over silica gel using $20 \rightarrow 35 \% \mathrm{Et}_{2} \mathrm{O}$-hexanes- $0.5 \% \mathrm{AcOH}$ gave adamantyl acid $2 \mathrm{~d}(500 \mathrm{mg}, 89 \%)$ as a pure $\left(\mathrm{R}_{\mathrm{f}}=0.41\right.$, TLC, silica, 30\% $\mathrm{Et}_{2} \mathrm{O}$-hexanes; ${ }^{1} \mathrm{H}$ NMR $\left(300 \mathrm{MHz}, \mathrm{CDCl}_{3}\right)$ ) white solid: m.p. $=134-135{ }^{\circ} \mathrm{C}$; FT-IR (neat) $2898,2846,2657,1696,1451,1421,1307,1103,940,735 \mathrm{~cm}^{-1}$; ${ }^{1} \mathrm{H}$ NMR $\left(300 \mathrm{MHz}, \mathrm{CDCl}_{3}\right) \delta 11.70$ (bs, $1 \mathrm{H}, \mathrm{COOH}), 2.35-2.27(\mathrm{~m}, 2 \mathrm{H}, 2 \times \mathrm{CH}), 1.95(\mathrm{~s}, 3 \mathrm{H}, 3 \times \mathrm{CH}), 1.70(\mathrm{~d}, 3 \mathrm{H}, J=11.7 \mathrm{~Hz}, 3 \times \mathrm{CH}), 1.61$ (d, $3 \mathrm{H}, J=11.4 \mathrm{~Hz}, 3 \times \mathrm{CH}), 1.48-1.39\left(\mathrm{~m}, 8 \mathrm{H}, 4 \times \mathrm{CH}_{2}\right) ;{ }^{13} \mathrm{C} \mathrm{NMR}\left(125 \mathrm{MHz}, \mathrm{CDCl}_{3}\right) \delta$ 181.6, 42.0, 38.6, 37.0, 31.9, 28.5, 27.9; HRMS (FAB) calcd for $\mathrm{C}_{13} \mathrm{H}_{20} \mathrm{O}_{2}(\mathrm{M})^{+} 208.1458$, found 208.1447.

\section{(3,4,6-tri-O-benzyl-1,2-dideoxy-D-arabino-hex-1-enyl) 3-adamantyl-2-propanoate (3d)}

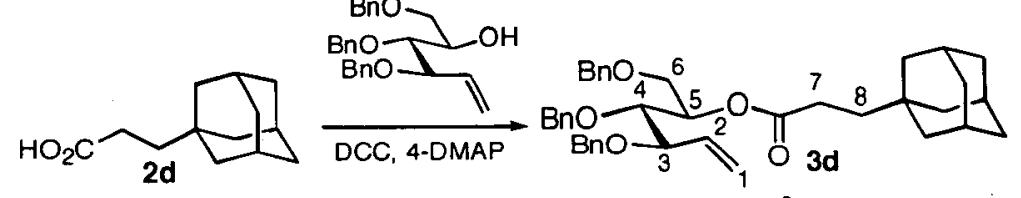

The general method for esterification was followed using: alcohol $1 \mathbf{a}^{5}$ (500 $\mathrm{mg}, 1.20 \mathrm{mmol}$ ), acid $2 \mathbf{d}$ (300 $\mathrm{mg}, 1.44 \mathrm{mmol}), \mathrm{DCC}(371 \mathrm{mg}, 1.80 \mathrm{mmol}), 4-\mathrm{DMAP}(73 \mathrm{mg}, 0.6 \mathrm{mmol})$ and $\mathrm{CH}_{2} \mathrm{Cl}_{2}(10 \mathrm{~mL})$. Flash chromatography of the residue over silica using $15 \rightarrow 25 \% \mathrm{Et}_{2} \mathrm{O}$-hexanes gave $3 \mathrm{~d}(678 \mathrm{mg}, 93 \%)$ as a pure $\left(\mathrm{R}_{\mathrm{f}}=0.41\right.$, TLC, silica, $25 \% \mathrm{Et}_{2} \mathrm{O}$-hexanes; ' $\left.\mathrm{H} \mathrm{NMR}\left(500 \mathrm{MHz}, \mathrm{CDCl}_{3}\right)\right)$ yellow oil: $[\alpha]_{\mathrm{D}}^{26}=-8.0(c=$ 1.00, $\mathrm{CHCl}_{3}$ ); FT-IR (neat) 3029, 2900, 2846, 2118, 1734, 1452, 1098, 1028, 734, $696 \mathrm{~cm}^{-1}$; ${ }^{1} \mathrm{H}$ NMR

\footnotetext{
${ }^{5}$ Freeman, F.; Robarge, K. D. Carbohydr. Res. 1987, 171, 1-11.
} 
(C) 2004 American Chemical Society, J. Org. Chem., Postema jo040254t Supporting Info Page 10

( $500 \mathrm{MHz}, \mathrm{CDCl}_{3}$ ) $87.34-7.26(\mathrm{~m}, 15 \mathrm{H}, \mathrm{Ar} H), 5.90$ (ddd, $\left.1 \mathrm{H}, J=17.5,10.0,7.5 \mathrm{~Hz}, \mathrm{H}-2\right), 5.32$ (dd, 1 $\mathrm{H}, J=10.0,1.5 \mathrm{~Hz}, H-1$ ), 5.28 (dd, $1 \mathrm{H}, J=18.5,1.0 \mathrm{~Hz}, H-1$ ), 5.21 (ddd, $1 \mathrm{H}, J=4.5,4.5,4.5 \mathrm{~Hz}, H-5$ ), $4.72\left(\mathrm{~d}, 1 \mathrm{H}, J=11 \mathrm{~Hz}, \mathrm{OCH}_{2} \mathrm{Ph}\right), 4.65\left(\mathrm{~d}, 1 \mathrm{H}, J=11.5 \mathrm{~Hz}, \mathrm{OCH}_{2} \mathrm{Ph}\right), 4.60(\mathrm{~d}, 1 \mathrm{H}, J=11.5 \mathrm{~Hz}$, $\left.\mathrm{OCH}_{2} \mathrm{Ph}\right), 4.49\left(\mathrm{~d}, 1 \mathrm{H}, J=12 \mathrm{~Hz}, \mathrm{OCH}_{2} \mathrm{Ph}\right), 4.46\left(\mathrm{~d}, 1 \mathrm{H}, J=12.5 \mathrm{~Hz}, \mathrm{OCH}_{2} \mathrm{Ph}\right), 4.32(\mathrm{~d}, 1 \mathrm{H}, J=12 \mathrm{~Hz}$, $\mathrm{OCH}_{2} \mathrm{Ph}$ ), 3.91 (dd, $\left.1 \mathrm{H}, J=8.0,5.0 \mathrm{~Hz}, H-3\right), 3.80$ (dd, $\left.1 \mathrm{H}, J=5.0,5.0 \mathrm{~Hz}, H-4\right), 3.79-3.77$ (m, $2 \mathrm{H}, 2 \mathrm{x}$ $H$-6), 2.18 (ddd, $1 \mathrm{H}, J=16,9.0,9.0 \mathrm{~Hz}, H-7$ ), 2.08 (ddd, $1 \mathrm{H}, J=16.5,9.5,9.5 \mathrm{~Hz}, H-7$ ), 1.95 (s, $3 \mathrm{H}, 3$ x CH), 1.70 (app d, $3 \mathrm{H}, J=12.0 \mathrm{~Hz}, 3 \times \mathrm{CH}), 1.61$ (app d, $3 \mathrm{H}, J=12.0 \mathrm{~Hz}, 3 \times \mathrm{CH}), 1.43$ (app d, $6 \mathrm{H}, J$ $\left.=2.0 \mathrm{~Hz}, 3 \times \mathrm{CH}_{2}\right), 1.35$ (app dd, $\left.2 \mathrm{H}, J=9.0,9.0 \mathrm{~Hz}, 2 \times \mathrm{H}-8\right) ;{ }^{13} \mathrm{C} \mathrm{NMR}\left(125 \mathrm{MHz}, \mathrm{CDCl}_{3}\right) \delta 174.0$, $138.6,138.4,138.3,135.5,128.6,128.5,128.5,128.3,128.3,127.9,127.9,127.8,119.6,80.7,80.6,75.0$, $73.0,72.3,70.3,68.1,42.0,38.7,37.0,31.8,28.5,28.1 ; \mathrm{HRMS}$ (FAB) calcd for $\mathrm{C}_{40} \mathrm{H}_{48} \mathrm{O}_{5} \mathrm{Na}(\mathrm{M})^{+}$ 631.3394 , found 631.3380 .

\section{2 '-(3; 4, 6-tri- $O$-benzyl- $\beta$-D-glucosyl)-1-ethyladamantane (6d):}

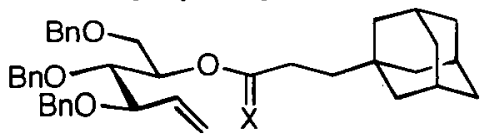

$\mathrm{TiCl}_{4}, \mathrm{Zn}$ dust, TMEDA, $\mathrm{CH}_{2} \mathrm{Br}_{2}$,

$\mathrm{PbCl}_{2}$ (cat.)
2. 7, $20 \mathrm{~mol} \%, 60^{\circ} \mathrm{C}$

3. $\mathrm{BH}_{3} \cdot \mathrm{THF} ; \mathrm{NaOOH}$

3d: $x=0$

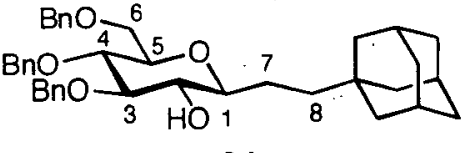

6d

The general procedure for the three step protocol was followed using: Titanium tetrachloride ( $1.4 \mathrm{~mL}, 2$ $\mathrm{M}$ in $\left.\mathrm{CH}_{2} \mathrm{Cl}_{2}, 2.79 \mathrm{mmol}\right)$, THF ( $\left.3 \mathrm{~mL}\right)$, TMEDA $(0.8 \mathrm{~mL}, 5.37 \mathrm{mmol})$, zinc dust (400 mg, $\left.6.07 \mathrm{mmol}\right)$, lead (II) chloride $(5 \mathrm{mg}, 0.016 \mathrm{mmol})$, ester $3 \mathrm{~d}(100 \mathrm{mg}, 0.16 \mathrm{mmol})$, dibromomethane $(107 \mu \mathrm{L}, 1.53$ $\mathrm{mmol})$, catalyst $7(28 \mathrm{mg}, 0.033 \mathrm{mmol}, 20 \mathrm{~mol} \%)$, toluene $(16 \mathrm{~mL}), \mathrm{BH}_{3} \cdot \mathrm{THF}(1.64 \mathrm{~mL}, 1 \mathrm{M}$ in THF, $1.64 \mathrm{mmol}), \mathrm{NaOH}(10 \mathrm{~mL}, 1 \mathrm{M}, 10 \mathrm{mmol})$ and hydrogen peroxide $(10 \mathrm{~mL}, 30 \%$ in water, $0.05 \mathrm{~mol})$. After the usual work-up, flash chromatography of the residue over silica using $10 \rightarrow 20 \%$ EtOAc-hexanes gave $6 \mathrm{~d}$ (57 mg, $0.096 \mathrm{mmol}, 60 \%$ over 3 steps) as a pure $\left(\mathrm{R}_{\mathrm{f}}=0.45, \mathrm{TLC}\right.$, silica, $35 \% \mathrm{Et}_{2} \mathrm{O}$-hexanes; ${ }^{1} \mathrm{H}$ NMR (500 MHz, $\left.\mathrm{CDCl}_{3}\right)$ ) oil: $[\alpha]_{D}^{26}=-7.3\left(c=1.00, \mathrm{CHCl}_{3}\right)$; FT-IR (neat) 3272, 2900, 2846, 1750, 1496, 1452, 1359, 1229, 1132, 1101, 1070, 1028, 734, $697 \mathrm{~cm}^{-1} ;{ }^{1} \mathrm{H}$ NMR $\left(500 \mathrm{MHz}, \mathrm{CDCl}_{3}\right) \delta 7.41-7.30(\mathrm{~m}, 13$ $\mathrm{H}, \mathrm{Ar} H), 7.27-7.24(\mathrm{~m}, 2 \mathrm{H}, \mathrm{Ar} H), 5.00\left(\mathrm{~d}, 1 \mathrm{H}, J=12 \mathrm{~Hz}, \mathrm{OCH}_{2} \mathrm{Ph}\right), 4.84(\mathrm{~d}, 1 \mathrm{H}, J=11.5 \mathrm{~Hz}$, $\left.\mathrm{OCH}_{2} \mathrm{Ph}\right), 4.76\left(\mathrm{~d}, 1 \mathrm{H}, J=12 \mathrm{~Hz}, \mathrm{OCH}_{2} \mathrm{Ph}\right), 4.68\left(\mathrm{~d}, 1 \mathrm{H}, J=12.5 \mathrm{~Hz}, \mathrm{OCH}_{2} \mathrm{Ph}\right), 4.65(\mathrm{~d}, 1 \mathrm{H}, J=10 \mathrm{~Hz}$, $\left.\mathrm{OCH}_{2} \mathrm{Ph}\right), 4.62\left(\mathrm{~d}, 1 \mathrm{H}, J=12.5 \mathrm{~Hz}, \mathrm{OCH}_{2} \mathrm{Ph}\right), 3.77$ (dd, $\left.1 \mathrm{H}, J=11.5,2.0 \mathrm{~Hz}, H-6\right), 3.73(\mathrm{dd}, 1 \mathrm{H}, J=$ $11.0,4.0 \mathrm{~Hz}, H-6), 3.66$ (dd, $1 \mathrm{H}, J=9.5,9.5 \mathrm{~Hz}, H-4), 3.48$ (dd, $1 \mathrm{H}, J=9.5,9.5 \mathrm{~Hz}, H-3$ ), 3.42 (ddd, 1 $\mathrm{H}, J=10,4.5,2.0 \mathrm{~Hz}, H-5$ ), 3.34 (ddd, $1 \mathrm{H}, J=9.5,9.5 \mathrm{~Hz}, H-2$ ), 3.12 (ddd, $1 \mathrm{H}, J=9.0,9.0,2.5 \mathrm{~Hz}, H$ 1), 2.15 (bs, $1 \mathrm{H}, \mathrm{OH}), 1.96$ (s, $3 \mathrm{H}, 3 \mathrm{x} \mathrm{CH}$ ), 1.86-1.78 (m, $1 \mathrm{H}, H-7$ ), 1.73 (app d, $3 \mathrm{H}, J=12 \mathrm{~Hz}, 3 \mathrm{x}$ $\mathrm{CH}), 1.65$ (d, $3 \mathrm{H}, J=11.5 \mathrm{~Hz}, 3 \times \mathrm{CH}), 1.51\left(\mathrm{~s}, 6 \mathrm{H}, 3 \times \mathrm{CH}_{2}\right), 1.49-1.39$ (m, $\left.2 \mathrm{H}, H-7, H-8\right), 1.09$ (ddd, 1 $\mathrm{H}, J=12.5,12.5,3.0 \mathrm{~Hz}, H-8) ;{ }^{13} \mathrm{C}$ NMR $\left(125 \mathrm{MHz} \mathrm{CDCl}_{3}\right) \delta 138.6,138.3,138.0,128.7,128.4,128.3$, $127.9,127.8,127.7,127.7,127.5,86.9,80.1,79.1,78.6,75.1,74.7,74.0,73.3,68.8,42.4,39.9,37.2$, 32.0, 28.7, 24.8; HRMS (FAB) calcd for $\mathrm{C}_{39} \mathrm{H}_{48} \mathrm{O}_{5} \mathrm{Na}(\mathrm{M})^{+} 619.3394$, found 619.3373.

\section{ENTRY 5}

Acid (2e)

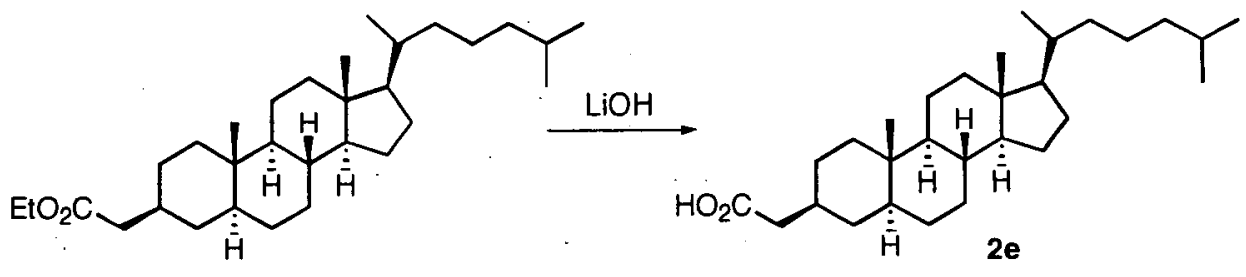


(C) 2004 American Chemical Society, J. Org. Chem., Postema jo040254t Supporting Info Page 11

$\mathrm{LiOH}(1.08 \mathrm{~g}, 25.8 \mathrm{mmol})$ was added to a cool $\left(0^{\circ} \mathrm{C}\right)$ solution of the steroidal ethyl ester ${ }^{6}(550 \mathrm{mg}, 1.29$ $\mathrm{mmol})$ in THF $(11 \mathrm{~mL})$. After 10 minutes, water $(11 \mathrm{~mL})$ was added and the resulting suspension was vigorously stirred at $0^{\circ} \mathrm{C}$ for $1 \mathrm{~h}$, allowed to warm to ambient temperature and then stirred overnight. The mixture was cooled to $0^{\circ} \mathrm{C}$ and acidified by the addition of $\mathrm{HCl}(10 \mathrm{~mL}, 2 \mathrm{M})$ until litmus red was obtained. The resulting solution was extracted with EtOAc $(5 \times 25 \mathrm{~mL})$, dried over $\mathrm{MgSO}_{4}$ and concentrated in vacuo. Flash chromatography of the residue over silica gel using $10 \rightarrow 15 \%$ EtOAchexanes- $0.5 \%$ AcOH gave the steroid-based acid $2 \mathrm{e}(500 \mathrm{mg}, 97 \%)$ as a pure $\left(\mathrm{R}_{\mathrm{f}}=0.33\right.$, TLC, silica, $15 \%$ EtOAc-hexanes $(0.5 \% \mathrm{AcOH}) ;{ }^{1} \mathrm{H}$ NMR $\left.\left(500 \mathrm{MHz}, \mathrm{CDCl}_{3}\right)\right)$ white solid: m.p. $=162-164^{\circ} \mathrm{C} ;[\alpha]_{\mathrm{D}}^{26}=+$ $19.4\left(c=1.00, \mathrm{CHCl}_{3}\right.$ ); FT-IR (neat) 3400-2800 (br), 2929, 2867, 2850, 1716, 1466, 1445, 1384, 1305, $1153,929 \mathrm{~cm}^{-1}$; ${ }^{1} \mathrm{H}$ NMR $\left(500 \mathrm{MHz}, \mathrm{CDCl}_{3}\right) \delta 2.24-2.20\left(\mathrm{~m}, 2 \mathrm{H}, \mathrm{CH}_{2}\right), 1.98-1.93(\mathrm{~m}, 1 \mathrm{H}, \mathrm{CH}), 1.84-$ $1.75(\mathrm{~m}, 2 \mathrm{H}, 2 \times \mathrm{CH}), 1.71-1.44(\mathrm{~m}, 6 \mathrm{H}, 6 \times \mathrm{CH}), 1.38-0.94(\mathrm{~m}, 23 \mathrm{H}, 23 \times \mathrm{CH}), 0.90(\mathrm{~d}, 3 \mathrm{H}, J=23 \mathrm{~Hz}$, $\mathrm{CH}-\mathrm{CH}_{3}$ ), 0.85 (app d, $6 \mathrm{H}, 2$ x CH-CH ), 0.76 (s, $3 \mathrm{H}, \mathrm{CH}_{3}$ ), 0.64 (s, $\left.3 \mathrm{H}, \mathrm{CH}_{3}\right)$; ${ }^{13} \mathrm{C} \mathrm{NMR}(125 \mathrm{MHz}$, $\left.\mathrm{CDCl}_{3}\right) \delta 179.3,56.5,56.3,54.5,46.4,42.6,41.8,40.1,39.5,38.3,36.2,35.8,35.7,35.5,35.2,35.1,32.1$, 29.7, 28.8, 28.5, 28.2, 28.0, 24.2, 23.8, 22.8, 22.6, 21.0, 18.7, 12.3, 12.1; HRMS (FAB) calcd for $\mathrm{C}_{29} \mathrm{H}_{50} \mathrm{O}_{2} \mathrm{Na}(\mathrm{M})^{+} 430.3811$, found 430.3815 .

Ester (3e)
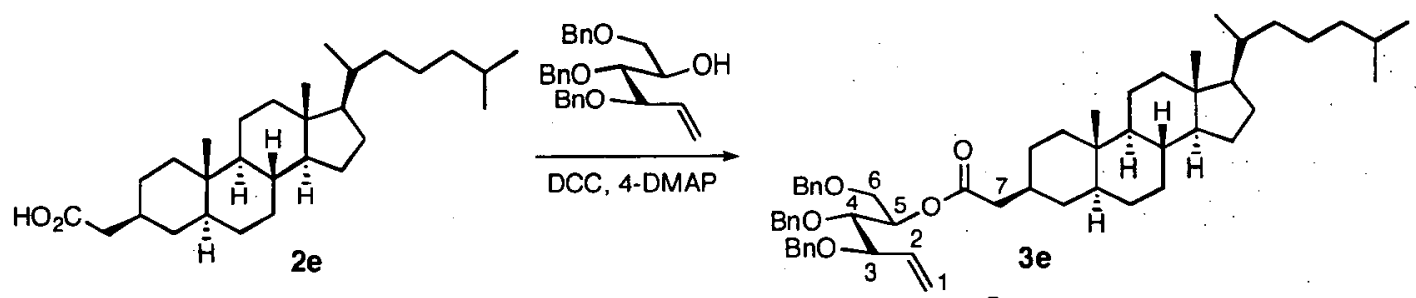

The general method for esterification was followed using: alcohol $1 \mathbf{a}^{7}(436 \mathrm{mg}, 1.05 \mathrm{mmol})$, acid $2 \mathrm{e}(500$ $\mathrm{mg}, 1.25 \mathrm{mmol}$ ), DCC (324 mg, $1.58 \mathrm{mmol}), 4-\mathrm{DMAP}(64 \mathrm{mg}, 0.53 \mathrm{mmol})$ and $\mathrm{CH}_{2} \mathrm{Cl}_{2}(8 \mathrm{~mL})$. Flash chromatography of the residue over silica gel using $10 \rightarrow 20 \%$ EtOAc-hexanes gave ester $3 \mathbf{e}(762 \mathrm{mg}$, $91 \%)$ as a pure $\left(\mathrm{R}_{\mathrm{f}}=0.61\right.$, TLC, silica, $20 \%$ EtOAc-hexanes; ${ }^{1} \mathrm{H} \mathrm{NMR}\left(500 \mathrm{MHz}, \mathrm{CDCl}_{3}\right)$ ) yellow oil: $[\alpha]_{D}^{26}=+8.4\left(c=1.00, \mathrm{CHCl}_{3}\right)$; FT-IR (neat) 2924, 2849, 1732, 1712, 1604, 1540, 1453, 1361, 1219, 1093; 1068, 734, $696 \mathrm{~cm}^{-1}$; ${ }^{1} \mathrm{H}$ NMR (500 MHz, $\mathrm{CDCl}_{3}$ ) $\delta 7.33-7.24$ (m, $15 \mathrm{H}, \mathrm{Ar} H$ ), 5.88 (ddd, $1 \mathrm{H}, J=17.5$, $10.0,8.0 \mathrm{~Hz}, H-2), 5.32$ (dd, $1 \mathrm{H}, \mathrm{J}=10.5,1.0, \mathrm{~Hz}, \mathrm{H}-1), 5.28$ (d, $1 \mathrm{H}, J=18 \mathrm{~Hz}, H-1$ ), 5.23 (ddd, $1 \mathrm{H}, J$ $=4.5,4.5,4.5 \mathrm{~Hz}, H-5), 4.71\left(\mathrm{~d}, 1 \mathrm{H}, J=11 \mathrm{~Hz}, \mathrm{OCH}_{2} \mathrm{Ph}\right), 4.64\left(\mathrm{~d}, 1 \mathrm{H}, J=11.5 \mathrm{~Hz}, \mathrm{OCH}_{2} \mathrm{Ph}\right), 4.57(\mathrm{~d}, 1$ $\left.\mathrm{H}, J=12 \mathrm{~Hz}, \mathrm{OCH}_{2} \mathrm{Ph}\right), 4.49-4.43\left(\mathrm{~m}, 2 \mathrm{H}, 2 \times \mathrm{OCH}_{2} \mathrm{Ph}\right), 4.31\left(\mathrm{~d}, 1 \mathrm{H}, J=11.5 \mathrm{~Hz}, \mathrm{OCH}_{2} \mathrm{Ph}\right), 3.91$ (dd, 1 $\mathrm{H}, J=8.0,5.0 \mathrm{~Hz}, H-3), 3.79(\mathrm{dd}, 1 \mathrm{H}, J=5.0,5.0 \mathrm{~Hz}, H-4), 3.78-3.76(\mathrm{~m}, 2 \mathrm{H}, 2 \mathrm{x} \mathrm{H-6}), 2.12-2.02(\mathrm{~m}, 2$ $\mathrm{H}, 2 \times H-7), 1.98-1.93(\mathrm{~m}, 1 \mathrm{H}, 1 \times \mathrm{CH}), 1.85-1.70(\mathrm{~m}, 2 \mathrm{H}, 2 \times \mathrm{CH}), 1.65-1.58(\mathrm{~m}, 2 \mathrm{H}, 2 \times \mathrm{CH}), 1.56-$ $1.50(\mathrm{~m}, 2 \mathrm{H}, 2 \times \mathrm{CH}), 1.50-1.43(\mathrm{~m}, 1 \mathrm{H}, \mathrm{CH}), 1.36-0.93(\mathrm{~m}, 20 \mathrm{H}, 20 \times \mathrm{CH}), 0.92-0.85(\mathrm{~m}, 3 \mathrm{H}, 3 \times$ $\mathrm{CH}), 0.90$ (d, $\left.3 \mathrm{H}, J=7.0 \mathrm{~Hz}, \mathrm{CHCH}_{3}\right), 0.87\left(\mathrm{~d}, 3 \mathrm{H}, J=6.5 \mathrm{~Hz}, \mathrm{CHCH}_{3}\right), 0.86(\mathrm{~d}, 3 \mathrm{H}, J=6.5 \mathrm{~Hz}$, $\left.\mathrm{CHCH}_{3}\right), 0.72\left(\mathrm{~s}, 3 \mathrm{H}, \mathrm{CH}_{3}\right), 0.64\left(\mathrm{~s}, 3 \mathrm{H}, \mathrm{CH}_{3}\right) ;{ }^{13} \mathrm{C} \mathrm{NMR}\left(125 \mathrm{MHz} \mathrm{CDCl}_{3}\right) \delta 172.2,138.1,138.1$, $138.1,135.2,128.3,128.3,128.2,128.0,127.6,127.6,127.5,119.4,81.0,80.9,75.0,73.0,72.3,70.4$, $68.1,56.6,56.3,54.5,46.3,42.6,42.2,40.0,39.5,38.2,36.2,35.8,35.5,35.3,32.0,28.8,28.5,28.2,28.0$, $24.2,23.8,22.8,22.6,21.0,18.7,12.3,12.1$; HRMS (FAB) calcd for $\mathrm{C}_{56} \mathrm{H}_{78} \mathrm{O}_{5} \mathrm{Na}(\mathrm{M})^{+} 853.5741$, found 853.5735 .

\footnotetext{
${ }^{6}$ Golebiewski, W. M.; Keyes, R. F,; Cushman, M. Bioorg. Med. Chem. 1996, 4, 1637-1648.

${ }^{7}$ Freeman, F.; Robarge, K. D. Carbohydr. Res. 1987, 171, 1-11.
} 
(C) 2004 American Chemical Society, J. Org. Chem., Postema jo040254t Supporting Info Page 12

\section{C-Glycoside (6e)}

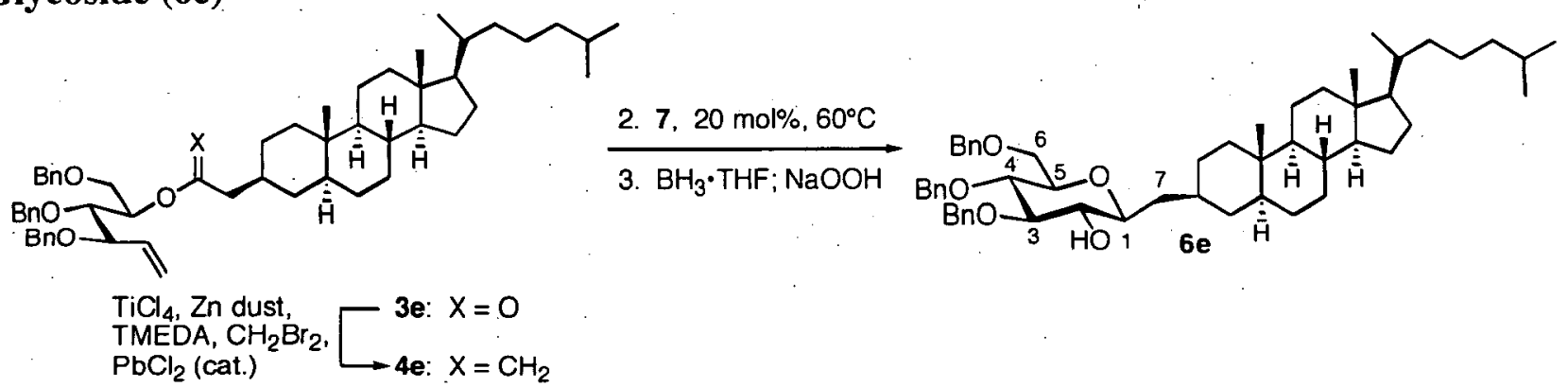

The general procedure for the three step protocol was followed using: Titanium tetrachloride $(1.02 \mathrm{~mL}, 2$ $\mathrm{M}$ in $\left.\mathrm{CH}_{2} \mathrm{Cl}_{2}, 2.04 \mathrm{mmol}\right)$, THF ( $\left.2 \mathrm{~mL}\right)$, TMEDA $(0.59 \mathrm{~mL}, 3.94 \mathrm{mmol})$, zinc dust (290 $\left.\mathrm{mg}, 4.44 \mathrm{mmol}\right)$, lead (II) chloride ( $3 \mathrm{mg}, 0.012 \mathrm{mmol})$, ester $3 \mathrm{e}(100 \mathrm{mg}, 0.12 \mathrm{mmol})$, dibromomethane ( $78 \mu \mathrm{L}, 1.12$ $\mathrm{mmol})$, catalyst 7 (20 mg, $0.024 \mathrm{mmol}, 20 \mathrm{~mol} \%)$, toluene $(12 \mathrm{~mL}), \mathrm{BH}_{3} \bullet \mathrm{THF}(1.2 \mathrm{~mL}, 1 \mathrm{M}$ in THF, 1.2 $\mathrm{mmol}), \mathrm{NaOH}(10 \mathrm{~mL}, 1 \mathrm{M}, 10 \mathrm{mmol})$ and hydrogen peroxide $(10 \mathrm{~mL}, 30 \%$ in water, $0.05 \mathrm{~mol})$. After the usual work-up, flash chromatography of the residue over silica using $10 \rightarrow 20 \%$ EtOAc-hexanes gave 6e $\left(47 \mathrm{mg}, 50 \%\right.$ over 3 steps) as a pure $\left(R_{\mathrm{f}}=0.42\right.$, TLC, silica, $20 \%$ EtOAc-hexanes; ${ }^{\prime} \mathrm{H} \mathrm{NMR}(500 \mathrm{MHz}$, $\left.\mathrm{CDCl}_{3}\right)$ ) white solid: m.p. $=42-43^{\circ} \mathrm{C} ;[\alpha]_{b}^{26}=+19.2\left(c=1.00, \mathrm{CHCl}_{3}\right) ; \mathrm{FT}-\mathrm{IR}$ (neat) 3489 (br), 2916, $2850,1454,1343,1162,1120,1063,902,816,699,668 \mathrm{~cm}^{-1} ;{ }^{1} \mathrm{H}$ NMR $\left(500 \mathrm{MHz}, \mathrm{CDCl}_{3}\right) \delta$ 7.38-7.27 $(\mathrm{m}, 13 \mathrm{H}, \mathrm{Ar} H), 7.24-7.21(\mathrm{~m}, 2 \mathrm{H}, \mathrm{Ar} H), 4.96\left(\mathrm{~d}, 1 \mathrm{H}, J=11.5 \mathrm{~Hz}, \mathrm{OCH}_{2} \mathrm{Ph}\right), 4.81(\mathrm{~d}, 1 \mathrm{H}, J=10.5 \mathrm{~Hz}$, $\left.\mathrm{OCH}_{2} \mathrm{Ph}\right), 4.72\left(\mathrm{~d}, 1 \mathrm{H}, J=11.5 \mathrm{~Hz}, \mathrm{OCH}_{2} \mathrm{Ph}\right), 4.64\left(\mathrm{~d}, 1 \mathrm{H}, J=12 \mathrm{~Hz}, \mathrm{OCH}_{2} \mathrm{Ph}\right), 4.61(\mathrm{~d}, 1 \mathrm{H}, J=11.5$ $\left.\mathrm{Hz}, \mathrm{OCH}_{2} \mathrm{Ph}\right), 4.57$ (d, $\left.1 \mathrm{H}, J=12 \mathrm{~Hz}, \mathrm{OCH}_{2} \mathrm{Ph}\right), 3.75$ (dd, $\left.1 \mathrm{H}, J=11.0,2.0 \mathrm{~Hz}, H-6\right), 3.69$ (dd, $1 \mathrm{H}, J=$ $11.5,5.0 \mathrm{~Hz}, H-6), 3.61$ (dd, $1 \mathrm{H}, J=9.5,9.5 \mathrm{~Hz}, H-4), 3.46$ (dd, $1 \mathrm{H}, J=9.0,9.5 \mathrm{~Hz}, H-3$ ), 3.40 (ddd, 1 $\mathrm{H}, J=10.0,4.0,2.0 \mathrm{~Hz}, H-5), 3.29-3.22(\mathrm{~m}, 2 \mathrm{H}, H-1, H-2), 1.99-1.93(\mathrm{~m}, 1 \mathrm{H}, \mathrm{CH}), 1.86-1.74(\mathrm{~m}, 2 \mathrm{H}, 2$ x CH), 1.69-1.46 (m, $8 \mathrm{H}, 8 \times \mathrm{CH}), 1.42-1.08(\mathrm{~m}, 18 \mathrm{H}, 18 \times \mathrm{CH}), 0.92-0.85(\mathrm{~m}, 4 \mathrm{H}, 4 \times \mathrm{CH}), 0.90(\mathrm{~d}, 3$ $\left.\mathrm{H}, J=8.0 \mathrm{~Hz}, \mathrm{CHCH}_{3}\right), 0.87$ (d, $\left.3 \mathrm{H}, J=9.0 \mathrm{~Hz}, \mathrm{CHCH}_{3}\right), 0.86$ (d, $\left.3 \mathrm{H}, J=8.0 \mathrm{~Hz}, \mathrm{CHCH}_{3}\right), 0.74$ (s, 3 , $\left.\mathrm{CH}_{3}\right), 0.64\left(\mathrm{~s}, 3 \mathrm{H}, \mathrm{CH}_{3}\right) ;{ }^{13} \mathrm{C} \mathrm{NMR}\left(100 \mathrm{MHz}, \mathrm{CDCl}_{3}\right) \delta 138.6,138.2,138.1,128.7,128.5,128.4,128.3$, $128.0,127.9,127.8,127.8,127.7,127.6,87.0,79.0,78.6,77.2,75.2,74.7,74.4,73.4,69.0,55.6,56.2$, $54.6,46.4,42.6,40.1,39.5,39.4,38.6,36.2,36.0,35.8,35.5,35.0,34.2,32.1,29.8,29.0,28.3,28.0,24.2$, 23.8, 22.8, 22.6, 21.0, 18.7, 12.3, 12.1; HRMS (FAB) calcd for $\mathrm{C}_{55} \mathrm{H}_{78} \mathrm{O}_{5} \mathrm{Na}(\mathrm{M})^{+} 841.5741$, found 841.5753 .

\section{ENTRY 6}

Ester (3f)

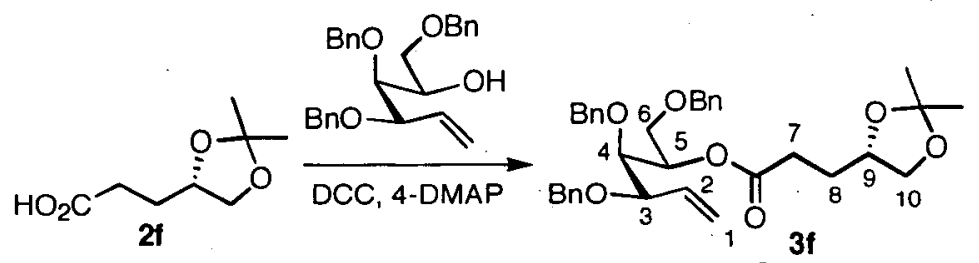

The general method for esterification was followed using: alcohol $1 \mathbf{b}^{8}(500 \mathrm{mg}, 1.19 \mathrm{mmol})$, acid $2 \mathbf{2 f}^{9}(250$ $\mathrm{mg}, 1.43 \mathrm{mmol})$, DCC (368 mg, $1.79 \mathrm{mmol}), 4-\mathrm{DMAP}(73 \mathrm{mg}, 0.60 \mathrm{mmol})$ and $\mathrm{CH}_{2} \mathrm{Cl}_{2}(5 \mathrm{~mL})$. Flash chromatography of the residue over silica using $20 \rightarrow 25 \% \mathrm{Et}_{2} \mathrm{O}$-hexanes gave $\mathbf{3 f}(662 \mathrm{mg}, 97 \%)$ as a pure $\left(\mathrm{R}_{\mathrm{f}}=0.30\right.$, TLC, silica, $20 \%$ EtOAc-hexanes; ${ }^{1} \mathrm{H}$ NMR $\left.\left(500 \mathrm{MHz}, \mathrm{CDCl}_{3}\right)\right)$ yellow oil: $[\alpha]^{26}=-16.9(c=$

8 Pearson, W. H., Hines, J. V. Tetrahedron Lett. 1991, 32, 5513-5516.

9 Siddiqui, M. A.; Marquez, V. E.; Driscoll, J. S. Barchi, Jr., J. J., Tetrahedron Lett. 1994, 35, 3263-3266. 
(C) 2004 American Chemical Society, J. Org. Chem., Postema jo040254t Supporting Info Page 13

Postema, Piper, Betts, Valeriote and Pietraszkewicz RCM-Based Synthesis of a Variety of $\beta$-C-Glycosides and their In Vitro Anti-Solid Tumor Activity

1.00, $\mathrm{CHCl}_{3}$ ); FT-IR (neat) 2916, 2868, 1736, 1496, 1454, 1369, 1249, 1209, 1154, 1062, 996, 932, 856, 736, $697 \mathrm{~cm}^{-1}$; 'H NMR (500 MHz, $\left.\mathrm{CDCl}_{3}\right) \delta 7.34-7.25(\mathrm{~m}, 15 \mathrm{H}, \mathrm{Ar} H), 5.92$ (ddd, $1 \mathrm{H}, J=17.5,10.5$, 8.0, $H$-2), 5.39 (ddd, $1 \mathrm{H}, J=5.5,5.5,5.5 \mathrm{~Hz}, H-5$ ), 5.38 (dd, $1 \mathrm{H}, J=10.5,1.5 \mathrm{~Hz}, H-1$ ), 5.31 (d, $1 \mathrm{H}, J$ $=17,1.5 \mathrm{~Hz}, \mathrm{H}-1), 4.74\left(\mathrm{~d}, 1 \mathrm{H}, J=11 \mathrm{~Hz}, \mathrm{OCH}_{2} \mathrm{Ph}\right), 4.56\left(\mathrm{~m}, 1 \mathrm{H}, \mathrm{J}=11 \mathrm{~Hz}, \mathrm{OCH}_{2} \mathrm{Ph}\right), 4.56(\mathrm{~m}, 1 \mathrm{H}, \mathrm{J}$ $\left.=11 \mathrm{~Hz}, \mathrm{OCH}_{2} \mathrm{Ph}\right), 4.48\left(\mathrm{~d}, 1 \mathrm{H}, J=12 \mathrm{~Hz}, \mathrm{OCH}_{2} \mathrm{Ph}\right), 4.43\left(\mathrm{~d}, 1 \mathrm{H}, J=11.5 \mathrm{~Hz}, \mathrm{OCH}_{2} \mathrm{Ph}\right), 4.30(\mathrm{~d}, 1 \mathrm{H}, J$ $=12 \mathrm{~Hz}, \mathrm{OCH} \mathrm{Ph}$ ), 4.05 (dddd, $1 \mathrm{H}, J=12.5,12.5,6.0,6.0 \mathrm{~Hz}, H-9$ ), 3.97 (dd, $1 \mathrm{H}, J=7.5,6.0 \mathrm{~Hz}, \mathrm{H}$ 10), 3.88 (dd, $1 \mathrm{H}, J=6.5,6.5 \mathrm{~Hz}, H-3), 3.83$ (dd, $1 \mathrm{H}, J=6.0,4.0 \mathrm{~Hz}, H-4), 3.59-3.53(\mathrm{~m}, 2 \mathrm{H}, 2 \times H$ 6), 3.49 (dd, $1 \mathrm{H}, J=7.0,7.0 \mathrm{~Hz}, H-10$ ), 2.42 (ddd, $1 \mathrm{H}, J=17.0,7.5,7.5 \mathrm{~Hz}, H-7$ ), 2.30 (ddd, $1 \mathrm{H}, J=$ $15.5,7.5,7.5 \mathrm{~Hz}, \mathrm{H}-7), 1.83-1.77(\mathrm{~m}, 2 \mathrm{H}, 2 \times \mathrm{H}-8), 1.39\left(\mathrm{~s}, 3 \mathrm{H}, \mathrm{C}\left(\mathrm{CH}_{3}\right)_{2}\right), 1.31\left(\mathrm{~s}, 3 \mathrm{H}, \mathrm{C}\left(\mathrm{CH}_{3}\right)_{2}\right) ;{ }^{13} \mathrm{C}$ NMR $\left(125 \mathrm{MHz}, \mathrm{CDCl}_{3}\right) \delta 172.5,138.2,138.1,137.8,135.5,128.3,128.3,128.2,128.0,127.9,127.7$, $127.7,127.6,127.5,108.9,80.0,79.2,74.8,74.5,73.1,71.6,70.2,69.0,68.0,30.6,28.8,27.2,25.9$; HRMS (FAB) calcd for $\mathrm{C}_{35} \mathrm{H}_{42} \mathrm{O}_{7} \mathrm{Na}(\mathrm{M})^{+} 597.2823$, found 597.2799.

\section{C-Glycoside (6f)}

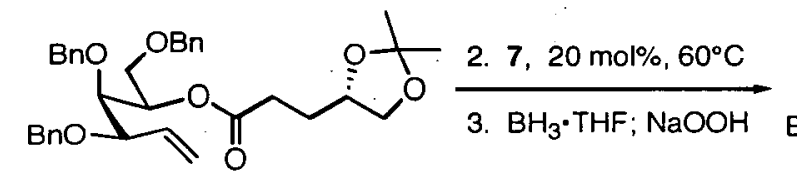

$\mathrm{TiCl}_{4}, \mathrm{Zn}$ dust,
$\mathrm{TMEDA}_{\mathrm{CMEH}} \mathrm{CH}_{2} \mathrm{Br}_{2} ; \longrightarrow$ 3f: $\mathrm{X}=0$
$\mathrm{PbCl}_{2}$ (cat.)

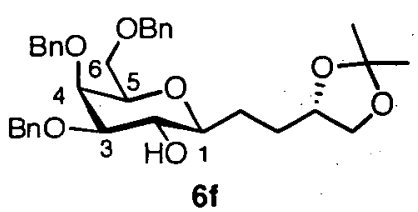

The general procedure for the three step protocol was followed using: Titanium tetrachloride ( $7.4 \mathrm{~mL}, 2$ $\mathrm{M}$ in $\left.\mathrm{CH}_{2} \mathrm{Cl}_{2}, 14.8 \mathrm{mmol}\right)$; THF (23 mL), TMEDA (4.3 mL, $\left.28.5 \mathrm{mmol}\right)$, zinc dust (2.1 g, $\left.32.2 \mathrm{mmol}\right)$, lead (II) chloride (24 mg, $0.09 \mathrm{mmol})$, ester $3 f(500 \mathrm{mg}, 0.87 \mathrm{mmol})$, dibromomethane $(0.57 \mathrm{~mL}, 8.09$ $\mathrm{mmol})$, catalyst 7 (148 mg, $0.17 \mathrm{mmol}, 20 \mathrm{~mol} \%)$, toluene (100 mL), BH ${ }_{3} \cdot \mathrm{THF}(8.7 \mathrm{~mL}, 1 \mathrm{M}$ in THF, 8.7 $\mathrm{mmol}), \mathrm{NaOH}(50 \mathrm{~mL}, 1 \mathrm{M}, 50 \mathrm{mmol})$ and hydrogen peroxide $(50 \mathrm{~mL}, 30 \%$ in water, $0.40 \mathrm{~mol})$. After the usual work-up, flash chromatography of the residue over silica using $20 \rightarrow 25 \%$ EtOAc-hexanes gave 6f $\left(278 \mathrm{mg}, 57 \%\right.$ over 3 steps) as a pure $\left(\mathrm{R}_{\mathrm{f}}=0.28\right.$, TLC, silica, 20\% EtOAc-hexanes; ${ }^{1} \mathrm{H}$ NMR (500 $\left.\mathrm{MHz}, \mathrm{CDCl}_{3}\right)$ ) oil: $[\alpha]_{\mathrm{b}}^{26}=-21.0\left(c=1.00, \mathrm{CHCl}_{3}\right)$; FT-IR (neat) $3420(\mathrm{br}), 2920,2865 ; 1454,1368,1210$, 1156, 1073, $911,848,734,697 \mathrm{~cm}^{-1} ;{ }^{1} \mathrm{H}$ NMR (500 MHz, $\left.\mathrm{CDCl}_{3}\right) \delta 7.38-7.25(\mathrm{~m}, 15 \mathrm{H}, \mathrm{ArH}), 4.85(\mathrm{~d}, 1$ $\left.\mathrm{H}, J=11.5 \mathrm{~Hz}, \mathrm{OCH}_{2} \mathrm{Ph}\right), 4.73\left(\mathrm{~d}, 1 \mathrm{H}, J=11.5 \mathrm{~Hz}, \mathrm{OCH}_{2} \mathrm{Ph}\right), 4.63\left(\mathrm{~d}, 1 \mathrm{H}, J=11.5 \mathrm{~Hz}, \mathrm{OCH}_{2} \mathrm{Ph}\right), 4.51$ $\left(\mathrm{d}, 1 \mathrm{H}, J=11.5 \mathrm{~Hz}, \mathrm{OCH} \mathrm{Ph}_{2}, 4.48\left(\mathrm{~d}, 1 \mathrm{H}, J=11.5 \mathrm{~Hz}, \mathrm{OCH}_{2} \mathrm{Ph}\right), 4.46\left(\mathrm{~d}, 1 \mathrm{H}, J=12.5 \mathrm{~Hz}, \mathrm{OCH} \mathrm{Ph}_{2}\right.\right.$, 4.11 (ddd, $1 \mathrm{H}, J=13.0,13.0,6.5,6.5 \mathrm{~Hz}, H-9), 4.04-4.00(\mathrm{~m}, 2 \mathrm{H}, H-10, H-4), 3.77$ (dd, $1 \mathrm{H}, J=9.5,9.5$ $\mathrm{Hz}, H-2), 3.61-3.55(\mathrm{~m}, 3 \mathrm{H}, 2 \times H-6, H-5), 3.53(\mathrm{dd}, 1 \mathrm{H}, J=7.5,7.5 \mathrm{~Hz}, H-10), 3.37$ (dd, $1 \mathrm{H}, J=9.5$, $3.0 \mathrm{~Hz}, H-3$ ), 3.17 (ddd, $1 \mathrm{H}, J=9.0,9.0,2.0 \mathrm{~Hz}, H-1$ ), 2.40 (bs, $1 \mathrm{H}, \mathrm{OH}$ ), 1.96-1.85 (m, $2 \mathrm{H}, H-7, H-8$ ), 1.67-1.53 (m, $2 \mathrm{H}, \mathrm{H}-7, \mathrm{H}-8), 1.40\left(\mathrm{~s}, 3 \mathrm{H}, \mathrm{C}\left(\mathrm{CH}_{3}\right)_{2}\right), 1.34\left(\mathrm{~s}, 3 \mathrm{H}, \mathrm{C}\left(\mathrm{CH}_{3}\right)_{2}\right) ;{ }^{13} \mathrm{C} \mathrm{NMR}\left(125 \mathrm{MHz}, \mathrm{CDCl}_{3}\right)$ $\delta 138.5,137.8,137.7,128.6,128.4,128.2,128.1,127.9,127.9,127.7,127.7,127.6,108.5,84.1,79.6$, $77.1,75.8,74.3,73.5,72.5,71.5,70.5,69.3,68.8,29.4,27.8,26.9,25.7$; HRMS (FAB) calcd for $\mathrm{C}_{34} \mathrm{H}_{42} \mathrm{O}_{7} \mathrm{Na}(\mathrm{M})^{+}$585.2823, found 585.2793.

Diol (15f)
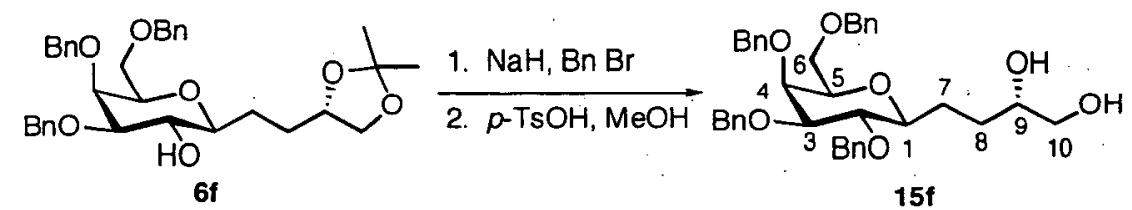

The usual method for benzylation was followed using: alcohol $6 \mathrm{f} \mathrm{(200} \mathrm{mg}, 0.36 \mathrm{mmol})$, $\mathrm{NaH}(50 \mathrm{mg}$, $1.24 \mathrm{mmol}), \mathrm{BnBr}(0.13 \mathrm{~mL}, 1.07 \mathrm{mmol})$ and $\mathrm{DMF}(2 \mathrm{~mL})$. Flash chromatography of the residue over 
(C) 2004 American Chemical Society, J. Org. Chem., Postema jo040254t Supporting Info Page 14

silica using $20 \rightarrow 25 \%$ EtOAc-hexanes gave the product $(207 \mathrm{mg}, 90 \%)$ as a pure $\left(R_{f}=0.35\right.$, TLC, silica, $20 \%$ EtOAc-hexanes; ${ }^{1} \mathrm{H}$ NMR (500 MHz, CDCl ${ }_{3}$ ), (HRMS (FAB) calcd for $\mathrm{C}_{41} \mathrm{H}_{48} \mathrm{O}_{7} \mathrm{Na}(\mathrm{M}){ }^{+} 675.3292$, found 675.3273)) clear oil that was taken to the next step without further characterization. $p$ - $\mathrm{TsOH}(5$ $\mathrm{mg}$ ) was added to a $\mathrm{MeOH}-\mathrm{CH}_{2} \mathrm{Cl}_{2}(1: 1,3 \mathrm{~mL})$ solution of the acetonide $(150 \mathrm{mg}, 0.23 \mathrm{mmol})$ and the resulting mixture was stirred for $12 \mathrm{~h}$ at ambient temperature. The reaction was quenched with $\mathrm{Et}_{3} \mathrm{~N}$ ( 1 $\mathrm{mL}$ ) and concentrated. Flash chromatography of the residue over silica using $2 \rightarrow 4 \% \mathrm{MeOH}-\mathrm{CH}_{2} \mathrm{Cl}_{2}$ gave $15 f\left(112 \mathrm{mg}, 80 \%, 72 \%\right.$ over 2 steps) as a pure $\left(\mathrm{R}_{\mathrm{f}}=0.40\right.$, TLC, silica, $5 \% \mathrm{MeOH}-\mathrm{CH}_{2} \mathrm{Cl}_{2} ;{ }^{1} \mathrm{H}$ NMR (500 $\left.\mathrm{MHz}, \mathrm{CDCl}_{3}\right)$ ) white solid: m.p. $=79-80^{\circ} \mathrm{C} ;[\alpha]_{\mathrm{b}}^{26}=+38.3\left(c=1.00, \mathrm{CHCl}_{3}\right) ; \mathrm{FT}-\mathrm{IR}$ (neat) $3386(\mathrm{br})$, $3063,3030,2915,2862,1497,1453,1359,1209,1063,909,735,697 \mathrm{~cm}^{-1} ;{ }^{1} \mathrm{H}$ NMR $\left(500 \mathrm{MHz}, \mathrm{CDCl}_{3}\right)$ S 7.40-7.28 (m, $20 \mathrm{H}, \mathrm{Ar} H) ; 4.97$ (d, $\left.1 \mathrm{H}, J=10.5 \mathrm{~Hz}, \mathrm{OCH}_{2} \mathrm{Ph}\right), 4.95\left(\mathrm{~d}, 1 \mathrm{H}, J=11.5 \mathrm{~Hz}, \mathrm{OCH}_{2} \mathrm{Ph}\right)$, $4.76\left(\mathrm{~d}, 1 \mathrm{H}, J=11.5 \mathrm{~Hz}, \mathrm{OCH}_{2} \mathrm{Ph}\right), 4.76\left(\mathrm{~d}, 1 \mathrm{H}, J=12 \mathrm{~Hz}, \mathrm{OCH}_{2} \mathrm{Ph}\right), 4.71(\mathrm{~d}, 1 \mathrm{H}, J=11.5 \mathrm{~Hz}$, $\left.\mathrm{OCH}_{2} \mathrm{Ph}\right) 4.63\left(\mathrm{~d}, 1 \mathrm{H}, J=11.5, \mathrm{OCH}_{2} \mathrm{Ph}\right), 4.48\left(\mathrm{~d}, 1 \mathrm{H}, J=11.5 \mathrm{~Hz}, \mathrm{OCH}_{2} \mathrm{Ph}\right), 4.41(\mathrm{~d}, 1 \mathrm{H}, J=11.5 \mathrm{~Hz}$, $\mathrm{OCH}_{2} \mathrm{Ph}$ ), 3.72-3.66 (m, $\left.2 \mathrm{H}, H-9, H-2\right)$, 3.63-3.48 (m, $5 \mathrm{H}, H-10,2$ x H-6, H-5, H-4), 3.44-3.34 (m, $2 \mathrm{H}$, $H-10, H-2$ ), 3.27 (ddd, $1 \mathrm{H}, J=9.0,9.0,1.5 \mathrm{~Hz}, H-1$ ), 2.16 (bs, $1 \mathrm{H}, \mathrm{OH}) 2.12-2.05$ (m, $1 \mathrm{H}, H-8$ ), 1.68 $1.56(\mathrm{~m}, 2 \mathrm{H}, H-7, H-8), 1.51$ (dddd, $1 \mathrm{H}, J=6.5,6.5,6.5,6.5 \mathrm{~Hz}, H-7) ;{ }^{13} \mathrm{C} \mathrm{NMR}\left(125 \mathrm{MHz}, \mathrm{CDCl}_{3}\right) \delta$ 138.4, 138.2, 138.1, 137.6, 128.5, 128.4, 128.2, 128.1, 128.0, 127.8, 127.8, 127.7, 127.5, 84.7, 80.3, 78.6, $77.1,75.5,74.4,73.6,73.5,72.3,72.0,69.4,66.8,30.6,28.3$; HRMS (FAB) calcd for $\mathrm{C}_{38} \mathrm{H}_{44} \mathrm{O}_{7} \mathrm{Na}(\mathrm{M})^{+}$ 635.2979 , found 635.2983 .

\section{ENTRY 7}

See experimental section in article.

\section{ENTRY $8^{10}$}

3-(2, 2-dimethyl-[1,3]dioxolan-4-yl)-propionic acid 2,3-bis-benzyloxy-1-benzyloxymethyl-pent-4enyl ester $(3 h)^{11}$

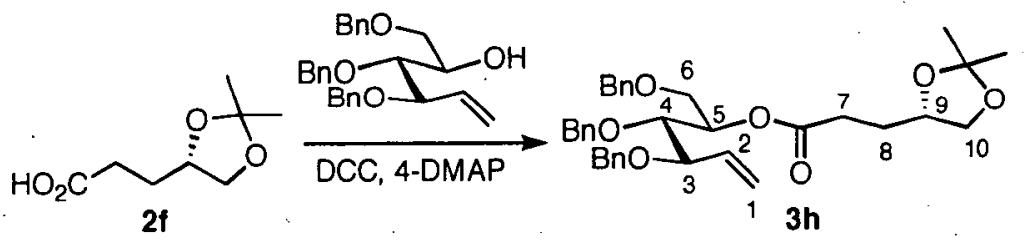

C-Glycoside $(6 \mathrm{~h})^{11}$
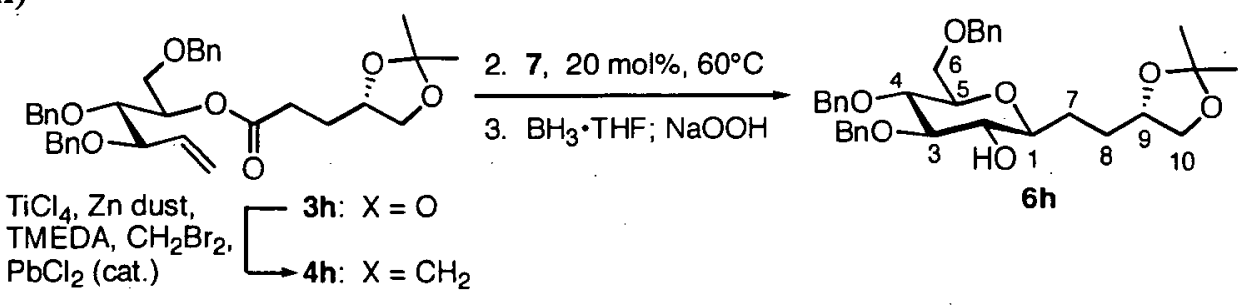

$\operatorname{Diol}(\mathbf{1 5 h})^{11}$

\footnotetext{
10 Note that the configuration of C-9 was drawn incorrectly in our original communication (Postema, M. H. D.; Piper, J. L. Org. Lett. 2003, 5, 1721-1723). The correct configuration is as depicted here.

${ }^{11}$ For full experimental and spectral data listing for this compound please see the supporting information corresponding to: Postema, M. H. D.; Piper, J. L. Org. Lett. 2003, 5, 1721-1723.
} 
(C) 2004 American Chemical Society, J. Org. Chem., Postema jo040254t Supporting Info Page 15 RCM-Based Synthesis of a Variety of $\beta$-C-Glycosides and their In Vitro Anti-Solid Tumor Activity

\section{ENTRY 9}
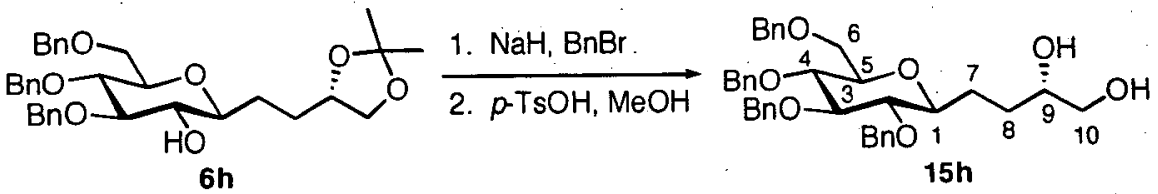

Ester (3i)

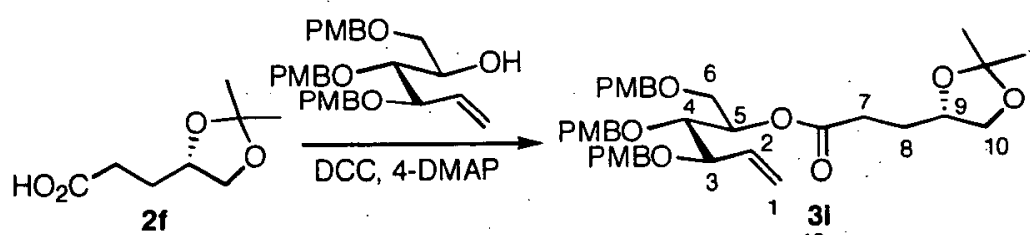

The general method for esterification was followed using: Alcohol $1 \mathrm{c}^{12}$ (580 $\left.\mathrm{mg}, 1.14 \mathrm{mmol}\right)$, acid $\mathbf{2 f}$ (240 mg, $1.37 \mathrm{mmol})$, DCC (353 mg, $1.71 \mathrm{mmol})$ and 4-DMAP (70 mg, 0.57. mmol) in $\mathrm{CH}_{2} \mathrm{Cl}_{2}(4 \mathrm{~mL})$. Flash chromatography of the residue over silica gel gave $3 \mathbf{i}(659 \mathrm{mg}, 87 \%)$ as a pure $\left(\mathrm{R}_{\mathrm{f}}=0.30, \mathrm{TLC}\right.$, silica $40 \%$ EtOAc-hexanes, ${ }^{1} \mathrm{H}$ NMR $\left.\left(500 \mathrm{MHz}, \mathrm{CDCl}_{3}\right)\right)$ pale yellow oil: $[\alpha]_{\mathrm{D}}^{26}=-12.0\left(c=1.00, \mathrm{CHCl}_{3}\right)$; FT-IR (neat) $2914,2868,1734,1612,1513,1464,1369,1247,1173,1071,1034,821 \mathrm{~cm}^{-1} ;{ }^{1} \mathrm{H}$ NMR $(500$

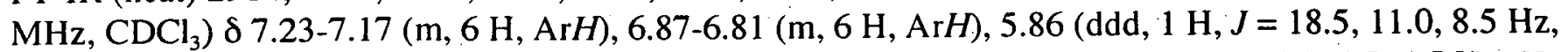
$H-2), 5.31(\mathrm{~d}, 1 \mathrm{H}, J=10 \mathrm{~Hz}, H-1), 5.26(\mathrm{~d}, 1 \mathrm{H}, J=17 \mathrm{~Hz}, H-1), 5.18$ (ddd, $1 \mathrm{H}, J=4.5,4.5,4.5 \mathrm{~Hz}, H$ 5), $4.60\left(\mathrm{~d}, 1 \mathrm{H}, J=11 \mathrm{~Hz}, \mathrm{OCH}_{2} \mathrm{Ar}\right), 4.54\left(\mathrm{~d}, 1 \mathrm{H}, J=11 \mathrm{~Hz}, \mathrm{OCH}_{2} \mathrm{Ar}\right), 4.52(\mathrm{~d}, 1 \mathrm{H}, J=11 \mathrm{~Hz}$, $\left.\mathrm{OCH}_{2} \mathrm{Ar}\right), 4.43\left(\mathrm{~d}, 1 \mathrm{H}, J=11.5 \mathrm{~Hz}, \mathrm{OCH}_{2} \mathrm{Ar}\right), 4.38\left(\mathrm{~d}, 1 \mathrm{H}, J=12 \mathrm{~Hz}, \mathrm{OCH}_{2} \mathrm{Ar}\right), 4.24(\mathrm{~d}, 1 \mathrm{H}, J=12 \mathrm{~Hz}$, $\mathrm{OCH}_{2} \mathrm{Ar}$ ), 4.65 (dddd, $1 \mathrm{H}, J=12.5,12.5,6.5,6.5 \mathrm{~Hz}, H-9$ ), 4.00 (dd, $1 \mathrm{H}, J=8.0,6.5 \mathrm{~Hz}, H-10$ ), 3.86 (dd, $1 \mathrm{H}, J=7.5,4.5 \mathrm{~Hz}, H-3), 3.79\left(\mathrm{~s}, 3 \mathrm{H}, \mathrm{OCH}_{3}\right), 3.78\left(\mathrm{~s}, 6 \mathrm{H}, 2 \times \mathrm{OCH}_{3}\right), 3.75-3.73(\mathrm{~m}, 1 \mathrm{H}, H-6)$, 3.73-3.71 (m, $2 \mathrm{H}, H-4, H-6), 3.49$ (dd, $1 \mathrm{H}, J=15.0,7.5 \mathrm{~Hz}, H-10), 2.37$ (ddd, $1 \mathrm{H}, J=16.0,6.5,6.5 \mathrm{~Hz}$, $H-7$ ), 2.18 (ddd, $1 \mathrm{H}, J=16.0,7.0,7.0 \mathrm{~Hz}, H-7), 1.81-1.74(\mathrm{~m}, 2 \mathrm{H}, 2 \times H-8), 1.40\left(\mathrm{~s}, 3 \mathrm{H}, \mathrm{CH}_{3}\right), 1.32$ (s, $\left.3 \mathrm{H}, \mathrm{CH}_{3}\right) ;{ }^{13} \mathrm{C}$ NMR $(125 \mathrm{MHz}) \delta 172.1,159.1,159.0,135.4,130.3,130.1,130.0,129.7,129.6,129.3$, $119.0,113.6,113.5,113.5,108.8,80.1,79.9,74.8,74.5,72.6,72.4,69.8,69.0,67.7,55.1,55.1,30.4$, $28.5,26.8,25.5$; HRMS(FAB): calcd for $\mathrm{C}_{38} \mathrm{H}_{48} \mathrm{O}_{10} \mathrm{Na}(\mathrm{M})^{+} 687.3140$, found 687.3163 .

\section{C-Glycoside (6i)}

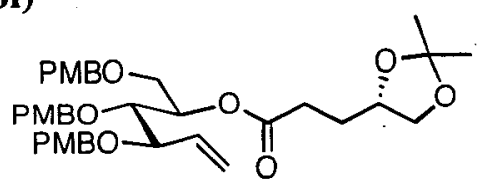

$\mathrm{TiCl}_{4}, \mathrm{Zn}$ dust, TMEDA, $\mathrm{CH}_{2} \mathrm{Br}_{2}$

$\mathrm{PbCl}_{2}$ (cat.)

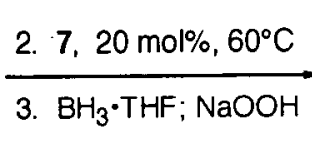

,

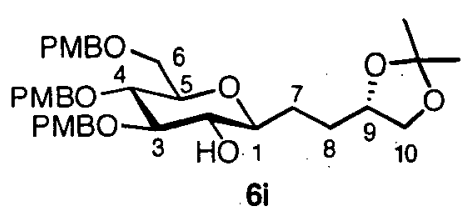

$6 \mathbf{i}$

The general procedure for the three step protocol was followed using: Titanium tetrachloride ( $3.2 \mathrm{~mL}, 2$ $\mathrm{M}$ in $\left.\mathrm{CH}_{2} \mathrm{Cl}_{2}, 6.39 \mathrm{mmol}\right)$, THF (10 mL), TMEDA (1.88 mL, $\left.12.46 \mathrm{mmol}\right)$, zinc dust $(0.92 \mathrm{~g}, 14.06$ $\mathrm{mmol}$ ), lead (II) chloride (11 mg, $0.04 \mathrm{mmol})$, ester $3 \mathrm{i}(250 \mathrm{mg}, 0.38 \mathrm{mmol})$, dibromomethane $(0.25 \mathrm{~mL}$, $3.53 \mathrm{mmol})$, catalyst $7(80 \mathrm{mg}, 0.094 \mathrm{mmol}, 25 \mathrm{~mol} \%)$, toluene $(37 \mathrm{~mL}), \mathrm{BH}_{3} \bullet \mathrm{THF}(3.8 \mathrm{~mL}, 1 \mathrm{M}$ in THF, $3.8 \mathrm{mmol}), \mathrm{NaOH}(20 \mathrm{~mL}, 1 \mathrm{M}, 20 \mathrm{mmol})$ and hydrogen peroxide $(20 \mathrm{~mL}, 30 \%$ in water, $0.16 \mathrm{~mol})$. After the usual work-up, flash chromatography of the residue over silica using $30 \rightarrow 40 \%$ EtOAc-hexanes gave $6 \mathbf{i}$ (124 mg, 50\% over 3 steps) as a pure $\left(R_{f}=0.26\right.$, TLC, silica, 40\% EtOAc-hexanes; ${ }^{1} \mathrm{H}$ NMR (500 $\left.\mathrm{MHz}, \mathrm{CDCl}_{3}\right)$ ) white solid: m.p. $=81-82^{\circ} \mathrm{C} ;\left[\alpha b^{26}==+32.7\left(c=1.00, \mathrm{CHCl}_{3}\right) ;\right.$ FT-IR (neat) 3478,2933 , $2866,1612,1513,1464,1368,1302,1247,1094,1035,821 \mathrm{~cm}^{-1} ;{ }^{1} \mathrm{H}$ NMR $\left(500 \mathrm{MHz}, \mathrm{CDCl}_{3}\right) \delta 7.30-$

\footnotetext{
12 Postema, M. H. D.; Piper, J. L. Org. Lett. 2003, 5, 1721-1723.
} 
(C) 2004 American Chemical Society, J. Org. Chem., Postema jo040254t Supporting Info Page 16

7.24 (m, $4 \mathrm{H}, \mathrm{Ar} H), 7.13-7.08(\mathrm{~m}, 2 \mathrm{H}, \mathrm{ArH}), 6.92-6.81(\mathrm{~m}, 6 \mathrm{H}, \mathrm{ArH}), 4.90\left(\mathrm{~d}, 1 \mathrm{H}, J=12 \mathrm{~Hz}, \mathrm{OCH} \mathrm{Ar}_{2}\right.$, $4.71\left(\mathrm{~d}, 1 \mathrm{H}, J=10.5 \mathrm{~Hz}, \mathrm{OCH}_{2} \mathrm{Ar}\right), 4.65\left(\mathrm{~d}, 1 \mathrm{H}, J=11 \mathrm{~Hz}, \mathrm{OCH}_{2} \mathrm{Ar}\right), 4.57$ (d, $1 \mathrm{H}, J=11.5 \mathrm{~Hz}$, $\mathrm{OCH}_{2} \mathrm{Ar}$ ), 4.49 (d, $\left.1 \mathrm{H}, J=10 \mathrm{~Hz}, \mathrm{OCH}_{2} \mathrm{Ar}\right), 4.48$ (d, $1 \mathrm{H}, J=11.5 \mathrm{~Hz}, \mathrm{OCH}_{2} \mathrm{Ar}$ ), 4.11 (dddd, $1 \mathrm{H}, J=$ $12.5,12.5,6.5,6.5 \mathrm{~Hz}, H-9), 4.03$ (dd, $1 \mathrm{H}, J=7.5,6.0 \mathrm{~Hz}, H-10), 3.80(\mathrm{~s}, 6 \mathrm{H}, 2$ x OCH ), 3.79 (s, $3 \mathrm{H}$, $\mathrm{OCH}_{3}$ ); 3.68-3.62 (m, $2 \mathrm{H}, 2$ x H-6), 3.55 (dd, $\left.1 \mathrm{H}, J=9.5,9.5 \mathrm{~Hz}, H-4\right), 3.52$ (dd, $1 \mathrm{H}, J=7.5,7.5 \mathrm{~Hz}$, $H-10), 3.41$ (dd, $1 \mathrm{H}, J=9.0,9.0 \mathrm{~Hz}, H-3), 3.36$ (ddd, $1 \mathrm{H}, J=9.5,3.0,3.0 \mathrm{~Hz}, H-5), 3.27(\mathrm{dd}, 1 \mathrm{H}, J=$ 9.0, 9.0 Hz, $H-2$ ), 3.14 (ddd, $1 \mathrm{H}, J=8.5,8.5,1.5 \mathrm{~Hz}, H-1$ ), 2.16 (br s, $1 \mathrm{H}, \mathrm{O} H$ ), 1.95-1.86 (m, $1 \mathrm{H}, H-8$ ), 1.86-1.78 (m, $1 \mathrm{H}, H-7), 1.62-1.53(\mathrm{~m}, 2 \mathrm{H}, \mathrm{H}-7, H-8), 1.40\left(\mathrm{~s}, 3 \mathrm{H}, \mathrm{CH}_{3}\right), 1.34\left(\mathrm{~s}, 3 \mathrm{H}, \mathrm{CH}_{3}\right) ;{ }^{13} \mathrm{C}$ NMR $\left(125 \mathrm{MHz}_{1} \mathrm{CDCl}_{3}\right) \delta 159.3,159.2,159.2,130.6,130.1,130.1,129.6,129.5,114.1,113.8,113.7,108.6$, $86.3,79.0,78.1,75.9,74.7,74.4,73.7,73.1,69.4 ; 68.4,55.2,55.2,29.3,27.7,26.9,25.7$; HRMS (FAB) calcd for $\mathrm{C}_{37} \mathrm{H}_{48} \mathrm{O}_{10} \mathrm{Na}(\mathrm{M})^{+} 675.3140$, found 675.3145 .

\section{Diol (15i)}
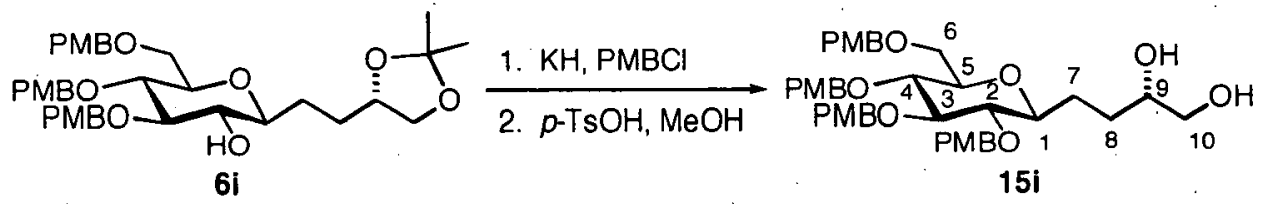

To a stirred solution of $\mathrm{KH}(50 \mathrm{mg}, 30 \%$ dispersion in mineral oil, $0.38 \mathrm{mmol})$ and $\mathrm{DMF}(3 \mathrm{~mL})$ at $0{ }^{\circ} \mathrm{C}$ was added alcohol $6 \mathbf{i}(70 \mathrm{mg}, 0.11 \mathrm{mmol})$ dropwise over 5 minutes. After 10 minutes, $\mathrm{PMBCl}(45 \mu \mathrm{L}$, $0.33 \mathrm{mmol}$ ) was added dropwise followed by tetrabutylammonium iodide ( $10 \mathrm{mg}, 0.027 \mathrm{mmol})$. Stirring was continued for 30 minutes at $0^{\circ} \mathrm{C}$ and then for $5 \mathrm{~h}$ at ambient temperature. The solution was quenched by dropwise addition of saturated $\mathrm{NH}_{4} \mathrm{Cl}(10 \mathrm{~mL})$ and extracted with EtOAc $(3 \times 10 \mathrm{~mL})$. The combined organic layers were washed with brine $(1 \times 10 \mathrm{~mL})$, dried over $\mathrm{MgSO}_{4}$, filtered, and concentrated. Flash chromatography of the residue over silica using $25 \rightarrow 30 \%$ EtOAc-hexanes gave the product (68 $\mathrm{mg}, 80 \%)$ as a pure $\left(R_{\mathrm{f}}=0.25\right.$, TLC, silica, $30 \%$ EtOAc-hexanes; ${ }^{1} \mathrm{H}$ NMR (500 MHz, $\mathrm{CDCl}_{3}$; HRMS (FAB) calcd for $\mathrm{C}_{45} \mathrm{H}_{56} \mathrm{O}_{11} \mathrm{Na}(\mathrm{M})^{+} 795.3715$, found 795.3682) oil which was taken to the next step without any further characterization. The usual procedure for acetonide deprotection was employed using: $\mathrm{CH}_{2} \mathrm{Cl}_{2}(2 \mathrm{~mL})$, $\mathrm{MeOH}(2 \mathrm{~mL}), p$-TsOH $(5 \mathrm{mg}, 0.026 \mathrm{mmol})$ and the acetonide sugar $(50 \mathrm{mg}, 0.065 \mathrm{mmol})$. After the usual work-up, flash chromatography of the residue over silica using $2 \rightarrow 4 \% \mathrm{CH}_{2} \mathrm{Cl}_{2}-\mathrm{MeOH}$ gave $15 \mathbf{i}$ (43 $\mathrm{mg}, 90 \%)$ as a pure $\left(\mathrm{R}_{\mathrm{f}}=0.20\right.$, TLC, silica, $\left.5 \% \mathrm{CH}_{2} \mathrm{Cl}_{2}-\mathrm{MeOH} ;{ }^{1} \mathrm{H} \mathrm{NMR}\left(500 \mathrm{MHz}, \mathrm{CDCl}_{3}\right)\right)$ white solid: m. p. $=104-105^{\circ} \mathrm{C} ;[\alpha]_{D}^{26}=+2.5\left(c=1.00, \mathrm{CHCl}_{3}\right)$; FT-IR (neat) 3383, 3000, 2904, 2854, 1613, 1514, 1466, 1301, 1252, 1173, 1063, 1033,820, $760 \mathrm{~cm}^{-1} ;{ }^{1} \mathrm{H}$ NMR $\left(500 \mathrm{MHz}, \mathrm{CDCl}_{3}\right) \delta 7.27$ (app dd, $2 \mathrm{H}, J=$ $9.0 \mathrm{~Hz}, \mathrm{Ar} H$ ), 7.22 (app d, $2 \mathrm{H}, J=8.5 \mathrm{~Hz}, \mathrm{ArH}), 6.88$ (app dd, $3 \mathrm{H}, J=2.5 \mathrm{~Hz}, \mathrm{ArH}$ ), 6.86 (app dd, $3 \mathrm{H}$, $J=3.0 \mathrm{~Hz}, \mathrm{Ar} H), 6.82($ app d, $2 \mathrm{H}, J=9.0 \mathrm{~Hz}, \mathrm{ArH}), 4.87-4.83\left(\mathrm{~m}, 2 \mathrm{H}, 2 \times \mathrm{OCH}_{2} \mathrm{Ar}\right), 4.82(\mathrm{~d}, 1 \mathrm{H}, J=$ $\left.11 \mathrm{~Hz}, \mathrm{OCH}_{2} \mathrm{Ar}\right), 4.74\left(\mathrm{~d}, 1 \mathrm{H}, J=10.5 \mathrm{~Hz}, \mathrm{OCH} \mathrm{Ar}_{2}, 4.56\left(\mathrm{~d}, 1 \mathrm{H}, J=11 \mathrm{~Hz}, \mathrm{OCH} \mathrm{H}_{2} \mathrm{Ar}\right), 4.51(\mathrm{~d}, 1 \mathrm{H}, J=\right.$ $11.5 \mathrm{~Hz}, \mathrm{OCH}_{2} \mathrm{Ar}$ ), 4.44 (d, $\left.1 \mathrm{H}, J=12 \mathrm{~Hz}, \mathrm{OCH} \mathrm{H}_{2} \mathrm{Ar}\right), 4.43\left(\mathrm{~d}, 1 \mathrm{H}, J=11.5 \mathrm{~Hz}, \mathrm{OCH}_{2} \mathrm{Ar}\right), 4.02(\mathrm{dd}, 1 \mathrm{H}$, $J=7.5,6.0 \mathrm{~Hz}, \mathrm{H}-10), 3.80\left(\mathrm{~s}, 3 \mathrm{H}, \mathrm{OCH}_{3}\right), 3.80\left(\mathrm{~s}, 3 \mathrm{H}, \mathrm{OCH}_{3}\right), 3.79\left(\mathrm{~s}, 3 \mathrm{H}, \mathrm{OCH}_{3}\right), 3.79(\mathrm{~s}, 3 \mathrm{H}$, $\mathrm{OCH}_{3}$ ), 3.66 (dddd, $\left.1 \mathrm{H}, J=7.0,7.0,3.0,3.0 \mathrm{~Hz}, H-9\right), 3.65-3.61(\mathrm{~m}, 2 \mathrm{H}, 2 \times H-6), 3.53$ (dd, $1 \mathrm{H}, J=11$, 3.5, Hz, $H-10), 3.34$ (ddd, $1 \mathrm{H}, J=10,4.5,2.0 \mathrm{~Hz}, H-5), 3.46-3.41(\mathrm{~m}, 2 \mathrm{H}, 2 \mathrm{X} \mathrm{OCH}), 3.26(J=9.5,9.5$, $2.0 \mathrm{~Hz}, H-1), 3.24-3.18(\mathrm{dd}, 1 \mathrm{H}, J=9.5,9.5 \mathrm{~Hz}, \mathrm{OCH}), 2.06-1.97(\mathrm{~m}, 1 \mathrm{H}, H-7), 1.64-1.56(\mathrm{~m}, 1 \mathrm{H}, H$ 8), 1.55-1.45 (m, $2 \mathrm{H}, \mathrm{H}-7, H-8) ;{ }^{13} \mathrm{C}$ NMR $\left(125 \mathrm{MHz}, \mathrm{CDCl}_{3}\right) \delta 159.3,159.3,159.3,159.2,130.7,130.1$, $130.0,129.7,129.7,129.7,129.6,129.3,113.8,113.8,113.7,86.8,81.6,79.7,78.4,78.3,75.2,74.9,74.6$, 73.0, 72.2, 68.8, 66.8, 55.3, 55.2, 55.2, 30.4, 28.4; HRMS (FAB) calcd for $\mathrm{C}_{42} \mathrm{H}_{52} \mathrm{O}_{11} \mathrm{Na}(\mathrm{M})^{+} 755.3402$, found 755.3396 .

\section{ENTRY 10}


(C) 2004 American Chemical Society, J. Org. Chem., Postema jo040254t Supporting Info Page 17 RCM-Based Synthesis of a Variety of $\beta-C$-Glycosides and their In Vitro Anti-Solid Tumor Activity

\section{C-Glycoside (6j)}

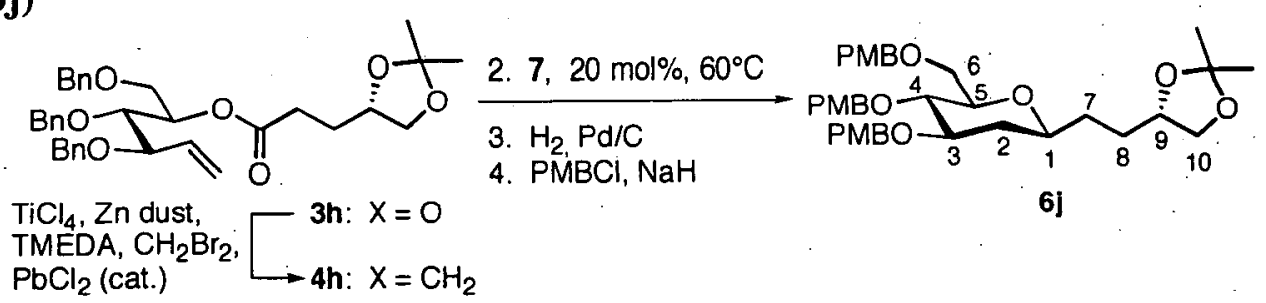

The two-step protocol was followed using:

(a) Titanium tetrachloride $\left(7.39 \mathrm{~mL}, 2 \mathrm{M}\right.$ in $\left.\mathrm{CH}_{2} \mathrm{Cl}_{2}, 14.8 \mathrm{mmol}\right)$, THF (23 mL), TMEDA (4.3 mL, $28.5 \mathrm{mmol}$ ), zinc dust $(2.1 \mathrm{~g}, 32.2 \mathrm{mmol}$ ), lead (II) chloride (25 mg, $0.09 \mathrm{mmol})$, ester $3 \mathrm{~h}(500 \mathrm{mg}, 0.87$ $\mathrm{mmol})$, dibromomethane $(0.57 \mathrm{~mL}, 8.1 \mathrm{mmol})$ and THF $(10 \mathrm{~mL})$.

(b) Ruthenium-based metathesis catalyst $7(221 \mathrm{mg}, 0.26 \mathrm{mmol}, 30 \mathrm{~mol} \%)$, added in 5 portions over 2 hours to a dry and degassed toluene $(87 \mathrm{~mL})$ solution of the intermediate acyclic enol ether. After the usual work-up the solution was concentrated.

The material was carried an additional two steps:

(c) The crude glycal was re-dissolved in a solution of EtOAc-EtOH (12 mL, 5:1) and shaken under at atmosphere of hydrogen gas $(55 \mathrm{psi})$ for $6 \mathrm{~h}$. The mixture was filtered through Celite and rinsed with with $200 \mathrm{~mL}$ EtOAc-MeOH (5:2), concentrated in vacuo and taken up in DMF (15 mL).

(d) The product of the previous sequence was added dropwise to a stirring solution of rinsed ( $2 \times 10$ $\mathrm{mL}$ THF) $\mathrm{NaH}(44 \mathrm{mg}, 1.84 \mathrm{mmol})$ in DMF $(5 \mathrm{~mL})$ at $0^{\circ} \mathrm{C}$. The reaction was stirred for 15 minutes at which point PMBCl $(0.28 \mathrm{~mL}, 2.1 \mathrm{mmol})$ was added, along with a catalytic amount of TBAI $(5 \mathrm{mg})$. After $1 \mathrm{~h}$, the solution was warmed to ambient temperature and stirring was continued for 5 additional $\mathrm{h}$ at which point TLC analysis deemed the reaction was complete. The mixture was diluted with $\mathrm{Et}_{2} \mathrm{O}(20$ $\mathrm{mL})$, washed with water $(1 \times 20 \mathrm{~mL})$, brine $(1 \times 20 \mathrm{~mL})$, dried over $\mathrm{MgSO}_{4}$, filtered and concentrated to give an oil. Flash chromatography of the residue over silica using $20 \rightarrow 25 \%$ EtOAc-hexanes gave $6 \mathbf{j}$ (136 mg, $25 \%$ over 4 steps $)$ as a pure $\left(R_{f}=0.29\right.$, TLC, silica, $20 \%$ EtOAc-hexanes; ${ }^{1} \mathrm{H}$ NMR $(500 \mathrm{MHz}$, $\left.\mathrm{CDCl}_{3}\right)$ ) yellow oil: $[\alpha]^{26}=+8.5\left(c=1.00, \mathrm{CHCl}_{3}\right)$; FT-IR (neat) 2985, 2934, 2861, 1613, 1513, 1463 , 1368, 1247, 1173, 1091, 1034, $820 \mathrm{~cm}^{-1}$; ' $\mathrm{H} \mathrm{NMR}\left(500 \mathrm{MHz}, \mathrm{CDCl}_{3}\right) \delta 7.28$ (app dd, $4 \mathrm{H}, J=7.0,7.0 \mathrm{~Hz}$, $\mathrm{Ar} H$ ), 7.09 (app d, $2 \mathrm{H}, J=8.5 \mathrm{~Hz}, \mathrm{ArH}), 6.87$ (app dd, $2 \mathrm{H}, J=7.0 \mathrm{~Hz}, \mathrm{Ar} H$ ), 6.86 (app dd, $2 \mathrm{H}, J=6.5$ $\mathrm{Hz}, \mathrm{Ar} H$ ), 6.82 (app dd, $2 \mathrm{H}, J=8.5 \mathrm{~Hz}, \mathrm{Ar} H$ ), $4.80\left(\mathrm{~d}, 1 \mathrm{H}, J=10.5 \mathrm{~Hz}, \mathrm{OCH} \mathrm{H}_{2} \mathrm{Ar}\right.$ ), $4.63(\mathrm{~d}, 1 \mathrm{H}, J=11.5$ $\mathrm{Hz}, \mathrm{OCH}_{2} \mathrm{Ar}$ ), 4.57 (d, $\left.1 \mathrm{H}, J=12 \mathrm{~Hz}, \mathrm{OCH} \mathrm{H}_{2} \mathrm{Ar}\right), 4.56\left(\mathrm{~d}, 1 \mathrm{H}, J=11 \mathrm{~Hz}, \mathrm{OCH}_{2} \mathrm{Ar}\right), 4.47$ (d, $1 \mathrm{H}, J=11.5$ $\mathrm{Hz}, \mathrm{OCH}_{2} \mathrm{Ar}$ ), 4.44 (d, $1 \mathrm{H}, J=10 \mathrm{~Hz}, \mathrm{OCH} \mathrm{Ar}_{2}$ ), 4.11 (dddd, $1 \mathrm{H}, J=12.5,12.5,6.5,6.5 \mathrm{~Hz}, H-9$ ), 4.03 (dd, $1 \mathrm{H}, J=7.5,6.0 \mathrm{~Hz}, H-10), 3.79\left(\mathrm{~s}, 3 \mathrm{H}, \mathrm{OCH}_{3}\right), 3.78\left(\mathrm{~s}, 3 \mathrm{H}, \mathrm{OCH}_{3}\right), 3.77\left(\mathrm{~s}, 3 \mathrm{H}, \mathrm{OCH}_{3}\right), 3.69-3.57$ (m, $3 \mathrm{H}, H-1,2 \times H-6), 3.53$ (dd, $1 \mathrm{H}, J=7.5,7.5 \mathrm{~Hz}, H-10), 3.42$ (dd, $1 \mathrm{H}, J=9.5,9.5 \mathrm{~Hz}, H-4), 3.38$ $3.31(\mathrm{~m}, 2 \mathrm{H}, H-3, H-5), 2.11$ (ddd, $1 \mathrm{H}, J=12.5,5.0,1.0 \mathrm{~Hz}, H-2), 1.82-1.69(\mathrm{~m}, 2 \mathrm{H}, H-7, H-8), 1.63-$ $1.56(\mathrm{~m}, 1 \mathrm{H}, H-8), 1.55-1.47(\mathrm{~m}, 1 \mathrm{H}, H-7), 1.43-1.37(\mathrm{~m}, 1 \mathrm{H}, H-2), 1.41\left(\mathrm{~s}, 3 \mathrm{H}, \mathrm{CH}_{3}\right), 1.35$ (s, $3 \mathrm{H}$, $\left.\mathrm{CH}_{3}\right) ;{ }^{13} \mathrm{C}$ NMR $\left(125 \mathrm{MHz}, \mathrm{CDCl}_{3}\right) \delta 159.0,130.6,130.6,130.2,129.5,129.4,129.1,113.6,113.6,113.5$, $80.6,78.8,78.1,75.5,74.9,74.4,72.9,70.9,69.1,68.9,55.1,55.0,36.7,31.2,29.5,26.8,25.6$; HRMS (FAB) calcd for $\mathrm{C}_{37} \mathrm{H}_{48} \mathrm{O}_{9} \mathrm{Na}(\mathrm{M})^{+} 659.3191$, found 659.3192 .

Diol (15j)

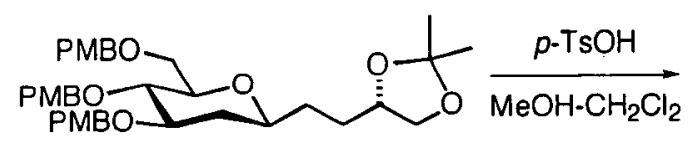

6j

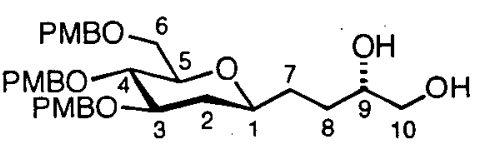

15j 
(C) 2004 American Chemical Society, J. Org. Chem., Postema jo040254t Supporting Info Page 18

Postema, Piper, Betts, Valeriote and Pietraszkewicz RCM-Based Synthesis of a Variety of $\beta$-C-Glycosides and their In Vitro Anti-Solid Tumor Activity

The usual acetonide deprotection was employed using: $\mathrm{CH}_{2} \mathrm{Cl}_{2}(2 \mathrm{~mL}), \mathrm{MeOH}(2 \mathrm{~mL}), p-\mathrm{TsOH}(9 \mathrm{mg}$, $0.047 \mathrm{mmol})$, and sugar acetonide $(100 \mathrm{mg}, 0.157 \mathrm{mmol})$. Typical work-up and flash chromatography of the residue over silica using $2 \rightarrow 4 \% \mathrm{CH}_{2} \mathrm{Cl}_{2}-\mathrm{MeOH}$ gave $15 \mathrm{j}(82 \mathrm{mg}, 88 \%)$ as a pure $\left(\mathrm{R}_{\mathrm{f}}=0.25, \mathrm{TLC}\right.$, silica, $2 \% \mathrm{CH}_{2} \mathrm{Cl}_{2}-\mathrm{MeOH} ;{ }^{1} \mathrm{H}$ NMR $\left.\left(500 \mathrm{MHz}, \mathrm{CDCl}_{3}\right)\right)$ white solid: m.p. $=43-44{ }^{\circ} \mathrm{C} ;[\alpha]^{26}==+6.0(c=$ 1.00, $\mathrm{CHCl}_{3}$ ); FT-IR (neat) 3585 (br), 2934, 2864, 1613, 1513, 1299, 1246, 1174, 1086, 1035, 820, 788, $761 \mathrm{~cm}^{-1}$; ' $\mathrm{H}$ NMR $\left(500 \mathrm{MHz}, \mathrm{CDCl}_{3}\right) \delta$ 7.29-7.24 (m, $\left.4 \mathrm{H}, \mathrm{ArH}\right), 7.10-7.07(\mathrm{~m}, 2 \mathrm{H}, \mathrm{ArH}), 6.88-6.84(\mathrm{~m}$, $4 \mathrm{H}, \mathrm{Ar} H), 6.84-6.80(\mathrm{~m}, 2 \mathrm{H}, \mathrm{Ar} H), 4.78\left(\mathrm{~d}, 1 \mathrm{H}, J=10.5 \mathrm{~Hz}, \mathrm{OCH} \mathrm{Ar}_{2}\right), 4.61(\mathrm{~d}, 1 \mathrm{H}, J=11.5 \mathrm{~Hz}$, $\mathrm{OCH}_{2} \mathrm{Ar}$ ), 4.55 (d, $1 \mathrm{H}, J=11 \mathrm{~Hz}, \mathrm{OCH}_{2} \mathrm{Ar}$ ), 4.50 (d, $1 \mathrm{H}, J=11.5 \mathrm{~Hz}, \mathrm{OCH} \mathrm{Ar}_{2}, 4.44$ (d, $1 \mathrm{H}, J=11.5$ $\mathrm{Hz}, \mathrm{OCH}_{2} \mathrm{Ar}$ ), 4.42 (d, $1 \mathrm{H}, J=10.5 \mathrm{~Hz}, \mathrm{OCH}_{2} \mathrm{Ar}$ ), 3.80 (s, $\left.3 \mathrm{H}, \mathrm{OCH}_{3}\right), 3.80$ (s, $\left.3 \mathrm{H}, \mathrm{OCH}_{3}\right), 3.79$ (s, $3 \mathrm{H}$, $\left.\mathrm{OCH}_{3}\right), 3.72-3.63$ (m, $\left.2 \mathrm{H}, H-3, H-9\right)$, 3.64-3.54 (m, $\left.2 \mathrm{H}, H-1, H-5\right), 3.51$ (dd, $\left.1 \mathrm{H}, J=10,6.0 \mathrm{~Hz}, H-10\right)$, 3.43-3.35 (m, $3 \mathrm{H}, 2 \times H-6, H-10), 3.31(\mathrm{dd}, 1 \mathrm{H}, J=9.5,9.5 \mathrm{~Hz}, H-4), 2.24-2.04(\mathrm{~m}, 2 \mathrm{H}, H-2, \mathrm{O} H)$, 1.80-1.58 (m, $4 \mathrm{H}, H-7,2 \times H-8, \mathrm{OH}), 1.54-1.44(\mathrm{~m}, 1 \mathrm{H}, H-7), 1.45-1.38(\mathrm{~m}, 1 \mathrm{H}, H-2) ;{ }^{13} \mathrm{C}$ NMR (125 $\left.\mathrm{MHz}_{\mathrm{CDCl}}\right) \delta 159.2,159.1,130.6,130.4,130.0,129.7,129.7,129.2,113.8,113.7,80.5,78.5,78.2$, 76.1, 74.6, 73.0, 72.1, 71.2, 69.2, 66.8, 55.3, 37.2, 32.6, 30.8; HRMS (FAB) calcd for $\mathrm{C}_{34} \mathrm{H}_{44} \mathrm{O}_{9} \mathrm{Na}(\mathrm{M})^{+}$ 619.2878 , found 619.2923 .

\section{ENTRY 11}

\section{Ester (3k)}

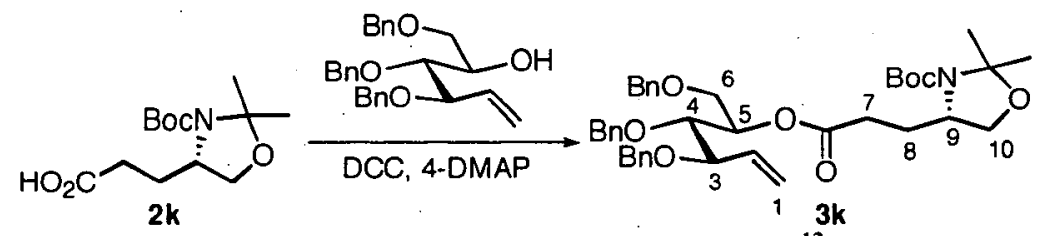

The general method for esterification was followed using: alcohol $1 \mathbf{a}^{13}(256 \mathrm{mg}, 0.61 \mathrm{mmol})$, acid $2 \mathrm{k}$ (200 mg, $0.74 \mathrm{mmol}$ ), DCC (190 mg, $0.92 \mathrm{mmol}$ ), and 4-DMAP (37 mg, $0.3 \mathrm{mmol}$ ) in $\mathrm{CH}_{2} \mathrm{Cl}_{2}$ (4 mL). Flash chromatography of the residue over silica gel using $10 \rightarrow 20 \%$ EtOAc-hexanes gave $3 \mathbf{k}$ ( $402 \mathrm{mg}$, $85 \%)$ as a pure $\left(\mathrm{R}_{\mathrm{f}}=0.49\right.$, TLC, silica, $20 \%$ EtOAc-hexanes; ${ }^{1} \mathrm{H} \mathrm{NMR}\left(500 \mathrm{MHz}, \mathrm{CDCl}_{3}\right)$ ) yellow oil: $[\alpha]_{D}^{26}=+29.1\left(c=1.00, \mathrm{CHCl}_{3}\right) ; \mathrm{FT}-\mathrm{IR}$ (neat) 2976, 2918, 2870, 1735, 1692, 1454, 1387, 1364, 1247 , $1167,1097,1070,736,697 \mathrm{~cm}^{-1} ;{ }^{1} \mathrm{H}$ NMR $\left(500 \mathrm{MHz}, \mathrm{CDCl}_{3}, 45^{\circ} \mathrm{C}\right) \delta 7.33-7.25(\mathrm{~m}, 15 \mathrm{H}, \mathrm{Ph}), 5.90$ (ddd, $1 \mathrm{H}, J=17.5,10.5,8.0 \mathrm{~Hz}, H-2$ ), 5.35-5.27 (m, $2 \mathrm{H}, 2 \times H-1$ ), 5.25 (ddd, $1 \mathrm{H}, J=5.5,5.5,3.5 \mathrm{~Hz}$, $H-5), 4.73\left(\mathrm{~d}, 1 \mathrm{H}, J=11 \mathrm{~Hz}, \mathrm{OCH}_{2} \mathrm{Ph}\right), 4.66\left(\mathrm{~d}, 1 \mathrm{H}, J=11.5 \mathrm{~Hz}, \mathrm{OCH}_{2} \mathrm{Ph}\right), 4.50(\mathrm{~d}, 1 \mathrm{H}, J=12 \mathrm{~Hz}$, $\left.\mathrm{OCH}_{2} \mathrm{Ph}\right), 4.46\left(\mathrm{~d}, 1 \mathrm{H}, J=12.5 \mathrm{~Hz}, \mathrm{OCH}_{2} \mathrm{Ph}\right), 4.35\left(\mathrm{~d}, 1 \mathrm{H}, J=11.5 \mathrm{~Hz}, \mathrm{OCH}_{2} \mathrm{Ph}\right), 3.94(\mathrm{dd}, 1 \mathrm{H}, J=$ 5.0, 5.0 Hz, $H-3$ ), $3.88(\mathrm{dd}, 1 \mathrm{H}, J=8.5,5.5 \mathrm{~Hz}, H-10), 3.86-3.80(\mathrm{~m}, 1 \mathrm{H}, H-9), 3.80-3.77(\mathrm{~m}, 2 \mathrm{H}, 2 \mathrm{x}$ $H-6), 3.72-3.65$ (m, $1 \mathrm{H}, H-10), 3.59$ (d, $1 \mathrm{H}, J=6.0 \mathrm{~Hz}, H-4), 2.30-2.14(\mathrm{~m}, 2 \mathrm{H}, 2$ x H-7), 2.06-1.92 (m, $1 \mathrm{H}, H-8), 1.88-1.78(\mathrm{~m}, 1 \mathrm{H}, H-8), 1.58\left(\mathrm{~s}, 3 \mathrm{H}, \mathrm{CH}_{3}\right), 1.47\left(\mathrm{~s}, 12 \mathrm{H}, 1 \times \mathrm{CH}_{3}, 1 \times\left(\mathrm{CH}_{3}\right)_{3}\right) ;{ }^{13} \mathrm{C} \mathrm{NMR}(125$ $\left.\mathrm{MHz}, \mathrm{CDCl}_{3}\right) \delta 172.0,152.3,138.0,135.3,128.3,128.3,128.2,128.1,127.8,127.7,127.6,119.3,80.5$, 75.0, 73.0, 70.3, 66.7, 56.6, 30.9, 28.7, 28.4, 28.2, 27.6, 26.7, 24.4, 23.1; HRMS (FAB) calcd for $\mathrm{C}_{40} \mathrm{H}_{51} \mathrm{O}_{8} \mathrm{Na}(\mathrm{M})^{+} 696.3507$, found 696.3498 .

\section{C-Glycoside (6k)}

\footnotetext{
${ }^{13}$ Freeman, F.; Robarge, K. D. Carbohydr. Res. 1987, I7I, 1-11.
} 
(C) 2004 American Chemical Society, J. Org. Chem., Postema jo040254t Supporting Info Page 19 RCM-Based Synthesis of a Variety of $\beta-C$-Glycosides and their In Vitro Anti-Solid Tumor Activity
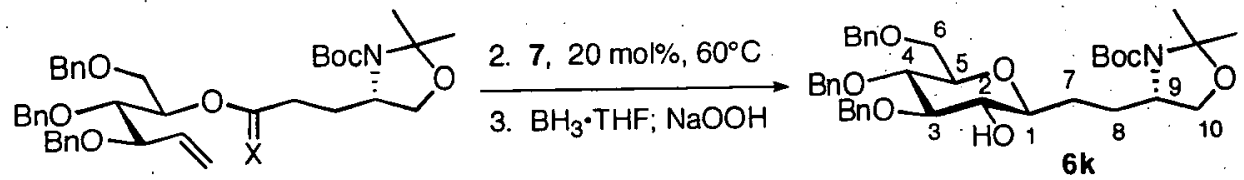

$\mathrm{TiCl}_{4}, \mathrm{Zn}$ dust,
$\mathrm{TMEDA}_{\mathrm{CH}_{2}}, \quad \begin{aligned} & \text { 3k: } \mathrm{X}=\mathrm{O} \\ & \mathrm{PbCl}_{2} \text { (cat.) }\end{aligned} \longrightarrow$ 4k: $\mathrm{X}=\mathrm{CH}_{2}$

The general procedure for the three step protocol was followed using: Titanium tetrachloride (1.27 $\mathrm{mL}, 2$ $\mathrm{M}$ in $\left.\mathrm{CH}_{2} \mathrm{Cl}_{2}, 2.53 \mathrm{mmol}\right)$, THF (2 mL), TMEDA (0.74 mL, $\left.4.89 \mathrm{mmol}\right)$, zinc dust (360 mg, $\left.5.51 \mathrm{mmol}\right)$, lead (II) chloride $(4 \mathrm{mg}, 0.015 \mathrm{mmol})$, ester $3 \mathrm{k}(100 \mathrm{mg}, 0.149 \mathrm{mmol})$, dibromomethane $(97 \mu \mathrm{L}, 1.39$ $\mathrm{mmol}$ ), catalyst $7(31 \mathrm{mg}, 0.037 \mathrm{mmol}, 25 \mathrm{~mol} \%)$, toluene $(12 \mathrm{~mL}), \mathrm{BH}_{3} \cdot \mathrm{THF}(1.5 \mathrm{~mL}, 1 \mathrm{M}$ in THF, 1.5 $\mathrm{mmol}) ; \mathrm{NaOH}(10 \mathrm{~mL}, 1 \mathrm{M}, 10 \mathrm{mmol})$ and hydrogen peroxide $(10 \mathrm{~mL}, 30 \%$ in water, $0.05 \mathrm{~mol})$. After the usual work-up, flash chromatography of the residue over silica using $10 \rightarrow 20 \%$ EtOAc-hexanes gave 6k (49 mg, $50 \%$ over 3 steps) as a pure $\left(R_{f}=0.28\right.$, TLC, silica, 30\% EtOAc-hexanes; ${ }^{1} \mathrm{H} \mathrm{NMR}(500 \mathrm{MHz}$, $\left.\mathrm{CDCl}_{3}\right)$ ) white solid: m.p. $=39-40^{\circ} \mathrm{C} ;[\alpha]_{\mathrm{b}}^{26}==+36.7\left(c=1.00, \mathrm{CHCl}_{3}\right) ;$ FT-IR (neat) $3456,2924,2865$, 2359, 1694, 1454, 1390, 1364, 1255, 1219, 1173, 1093, 1028, 735, $697 \mathrm{~cm}^{-1} ;{ }^{1} \mathrm{H}$ NMR (500 MHz, CDCl $\left.{ }_{3}\right)$ 8 7.29-7.24 (m, $3 \mathrm{H}, \mathrm{ArH}), 7.22-7.18(\mathrm{~m}, 3 \mathrm{H}, \mathrm{Ar} H), 7.17-7.08(\mathrm{~m}, 7 \mathrm{H}, \mathrm{ArH}), 7.07-7.02(\mathrm{~m}, 2 \mathrm{H}, \mathrm{ArH})$, $4.84\left(\mathrm{~d}, 1 \mathrm{H}, \mathrm{J}=12 \mathrm{~Hz}, \mathrm{OCH}_{2} \mathrm{Ph}\right), 4.80-4.70\left(\mathrm{~m}, 2 \mathrm{H}, 2 \times \mathrm{OCH}_{2} \mathrm{Ph}\right), 4.58$ (d, $\left.1 \mathrm{H}, \mathrm{J}=11 \mathrm{~Hz}, \mathrm{OCH}_{2} \mathrm{Ph}\right)$, $4.45\left(\mathrm{~d}, 1 \mathrm{H}, J=12.5 \mathrm{~Hz}, \mathrm{OCH}_{2} \mathrm{Ph}\right), 4.38\left(\mathrm{~d}, 1 \mathrm{H}, J=12.5 \mathrm{~Hz}, \mathrm{OCH}_{2} \mathrm{Ph}\right), 3.98-3.75(\mathrm{~m}, 1 \mathrm{H}, H-9), 3.69-$ $3.63(\mathrm{~m}, 3 \mathrm{H}, 2 \times H-6, H-10), 3.61$ (dd, $1 \mathrm{H}, J=9.5,9.5 \mathrm{~Hz}, H-4), 3.59-3.54(\mathrm{~m}, 1 \mathrm{H}, H-10), 3.43$ (dd $1 \mathrm{H}$, $J=8.5,8.5 \mathrm{~Hz}, H-4) ; 3.34$ (dd, $1 \mathrm{H}, J=9.5,3.5,3.5 \mathrm{~Hz}, H-5), 3.30$ (dd, $1 \mathrm{H}, J=9.0,9.0 \mathrm{~Hz}, H-2)$, 3.16$3.08(\mathrm{~m}, 1 \mathrm{H}, H-1), 2.16-2.06(\mathrm{~s}, 1 \mathrm{H}, \mathrm{OH}), 1.90-1.82(\mathrm{~m}, 1 \mathrm{H}, \mathrm{H}-8), 1.73-1.62\left(\mathrm{~m}, 4 \mathrm{H}, \mathrm{H}-7, \mathrm{CH}_{3}\right), 1.54-$ $1.44\left(\mathrm{~m}, 4 \mathrm{H}, \mathrm{H}-7, \mathrm{CH}_{3}\right), 1.40\left(\mathrm{~s}, 9 \mathrm{H}, \mathrm{CH}_{3}\right) ;{ }^{13} \mathrm{C} \mathrm{NMR}\left(125 \mathrm{MHz}, \mathrm{CDCl}_{3}\right) \delta 139.7,139.4,128.6,128.5$, $128.5,127.9,127.7,127.7,127.6,101.4,87.7,79.9,79.2,75.1,74.8,73.8,70.1,67.5,57.9,31.1,30.1$, $30.1,28.9,28.6,27.6,24.4$; HRMS (FAB) calcd for $\mathrm{C}_{39} \mathrm{H}_{51} \mathrm{O}_{8} \mathrm{Na}(\mathrm{M})^{+} 684.3507$, found 684.3492.

\section{ENTRY 1}

\section{Glycoglycerolipid Synthesis}

\section{C-Glycoglycerolipid (17a)}

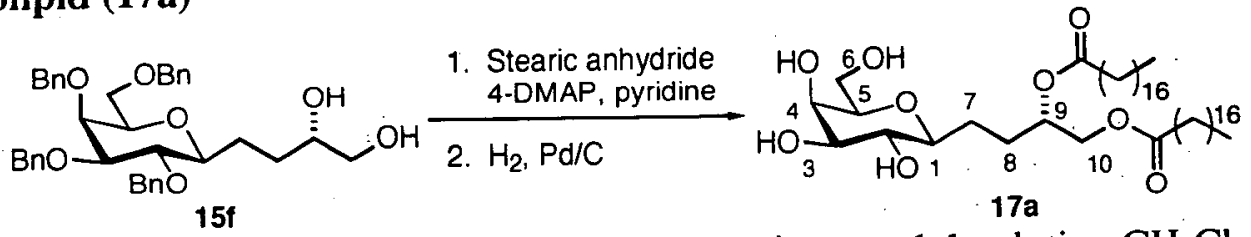

Stearic anhydride $(172 \mathrm{mg}, 0.31 \mathrm{mmol})$ was added in one portion to a $1: 1$ solution $\mathrm{CH}_{2} \mathrm{Cl}_{2}$-pyridine $(1: 1,2$ $\mathrm{mL}$ ) containing diol $15 \mathrm{f}(32 \mathrm{mg}, 0.052 \mathrm{mmol})$ and 4-DMAP ( $3 \mathrm{mg}, 0.03 \mathrm{mmol})$ and the solution was stirred for $18 \mathrm{hrs}$ at ambient temperature. The resulting orange solution was then concentrated and flash chromatography of the residue over silica using $10 \rightarrow 20 \%$ EtOAc-hexanes gave the product $16 \mathbf{a}$ ( $46 \mathrm{mg}$, $77 \%)$ as a pure $\left(\mathrm{R}_{\mathrm{f}}=0.34\right.$, TLC, silica, $20 \%$ EtOAc-hexanes; ${ }^{1} \mathrm{H}$ NMR (500 $\left.\mathrm{MHz} \mathrm{CDCl}_{3}\right)$; m.p. $=34-35$ ${ }^{\circ} \mathrm{C}$; HRMS (FAB) calcd for $\mathrm{C}_{74} \mathrm{H}_{112} \mathrm{O}_{9} \mathrm{Na}(\mathrm{M})^{+} 1167.8199$, found 1167.8196$)$ white solid that was directly carried onto the next step. $\mathrm{Pd} / \mathrm{C}(5 \mathrm{mg})$ was added in one portion to a solution of $16 \mathrm{a}(15 \mathrm{mg}, 13.0 \mu \mathrm{mol})$ in EtOAc-MeOH $(1: 1,5 \mathrm{~mL})$. The solution was degassed five times with $\mathrm{H}_{2}$ and the resulting suspension shaken under an atmosphere of $\mathrm{H}_{2}(60 \mathrm{psi})$ for 15 hours at ambient temperature. The reaction mixture was filtered through Celite using a mixture of $\mathrm{MeOH}-\mathrm{CH}_{2} \mathrm{Cl}_{2}(1: 1,40 \mathrm{~mL})$ as the eluent and the resulting solution concentrated. Flash chromatography of the residue over silica using $5 \rightarrow 10 \% \mathrm{MeOH}-\mathrm{CH}_{2} \mathrm{Cl}_{2}$ gave $17 \mathrm{a}(21 \mathrm{mg}, 78 \%)$ as a pure $\left(\mathrm{R}_{\mathrm{f}}=0.24\right.$, TLC, silica, $10 \% \mathrm{MeOH}-\mathrm{CH}_{2} \mathrm{Cl}_{2} ;{ }^{1} \mathrm{H} \mathrm{NMR}(500 \mathrm{MHz}$, $\left.\mathrm{CDCl}_{3}\right)$ ) white solid: m.p. $=71-72{ }^{\circ} \mathrm{C} ;[\alpha]_{\mathrm{b}}^{26}=-7.7\left(c=1.00, \mathrm{CHCl}_{3}\right)$; FT-IR (neat) $3370(\mathrm{br}), 2958,2859$, 
(C) 2004 American Chemical Society, J. Org. Chem., Postema jo040254t Supporting Info Page 20

$1729,1704,1468,1379,788,724 \mathrm{~cm}^{-1} ;{ }^{1} \mathrm{H} \mathrm{NMR}\left(500 \mathrm{MHz}, \mathrm{CDCl}_{3}\right) \delta 5.07$ (dddd, $1 \mathrm{H}, J=7.5,7.5,7.5$, $4.5 \mathrm{~Hz}, H-9$ ), 4.15 (dd, $1 \mathrm{H}, J=12,3.0 \mathrm{~Hz}, H-10$ ), 3.92 (dd, $1 \mathrm{H}, J=12,7.0 \mathrm{~Hz}, H-10), 3.85-3.82$ (m, 1 $\mathrm{H}, H-4), 3.70$ (dd, $1 \mathrm{H}, J=11.5,6.0 \mathrm{~Hz}, H-6), 3.61(\mathrm{dd}, 1 \mathrm{H}, J=11.5,5.0, H-6), 3.35-3.26(\mathrm{~m}, 3 \mathrm{H}, H-2$, $H-3, H-5), 3.02$ (ddd, $1 \mathrm{H}, J=8.5,8.5,2.0 \mathrm{~Hz}, H-1), 2.22-2.16\left(\mathrm{~m}, 4 \mathrm{H}, 2 \mathrm{x}-\mathrm{C}(=\mathrm{O}) \mathrm{CH}_{2^{-}}\right), 1.84-1.75(\mathrm{~m}, 1$ $\mathrm{H}, H-7), 1.75-1.68(\mathrm{~m}, 1 \mathrm{H}, \mathrm{H}-8), 1.63-1.55(\mathrm{~m}, 1 \mathrm{H}, \mathrm{H}-8), 1.54-1.45(\mathrm{~m}, 4 \mathrm{H}, 2$ x CH$), 1.45-1.38(\mathrm{~m}, 1$ $\mathrm{H}, \mathrm{H}-7$ ), 1.21-1.13 (bs, $56 \mathrm{H}, 28 \times \mathrm{CH}_{2}$ ), 0.77 (app t, $\left.6 \mathrm{H}, J=7.0 \mathrm{~Hz}, 2 \times \mathrm{CH}_{3}\right) ;{ }^{13} \mathrm{C}$ NMR (125 MHz, $\left.\mathrm{CDCl}_{3}\right) \delta 173.9,79.2,77.8,74.9,71.2,71.1,69.5,64.9,62.0,34.3,34.0,31.7,29.5,29.5,29.3,29.2,29.1$, 29.0, 26.7, 26.6, 24.8, 24.7, 22.5, 13.9; HRMS (FAB) calcd for $\mathrm{C}_{46} \mathrm{H}_{88} \mathrm{O}_{9} \mathrm{Na}(\mathrm{M})^{+} 807.6321$, found 807.6357 .

\section{ENTRY 2}

\section{C-Glycoglycerolipid (17b) ${ }^{14}$}

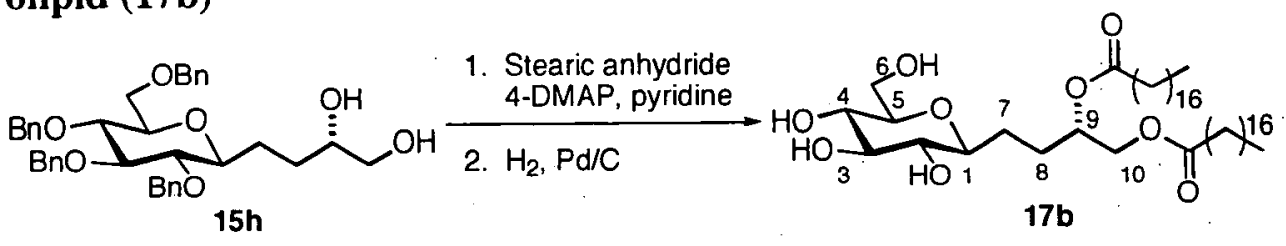

\section{ENTRY 3}

\section{C-Glycoglycerolipid (17c)}
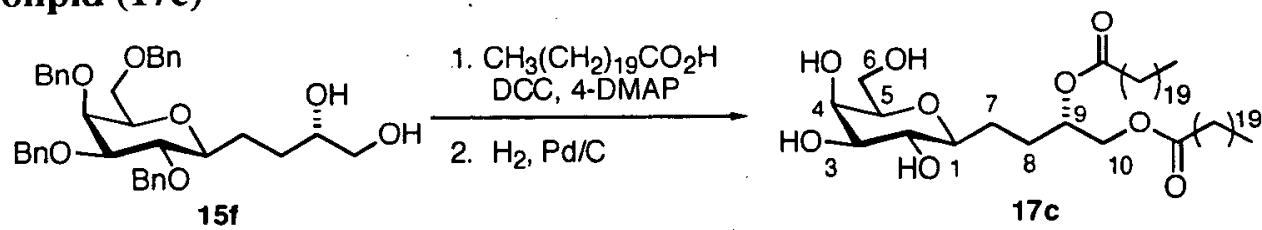

The general method for DCC-mediated esterification was followed using: Diol $15 f(20 \mathrm{mg}, 0.033 \mathrm{mmol})$, heneicosanoic acid ( $37 \mathrm{mg}, 0.11 \mathrm{mmol}$ ), DCC (10.2 $\mathrm{mg}, 0.05 \mathrm{mmol}$ ), 4-DMAP (2 mg, $0.017 \mathrm{mmol})$ and $\mathrm{CH}_{2} \mathrm{Cl}_{2}(2 \mathrm{~mL})$. After the usual work-up, flash chromatography of the residue over silica using $10 \rightarrow 20 \%$ EtOAc-hexanes gave $16 \mathrm{c}(31 \mathrm{mg}, 76 \%)$ as a pure $\left(\mathrm{R}_{\mathrm{f}}=0.32\right.$, TLC, silica, $15 \%$ EtOAc-hexanes; ${ }^{1} \mathrm{H}$ NMR $\left(500 \mathrm{MHz}, \mathrm{CDCl}_{3}\right)$, HRMS (FAB) calcd for $\mathrm{C}_{80} \mathrm{H}_{124} \mathrm{O}_{9} \mathrm{Na}(\mathrm{M})^{+} 1251.9138$, found 1251.9081) white solid. The product was then taken directly to the next step and the general method of hydrogenolysis was followed using 16c (25 mg, $0.02 \mathrm{mmol}), \mathrm{Pd} / \mathrm{C}(10 \mathrm{mg})$ and EtOAc $(4 \mathrm{~mL})$. The usual work-up followed by flash chromatography of the residue over silica using $5 \rightarrow 10 \% \mathrm{MeOH}-\mathrm{CH}_{2} \mathrm{Cl}_{2}$ gave 17c (13.4 mg, $79 \%)$ as a pure $\left(\mathrm{R}_{\mathrm{f}}=0.22\right.$, TLC, silica, $10 \% \mathrm{MeOH}-\mathrm{CH}_{2} \mathrm{Cl}_{2} ;{ }^{1} \mathrm{H} \mathrm{NMR}\left(500 \mathrm{MHz}, \mathrm{CDCl}_{3}\right)$ ) white solid: m.p. $=75-76^{\circ} \mathrm{C} ;\left[\alpha b_{b}^{26}=-5.5\left(c=1.00, \mathrm{CHCl}_{3}\right)\right.$; FT-IR (neat) 3404 (br), 2917, 2950, 1737, 1467, 1243, $1168,1103,721 \mathrm{~cm}^{-1} ;{ }^{1} \mathrm{H}$ NMR $\left(500 \mathrm{MHz} \mathrm{CDCl}_{3}\right) \delta 5.16-5.10(\mathrm{~m}, 1 \mathrm{H}, H-9), 4.20$ (dd, $1 \mathrm{H}, J=11.5,3.0$ $\mathrm{Hz}, H-10), 3.97$ (dd, $1 \mathrm{H}, J=12.0,7.0 \mathrm{~Hz}, H-10$ ), 3.90 (app s, $1 \mathrm{H}, H-4$ ), 3.76 (dd, $1 \mathrm{H}, J=11.5,6.0 \mathrm{~Hz}$, $H-6), 3.67$ (dd, $1 \mathrm{H}, J=11.5,5.0 \mathrm{~Hz}, H-6), 3.42-3.32$ (m, $3 \mathrm{H}, H-2, H-3, H-5), 3.09$ (ddd, $1 \mathrm{H}, J=9.0$, 9.0, 2.0 Hz, H-1), 2.27-2.21 (m, $\left.4 \mathrm{H}, 2 \times-\mathrm{C}(=\mathrm{O}) \mathrm{CH}_{2^{-}}\right), 1.89-1.81(\mathrm{~m}, 1 \mathrm{H}, H-7), 1.80-1.72(\mathrm{~m}, 1 \mathrm{H}, H-8)$, $1.68-1.58(\mathrm{~m}, 1 \mathrm{H}, H-8), 1.58-1.50\left(\mathrm{~m}, 4 \mathrm{H}, 2 \times \mathrm{CH}_{2}\right), 1.50-1.44(\mathrm{~m}, 1 \mathrm{H}, H-7), 1.26-1.19(\mathrm{~m}, 68 \mathrm{H}, 34 \mathrm{x}$ $\left.\mathrm{CH}_{2}\right), 0.82\left(\mathrm{t}, 6 \mathrm{H}, J=7.0 \mathrm{~Hz}, 2 \times \mathrm{CH}_{3}\right) ;{ }^{13} \mathrm{C} \mathrm{NMR}\left(125 \mathrm{MHz}, \mathrm{CDCl}_{3}\right) \delta 174.0,173.9,79.2,77.8,75.0$, $71.3,71.2,69.7,64.9,62.3,34.4,34.1,31.8,29.6,29.5,29.4,29.4,29.2,29.2,29.2,29.0,26.7,24.9,24.7$, 22.6, 14.0; HRMS (FAB) calcd for $\mathrm{C}_{52} \mathrm{H}_{100} \mathrm{O}_{9} \mathrm{Na}(\mathrm{M})^{+} 891.7260$, found 891.7267.

\footnotetext{
14 For full experimental and spectral data listing for this compound please see the supporting information corresponding to: Postema, M. H. D.; Piper, J. L. Org. Lett. 2003, 5, 1721-1723.
} 
(C) 2004 American Chemical Society, J. Org. Chem., Postema jo040254t Supporting Info Page 21 RCM-Based Synthesis of a Variety of $\beta-C$-Glycosides and their In Vitro Anti-Solid Tumor Activity

\section{ENTRY 4}

\section{C-Glycoglycerolipid (17d)}

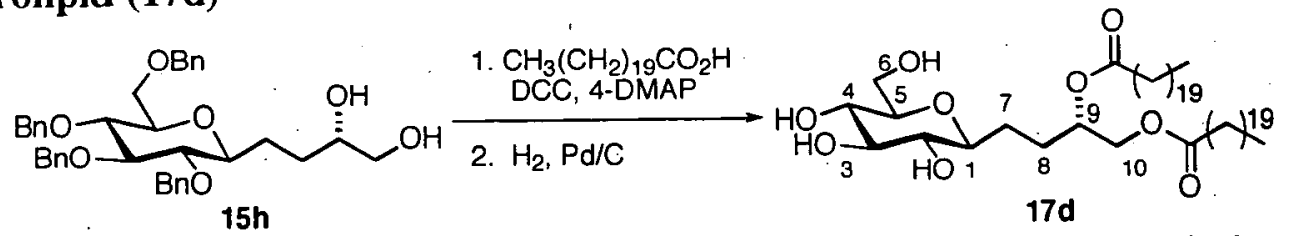

The general procedure for esterification was employed using $15 \mathrm{~h}$ (20 $\mathrm{mg}, 0.033 \mathrm{mmol})$, heneicosanoic acid (37 mg, $0.11 \mathrm{mmol}$ ), 4-DMAP ( $5 \mathrm{mg}, 0.41 \mathrm{mmol}$ ), and DCC (10 mg, $49.5 \mu \mathrm{mol})$. Flash chromatography of the residue on silica gel using $20 \%$ EtOAc-hexanes gave $16 \mathrm{~d}(31.0 \mathrm{mg}, 77 \%)$ as a pure $\left(\mathrm{R}_{\mathrm{f}}=0.41\right.$, TLC, silica, $20 \%$ EtOAc-hexanes; ${ }^{1} \mathrm{H}$ NMR $\left(500 \mathrm{MHz}, \mathrm{CDCl}_{3}\right)$; HRMS (FAB) calcd for $\mathrm{C}_{80} \mathrm{H}_{124} \mathrm{O}_{9} \mathrm{Na}(\mathrm{M})^{+} 1251.9138$, found 1251.9124) oil. The product was then taken onto the next step and the general procedure for hydrogenolysis was followed using: $16 \mathrm{~d}(25 \mathrm{mg}, 20 \mu \mathrm{mol})$, EtOAc-MeOH $(1: 1$, $10 \mathrm{~mL})$ and $\mathrm{Pd} / \mathrm{C}(5 \mathrm{mg})$. After the usual work-up, flash chromatography of the residue over silica using $10 \% \mathrm{MeOH}-\mathrm{CH}_{2} \mathrm{Cl}_{2}$ gave $17 \mathrm{~d}(15.2 \mathrm{mg}, 86 \%)$ as a pure $\left(\mathrm{R}_{\mathrm{f}}=0.25, \mathrm{TLC}\right.$, silica, $10 \% \mathrm{MeOH}-\mathrm{CH}_{2} \mathrm{Cl}_{2} ;{ }^{1} \mathrm{H}$ NMR $\left.\left(500 \mathrm{MHz}, \mathrm{CDCl}_{3}\right)\right]$ white solid: m.p. $=40-41^{\circ} \mathrm{C} ;\left[\alpha b^{26}=+30.1\left(c=1.00, \mathrm{CH}_{2} \mathrm{Cl}_{2}\right)\right.$; FT-IR (neat) $3451,2920,2851,1714,1612,1513,1360,1092,1036,821 \mathrm{~cm}^{-1} ;{ }^{1} \mathrm{H} \mathrm{NMR}\left(500 \mathrm{MHz}, \mathrm{CDCl}_{3}\right) \delta 5.05$ (dddd, $1 \mathrm{H}, J=8.0,8.0,8.0,4.5 \mathrm{~Hz}, H-9$ ), 4.15 (dd, $1 \mathrm{H}, J=12.5,3.0 \mathrm{~Hz}, H-10$ ), 3.91 (dd, $1 \mathrm{H}, J=11.5$, $7.0 \mathrm{~Hz}, H-10), 3.69$ (dd, $1 \mathrm{H}, J=12.5,3.5 \mathrm{~Hz}, H-6), 3.61$ (dd, $1 \mathrm{H}, J=12,5.0 \mathrm{~Hz}, H-6), 3.28-3.22(\mathrm{~m}, 2$ $\mathrm{H}, H-3, H-4), 3.12-3.08(\mathrm{~m}, 1 \mathrm{H}, H-5), 3.06$ (ddd, $1 \mathrm{H}, J=8.5,8.5,2.0 \mathrm{~Hz}, H-1), 3.02-2.96(\mathrm{~m}, 1 \mathrm{H}, H-2)$, 2.23-2.15 (m, $\left.4 \mathrm{H}, 2 \times \mathrm{C}(=\mathrm{O}) \mathrm{CH}_{2}-\right), 1.82-1.74(\mathrm{~m}, 1 \mathrm{H}, \mathrm{H}-7), 1.74-1.66(\mathrm{~m}, 1 \mathrm{H}, H-8), 1.60-1.53(\mathrm{~m}, 1 \mathrm{H}$, H-7), 1.53-1.44 (m, $\left.4 \mathrm{H}, 2 \times \mathrm{CH}_{2}\right), 1.41-1.33(\mathrm{~m}, 1 \mathrm{H}, H-7), 1.20-1.16(\mathrm{~m}, \mathrm{H}, H-7), 1.30-1.22(\mathrm{~m}, 52 \mathrm{H}$, $\left.26 \times \mathrm{CH}_{2}\right), 0.76\left(\mathrm{t}, 6 \mathrm{H}, J=7.0 \mathrm{~Hz}, 2 \times \mathrm{CH}_{3}\right) ;{ }^{13} \mathrm{C} \mathrm{NMR}\left(125 \mathrm{MHz}, \mathrm{CDCl}_{3}\right) \delta 174.0,173.9,79.2,78.8$, 78.5, 73.4, 71.1 , 70.0, 65.1, 61.8, 34.5, 34.2, 31.9, 29.7, 29.7, 29.6, 29.6, 29.4, 29.2 26.6, 26.6, 25.0, 24.8, 22.7, 14.1; HRMS (FAB) calcd for $\mathrm{C}_{52} \mathrm{H}_{100} \mathrm{O}_{9} \mathrm{Na}(\mathrm{M})^{+} 891.7260$, found 891.7260 .

\section{ENTRY 5}

\section{C-Glycoglycerolipid (17e)}
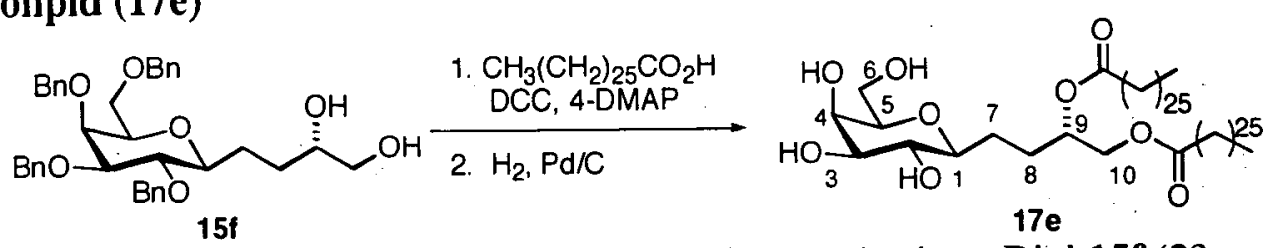

The general method for DCC-mediated esterification was followed using: Diol $\mathbf{1 5 f}(23 \mathrm{mg}, 0.038 \mathrm{mmol})$, 4-DMAP (2.3 mg, $0.019 \mathrm{mmol})$, heptacosanoic acid (53 mg, $0.13 \mathrm{mmol}$ ) and DCC (11.8 mg, 0.057 $\mathrm{mmol})$. Flash chromatography of the residue over silica using $10 \rightarrow 20 \%$ EtOAc-hexanes gave 16e (43 $\mathrm{mg}, 0.03 \mathrm{mmol}, 81 \%)$ as a pure $\left(\mathrm{R}_{\mathrm{f}}=0.35\right.$, TLC, silica, $15 \%$ EtOAc-hexanes; ' $\mathrm{H}$ NMR $(500 \mathrm{MHz}$, $\mathrm{CDCl}_{3}$ ); HRMS (FAB) calcd for $\mathrm{C}_{92} \mathrm{H}_{148} \mathrm{O}_{9} \mathrm{Na}(\mathrm{M})^{+} 1421.1049$, found 1421.1003) white solid. The product was then taken directly to the next step and the general method of hydrogenolysis was followed using 16e (25 mg, $0.02 \mathrm{mmol}), \mathrm{Pd} / \mathrm{C}(10 \mathrm{mg})$ and EtOAc $(4 \mathrm{~mL})$. After the usual work-up, flash chromatography of the residue over silica using $5 \rightarrow 10 \% \mathrm{MeOH}-\mathrm{CH}_{2} \mathrm{Cl}_{2}$ gave $17 \mathrm{e}(14 \mathrm{mg}, 80 \%)$ as a pure $\left(\mathrm{R}_{\mathrm{f}}=0.33\right.$, TLC, silica, $10 \% \mathrm{MeOH}-\mathrm{CH}_{2} \mathrm{Cl}_{2} ;{ }^{1} \mathrm{H}$ NMR $\left.\left(500 \mathrm{MHz}, \mathrm{CDCl}_{3}\right)\right)$ white solid: m.p. $=84-85^{\circ} \mathrm{C}$; $[\alpha]^{26}=+12.1\left(c=1.00, \mathrm{CHCl}_{3}\right)$; FT-IR (neat) 3363, 2916, 2848, 1708, 1464, 1356, 1219, 1091, $720 \mathrm{~cm}^{-1}$; ${ }^{1} \mathrm{H}$ NMR (500 MHz, CDCl $)_{3} \delta 5.21-5.15(\mathrm{~m}, 1 \mathrm{H}, H-9), 4.25(\mathrm{dd}, 1 \mathrm{H}, J=11.5,3.5 \mathrm{~Hz}, H-10), 4.02$ (dd, 1 $\mathrm{H}, J=12.0,7.0 \mathrm{~Hz}, H-10), 3.95$ (dd, $1 \mathrm{H}, J=2.0,2.0 \mathrm{~Hz}, H-4), 3.82$ (dd, $1 \mathrm{H}, J=12.0,6.0 \mathrm{~Hz}, H-6), 3.76$ (ddd, $1 \mathrm{H}, J=10.5,10.5,3.5 \mathrm{~Hz}, H-6), 3.44$ (dd, $1 \mathrm{H}, J=9.0,9.0 \mathrm{~Hz}, H-2), 3.43-3.39$ (m, $2 \mathrm{H}, H-3, H-5$ ), 
(C) 2004 American Chemical Society, J. Org. Chem., Postema jo040254t Supporting Info Page 22

3.14 (ddd, $1 \mathrm{H}, J=8.0,8.0,2.5 \mathrm{~Hz}, H-1) ; 2.31-2.26\left(\mathrm{~m}, 4 \mathrm{H}, 2 \times \mathrm{CH}_{2}\right), 1.95-1.86(\mathrm{~m}, 1 \mathrm{H}, H-7), 1.85-1.75$ (m, $1 \mathrm{H}, \mathrm{H}-8), 1.75-1.67$ (m, $1 \mathrm{H}, \mathrm{H}-8), 1.64-1.53\left(\mathrm{~m}, 5 \mathrm{H}, \mathrm{H}-7,2 \times \mathrm{CH}_{2}\right), 1.34-1.24$ (m, $92 \mathrm{H}, 46 \times \mathrm{CH}_{2}$ ), $0.77\left(\mathrm{t}, 6 \mathrm{H}, J=6.5 \mathrm{~Hz}, 2 \times \mathrm{CH}_{3}\right) ;{ }^{13} \mathrm{C}$ NMR $\left(125 \mathrm{MHz}, \mathrm{CDCl}_{3}\right) \delta 173.8,173.7,79.3,77.9,77.8,75.3$, $71.4,70.3,71.4,70.3,65.0,62.9,34.4,34.1,31.8,29.6,29.4,29.4,29.4,29.3,29.2,29.2,29.2,29.1,26.8$, 24.9, 24.8, 23.9, 22.6, 13.9; HRMS (FAB) calcd for $\mathrm{C}_{64} \mathrm{H}_{124} \mathrm{O}_{9} \mathrm{Na}(\mathrm{M})^{+} 1059.9138$, found 1059.9124 .

\section{ENTRY 6}

\section{C-Glycoglycerolipid (17f)}

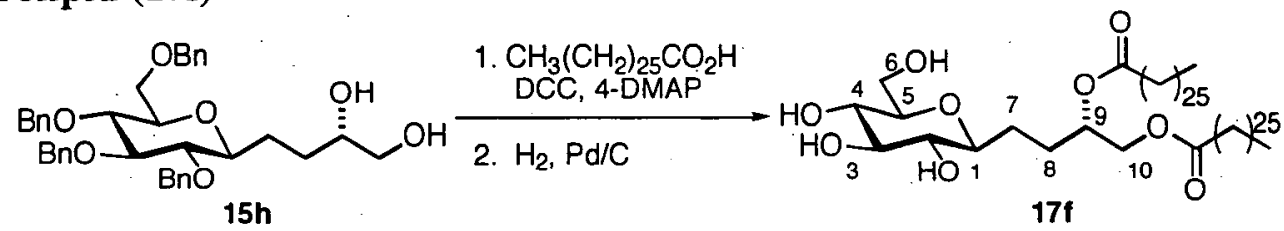

The general procedure for DCC-mediated esterification was followed using: diol $15 \mathrm{~h}$ ( $18 \mathrm{mg}, 0.03$ mmol), heptacosanoic acid ( $18 \mathrm{mg}, 0.044 \mathrm{mmol}), 4$-DMAP $(2 \mathrm{mg}, 0.015 \mathrm{mmol})$ and DCC ( $9 \mathrm{mg}, 0.044$ mmol). Flash chromatography using 20\% EtOAc-hexanes gave $16 \mathbf{f}(33.0 \mathrm{mg}, 81 \%)$ as a pure $\left(\mathrm{R}_{\mathrm{f}}=0.40\right.$, TLC; silica, 20\% EtOAc-hexanes; ${ }^{1} \mathrm{H}$ NMR (500 MHz, CDCl $)$; HRMS (FAB) calcd for $\mathrm{C}_{92} \mathrm{H}_{148} \mathrm{O}_{9} \mathrm{Na}$ $(\mathrm{M})^{+} 1421.1049$, found 1421.1125 ) yellow oil. The product was then taken directly to the next step and the general method of hydrogenolysis was followed using: $16 \mathrm{f}(10 \mathrm{mg}, 6.6 \mu \mathrm{mol})$ in EtOAc:MeOH (1:1, $5 \mathrm{~mL}$ ) was added $\mathrm{Pd} / \mathrm{C}(2 \mathrm{mg})$. After the usual work-up, flash chromatography of the residue over silica gel using $5 \rightarrow 10 \% \mathrm{MeOH}-\mathrm{CH}_{2} \mathrm{Cl}_{2}$ gave $17 \mathrm{f}(5 \mathrm{mg}, 71 \%)$ as a pure $\left(\mathrm{R}_{\mathrm{f}}=0.31, \mathrm{TLC}\right.$, silica, $10 \% \mathrm{MeOH}-$ $\mathrm{CH}_{2} \mathrm{Cl}_{2}$; 'H NMR $\left.\left(500 \mathrm{MHz}, \mathrm{CDCl}_{3}\right)\right]$ white solid: m.p. $=99-100^{\circ} \mathrm{C}$; $[\alpha]_{\mathrm{b}}^{26}=+2.9\left(c=1.00, \mathrm{CHCl}_{3}\right) ; \mathrm{FT}-$ IR (neat) 3486 (br), 2916, 2850, 2361, 1713, 1612, 1513, 1465, 1360, 1247, 1219, 1090, 1035, $820 \mathrm{~cm}^{-1}$; 'H NMR (500 MHz, $\left.\mathrm{CDCl}_{3}\right) \delta 5.18-5.12(\mathrm{~m}, 1 \mathrm{H}, \mathrm{H}-9), 4.23(\mathrm{dd}, 1 \mathrm{H}, J=12.5,3.0 \mathrm{~Hz}, \mathrm{H}-10), 3.98$ (dd, 1 $\mathrm{H}, J=12.0,7.0 \mathrm{~Hz}, H-10), 3.78$ (dd, $1 \mathrm{H}, J=12.5,3.0 \mathrm{~Hz}, H-6), 3.71$ (dd, $1 \mathrm{H}, J=12.0,4.5 \mathrm{~Hz}, H-6$ ), 3.44-3.35 (m, $2 \mathrm{H}, H-3, H-4), 3.24-3.18$ (m, $2 \mathrm{H}, H-1, H-5$ ), 3.13 (dd, $1 \mathrm{H}, J=8.5,8.5 \mathrm{~Hz}, H-2$ ), 2.302.24 (m, $\left.4 \mathrm{H}, \mathrm{C}(\mathrm{O}) \mathrm{CH}_{2}-\right), 2.21-2.13(\mathrm{~m}, 4 \mathrm{H}, 4 \times \mathrm{OH}), 1.90-1.82(\mathrm{~m}, 1 \mathrm{H}, H-7), 1.80-1.71(\mathrm{~m}, 1 \mathrm{H}, \mathrm{H}-8)$, 1.70-1.62 (m, $1 \mathrm{H}, H-8), 1.62-1.52\left(\mathrm{~m}, 4 \mathrm{H}, 2 \times \mathrm{CH}_{2}\right), 1.52-1.43(\mathrm{~m}, 1 \mathrm{H}, H-7), 1.30-1.21(\mathrm{~m}, 92 \mathrm{H}, 46 \mathrm{x}$ $\left.\mathrm{CH}_{2}\right), 0.85\left(\mathrm{t}, 6 \mathrm{H}, J=7.0 \mathrm{~Hz}, 2 \times \mathrm{CH}_{3}\right) ;{ }^{13} \mathrm{C}$ NMR $\left(125 \mathrm{MHz}, \mathrm{CDCl}_{3}\right) \delta 173.9,173.9,79.2,78.7,77.2$, 77.2, 73.4, 64.8, 61.9, 34.3, 34.0, 31.7, 29.5, 29.3, 29.2, 29.1, 28.9, 26.7, 26.4, 24.8, 24.7, 22.5, 13.9; HRMS (FAB) calcd for $\mathrm{C}_{64} \mathrm{H}_{124} \mathrm{O}_{9} \mathrm{Na}(\mathrm{M})^{+} 1059.9138$, found 1059.9102 .

\section{ENTRY 7}

\section{C-Glycoglycerolipid (17g)}

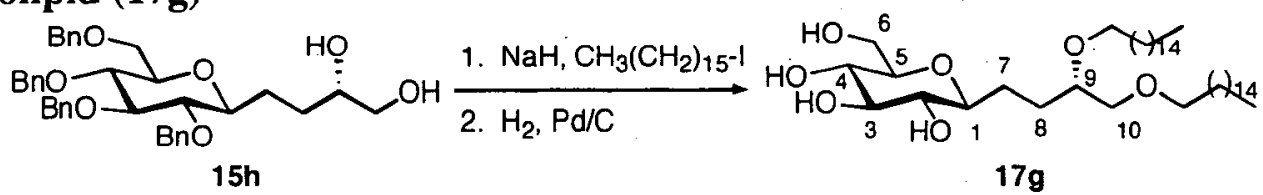

1-Iodohexadecane ( $50 \mu \mathrm{L}, 0.16 \mathrm{mmol})$ was added dropwise over $10 \mathrm{~min}$ to a DMF (1 mL) solution of diol $15 \mathrm{~h}(25 \mathrm{mg}, 0.04 \mathrm{mmol})$ and washed $\mathrm{NaH}(2 \mathrm{mg}, 0.09 \mathrm{mmol})$ at $0{ }^{\circ} \mathrm{C}$. After $10 \mathrm{~min}$, the usual work-up was followed by flash chromatography over silica gel using $20 \% \mathrm{Et}_{2} \mathrm{O}$-hexanes to give $16 \mathrm{~g}(8 \mathrm{mg}, 7.53$ $\mu \mathrm{mol}, 19 \%)$ as a pure $\left(\mathrm{R}_{\mathrm{f}}=0.40\right.$, TLC, silica, $20 \% \mathrm{Et}_{2} \mathrm{O}$-hexanes; ${ }^{1} \mathrm{H}$ NMR $\left(500 \mathrm{MHz}, \mathrm{CDCl}_{3}\right) ; \mathrm{HRMS}$ (FAB) calcd for $\mathrm{C}_{70} \mathrm{H}_{108} \mathrm{O}_{7} \mathrm{Na}(\mathrm{M})^{+} 1083.7987$, found 1083.8018) clear oil. The product was then taken directly to the next step and the general method of hydrogenolysis was followed using: $16 \mathrm{~g}(16 \mathrm{mg}, 15.0$ $\mu \mathrm{mol})$ in EtOAc: $\mathrm{MeOH}(1: 1,5 \mathrm{~mL})$ was added $\mathrm{Pd} / \mathrm{C}(10 \mathrm{mg})$. Flash chromatography of the residue over silica gel using $5 \rightarrow 10 \% \mathrm{MeOH}-\mathrm{CH}_{2} \mathrm{Cl}_{2}$ gave $17 \mathrm{~g}(8 \mathrm{mg}, 76 \%)$ as a pure $\left(\mathrm{R}_{\mathrm{f}}=0.20, \mathrm{TLC}\right.$, silica, $10 \%$ 
(C) 2004 American Chemical Society, J. Org. Chem., Postema jo040254t Supporting Info Page 23

MeOH-CH $\mathrm{Cl}_{2} ;{ }^{1} \mathrm{H}$ NMR $\left.\left(500 \mathrm{MHz}, \mathrm{CDCl}_{3}\right)\right]$ white solid: m.p. $=64-65^{\circ} \mathrm{C} ;[\alpha]^{23}{ }_{\mathrm{D}}=+7.2(c=1.00$, $\mathrm{CH}_{2} \mathrm{Cl}_{2}$ ); FT-IR (neat) $3353,2921,2851,1730,1462,1376,1248,1102,720 \mathrm{~cm}^{-1} ;{ }^{1} \mathrm{H}$ NMR $(500 \mathrm{MHz}$, $\mathrm{CDCl}_{3}$ ) $\delta 3.77$ (dd, $\left.1 \mathrm{H}, J=12.5,3.5 \mathrm{~Hz}, H-6\right), 3.64$ (dd, $\left.1 \mathrm{H}, J=12,5.0 \mathrm{~Hz}, H-6\right), 3.55$ (ddd, $1 \mathrm{H}, J=$ 9.5, $6.5 \mathrm{~Hz}, 6.5 \mathrm{~Hz}, H-9), 3.44-3.30\left(\mathrm{~m}, 9 \mathrm{H}, H-2, H-3, H-4,2 \times H-10,4 \times \mathrm{CH}_{2}\right), 3.22-3.15(\mathrm{~m}, 1 \mathrm{H}, H-5)$, 3.12 (dd, $1 \mathrm{H}, J=10,10 \mathrm{~Hz}, H-1), 2.65-2.62(\mathrm{bs}, 4 \mathrm{H}, 4 \mathrm{x} \mathrm{OH}), 1.85-1.77(\mathrm{~m}, 1 \mathrm{H}, H-7), 1.64-1.47$ (m, 7 $\left.\mathrm{H}, \mathrm{H}-7, \mathrm{H}-8,2 \times \mathrm{CH}_{2}\right), 1.29-1.18\left(\mathrm{bs}, 52 \mathrm{H}, 26 \times \mathrm{CH}_{2}\right), 0.83\left(\mathrm{t}, 6 \mathrm{H}, \mathrm{J}=7.0 \mathrm{~Hz}, 2 \times \mathrm{CH}_{3}\right) ;{ }^{13} \mathrm{C}$ NMR $(125$ $\left.\mathrm{MHz}, \mathrm{CDCl}_{3}\right) \delta 79.0,78.9,78.6,78.3,73.3,73.2,71.7,70.6,70.5,62.3,31.8,30.0,29.6,29.6,29.5,29.4$, 29.4, 29.3, 27.3, 27.2, 26.0, 22.6, 14.0; HRMS (FAB) calcd for $\mathrm{C}_{42} \mathrm{H}_{84} \mathrm{O}_{7} \mathrm{Na}(\mathrm{M}+\mathrm{Na})^{+} .723 .6109$, found 723.6068 .

\section{ENTRY 8}

\section{C-Glycoglycerolipid (17h)}

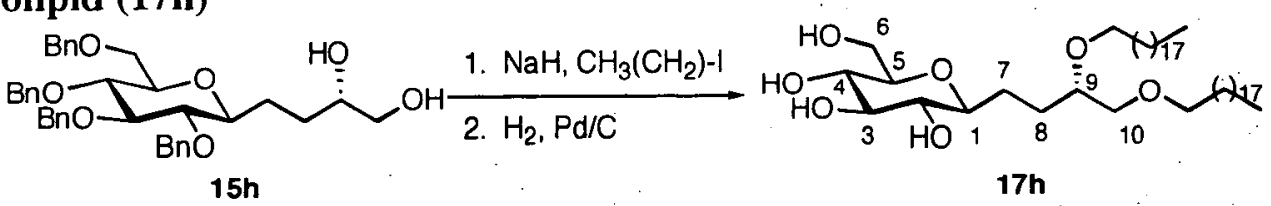

The same procedure for alkylation was followed using: diol $15 \mathrm{~h}(30 \mathrm{mg}, 48.9 \mu \mathrm{mol}), \mathrm{DMF}(2 \mathrm{~mL}), \mathrm{NaH}$ ( $8 \mathrm{mg}, 0.2 \mathrm{mmol}, 60 \%$ dispersion in mineral oil), bromoeicosane (105 $\mathrm{mg}, 0.29 \mathrm{mmol}$ ), and tetrabutylammonium iodide $(2 \mathrm{mg}, 4.9 \mu \mathrm{mol})$. After the usual work-up, flash chromatography over silica gel using 20\% $\mathrm{Et}_{2} \mathrm{O}$-hexanes; $1 \mathrm{H} \mathrm{NMR}\left(500 \mathrm{MHz}, \mathrm{CDCl}_{3}\right.$ ); HRMS (FAB) calcd for $\mathrm{C}_{78} \mathrm{H}_{124} \mathrm{O}_{7} \mathrm{Na}(\mathrm{M})^{+}$ 1 195.9239, found 1195.9268 . The product was then taken directly to the next step and the general method of hydrogenolysis was followed using: $16 \mathrm{~h}(12 \mathrm{mg}, 10.0 \mu \mathrm{mol})$ in EtOAc:MeOH $(1: 1,5 \mathrm{~mL})$ was added $\mathrm{Pd} / \mathrm{C}(10 \mathrm{mg})$. Flash chromatography of the residue over silica gel using $5 \rightarrow 10 \% \mathrm{MeOH}-\mathrm{CH}_{2} \mathrm{Cl}_{2}$ gave $17 \mathrm{~h}(6.5 \mathrm{mg}, 76 \%)$ as a pure $\left(\mathrm{R}_{\mathrm{f}}=0.27\right.$, TLC, silica, $10 \% \mathrm{MeOH}-\mathrm{CH}_{2} \mathrm{Cl}_{2} ;{ }^{1} \mathrm{H} \mathrm{NMR}(500 \mathrm{MHz}$, $\left.\left.\mathrm{CDCl}_{3}\right)\right]$ white solid: m.p. $=70-71^{\circ} \mathrm{C} ;[\alpha]_{\mathrm{D}}^{26}=+8.9\left(c=1.00, \mathrm{CH}_{2} \mathrm{Cl}_{2}\right)$; FT-IR (neat) $3426(\mathrm{~b}), 2914,2861$, $1612,1513,1463,1362,1301,1247,1173,1085,1033,820 \mathrm{~cm}^{-1} ;{ }^{1} \mathrm{H}$ NMR $\left(500 \mathrm{MHz}, \mathrm{CDCl}_{3}\right) \delta 3.76(\mathrm{dd}$, $1 \mathrm{H}, J=12.0,3.0 \mathrm{~Hz}, H-6$ ), 3.63 (dd, $1 \mathrm{H}, J=12.0,5.0 \mathrm{~Hz}, H-6$ ), 3.54 (ddd, $1 \mathrm{H}, J=9.5,7.5 \mathrm{~Hz}, 7.5 \mathrm{~Hz}$, $H-9), 3.43-3.35\left(\mathrm{~m}, 4 \mathrm{H}, 2 \times H-10, \mathrm{OCH}_{2}\right), 3.35-3.28\left(\mathrm{~m}, 5 \mathrm{H}, \mathrm{H}-2, \mathrm{H}-3, \mathrm{H}-4, \mathrm{OCH}_{2}\right), 3.20-3.13(\mathrm{~m}, 1 \mathrm{H}$, $H-5), 3.10(\mathrm{dd}, 1 \mathrm{H}, J=9.0,9.0 \mathrm{~Hz}, H-1), 2.27-2.22(\mathrm{~m}, 1 \mathrm{H}, H-7), 1.85-1.78(\mathrm{~m}, 1 \mathrm{H}, H-8), 1.63-1.44(\mathrm{~m}$, $\left.6 \mathrm{H}, \mathrm{H}-7, \mathrm{H}-8,2 \times \mathrm{CH}_{2}\right), 1.26-1.18\left(\mathrm{bs}, 68 \mathrm{H}, 34 \times \mathrm{CH}_{2}\right), 0.82\left(\mathrm{t}, 6 \mathrm{H}, \mathrm{J}=7.0 \mathrm{~Hz}, 2 \times \mathrm{CH}_{3}\right) ;{ }^{13} \mathrm{C} \mathrm{NMR}(125$ $\left.\mathrm{MHz}, \mathrm{CDCl}_{3}\right) \delta 79.1,78.9,78.6,78.3 ; 73.2,73.2,71.6,70.6,70.5,62.2,31.8,30.0,29.6,29.5,29.5,29.4$, 29.4, 29.2, 27.3, 26.0, 22.6, 14.0; HRMS (FAB) calcd for $\mathrm{C}_{50} \mathrm{H}_{100} \mathrm{O}_{7} \mathrm{Na}(\mathrm{M})^{+}$835.7361, found 835.7396.

\section{ENTRY 9}

\section{C-Glycoglycerolipid (17i)}

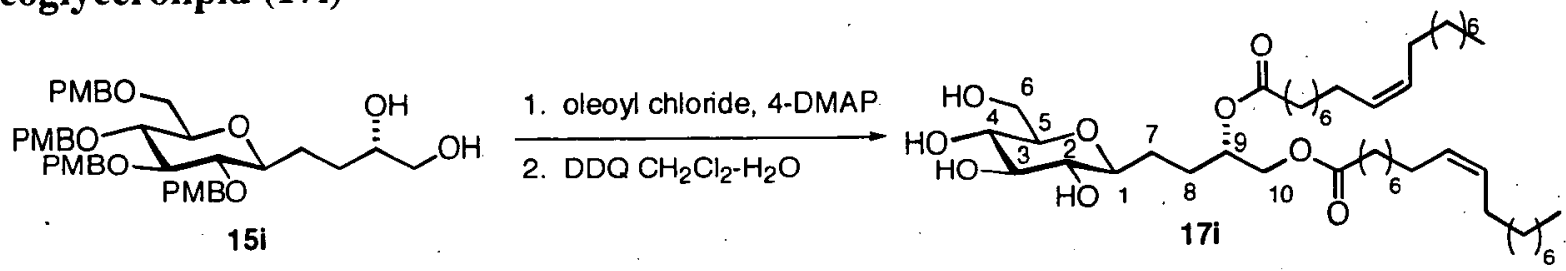

The general method for esterification was followed using: diol $15 \mathbf{i}$ ( $40 \mathrm{mg}, 0.055 \mathrm{mmol}$ ), oleoyl chloride $(0.1 \mathrm{~mL}, 0.33 \mathrm{mmol})$, and 4-DMAP $(3 \mathrm{mg}, 0.027 \mathrm{mmol})$ in $\mathrm{CH}_{2} \mathrm{Cl}_{2}(2 \mathrm{~mL})$. Flash chromatography of the residue over silica using 15-30\% EtOAc-hexanes gave $16 \mathbf{i}(48 \mathrm{mg}, 80 \%)$ as a pure $\left(R_{f}=0.35\right.$, TLC, silica, $30 \%$ EtOAc-hexanes; ' $\mathrm{H}$ NMR (500 MHz, CDCl $)$; HRMS (FAB) calcd for $\mathrm{C}_{78} \mathrm{H}_{116} \mathrm{O}_{13} \mathrm{Na}(\mathrm{M}){ }^{+}$ 1283.8308, found 1283.8390) yellow oil. The product was then taken directly to the next step and a 
(C) 2004 American Chemical Society, J. Org. Chem., Postema jo040254t Supporting Info Page 24

solution of $16 \mathrm{i}(40 \mathrm{mg}, 0.032 \mathrm{mmol})$ in $\mathrm{CH}_{2} \mathrm{Cl}_{2}(2.4 \mathrm{~mL})$ and $\mathrm{H}_{2} \mathrm{O}(0.1 \mathrm{~mL})$ was treated with 2,3-dichloro5,6-dicyano-1,4-benzoquinone (DDQ) $(29 \mathrm{mg}, 0.127 \mathrm{mmol})$. The reaction was stirred for $1 \mathrm{~h}$ at ambient temperature, diluted with $\mathrm{H}_{2} \mathrm{O}(2 \mathrm{~mL})$ and $\mathrm{CH}_{2} \mathrm{Cl}_{2}(2 \mathrm{~mL})$, the aqueous phase was extracted with $\mathrm{CH}_{2} \mathrm{Cl}_{2}$ $(3 \times 10 \mathrm{~mL})$. The organic extracts were dried with $\mathrm{MgSO}_{4}$, filtered and concentrated in vacuo to give a crude oil. Flash chromatography of the residue over silica using $5 \rightarrow 10 \% \mathrm{CH}_{2} \mathrm{Cl}_{2}-\mathrm{MeOH}$ gave $17 \mathrm{i}$ (18 $\mathrm{mg}, 73 \%)$ as a pure $\left(\mathrm{R}_{\mathrm{f}}=0.22\right.$, TLC, silica, $\left.5 \% \mathrm{CH}_{2} \mathrm{Cl}_{2}-\mathrm{MeOH} ;{ }^{1} \mathrm{H} \mathrm{NMR}\left(400 \mathrm{MHz}, \mathrm{CDCl}_{3}\right)\right)$ white solid: m.p. $=99-100^{\circ} \mathrm{C} ;\left[\alpha b^{26}=-16.2\left(c=1.00, \mathrm{CHCl}_{3}\right) ; \mathrm{FT}-\mathrm{IR}\right.$ (neat) $3390,2924,2854,1738,1464,1378$, $1174,1089,1013,723 \mathrm{~cm}^{-1} ;{ }^{1} \mathrm{H}$ NMR $\left(400 \mathrm{MHz}, \mathrm{CDCl}_{3}\right) 85.34-5.24(\mathrm{~m}, 4 \mathrm{H}, 4 \mathrm{x}=\mathrm{C}-\mathrm{H}), 5.15-5.08(\mathrm{~m}, 1$ $\mathrm{H}, H-9), 4.21$ (dd, $1 \mathrm{H}, J=11.5,2.5 \mathrm{~Hz}, H-10), 3.95$ (dd, $1 \mathrm{H}, J=12,7.0 \mathrm{~Hz}, H-10), 3.75$ (dd, $1 \mathrm{H}, J=$ $12.0,2.4 \mathrm{~Hz}, H-6), 3.67$ (dd, $1 \mathrm{H}, J=12,4.8 \mathrm{~Hz}, H-6), 3.38-3.29$ (m, $1 \mathrm{H}, H-4), 3.20-3.12$ (m, $2 \mathrm{H}, H-3$, $H-5$ ), 3.09 (dd; $1 \mathrm{H}, J=6.0,6.0 \mathrm{~Hz}, H-2$ ), 3.07 (dd, $1 \mathrm{H}, J=7.2,7.2 \mathrm{~Hz}, \mathrm{H}-1), 3.04-2.70$ (m, $4 \mathrm{H}, 4 \mathrm{x}$ $\mathrm{OH}), 2.28-2.22\left(\mathrm{~m}, 4 \mathrm{H}, 2 \times \mathrm{CH}_{2}\right), 1.99-1.92\left(\mathrm{~m}, 8 \mathrm{H}, 4 \times \mathrm{CH}_{2}\right), 1.89-1.78(\mathrm{~m}, 1 \mathrm{H}, \mathrm{H}-7), 1.78-1.68(\mathrm{~m}, 1$ $\mathrm{H}, H-8), 1.67-1.59(\mathrm{~m}, 1 \mathrm{H}, H-8), 1.61-1.50\left(\mathrm{~m}, 4 \mathrm{H}, 2 \times \mathrm{CH}_{2}\right), 1.50-1.38(\mathrm{~m}, 1 \mathrm{H}, H-7), 1.32-1.19(\mathrm{~m}, 36$ $\left.\mathrm{H}, 18 \times \mathrm{CH}_{2}\right), 0.83\left(\mathrm{t}, 6 \mathrm{H}, J=5.6 \mathrm{~Hz}, 2 \times \mathrm{CH}_{3}\right) ;{ }^{13} \mathrm{C} \mathrm{NMR}\left(125 \mathrm{MHz}, \mathrm{CDCl}_{3}\right) \delta 173.9,173.8,129.9$, $129.6,79.2,78.7,78.3,73.3,71.2,70.2,64.9,62.0,45.9,34.3,34.0,31.8,29.6,29.6,29.2,29.1,29.0$, $29.0,27.1,27.1,26.7,26.5,24.9,24.7,22.6,14.0$; HRMS (FAB) calcd for $\mathrm{C}_{46} \mathrm{H}_{84} \mathrm{O}_{9} \mathrm{Na}(\mathrm{M})^{+} 803.6008$, found 803.6077 .

\section{ENTRY 10}

See experimental section in paper.

\section{ENTRY 11}

\section{C-Glycoglycerolipid (17k)}

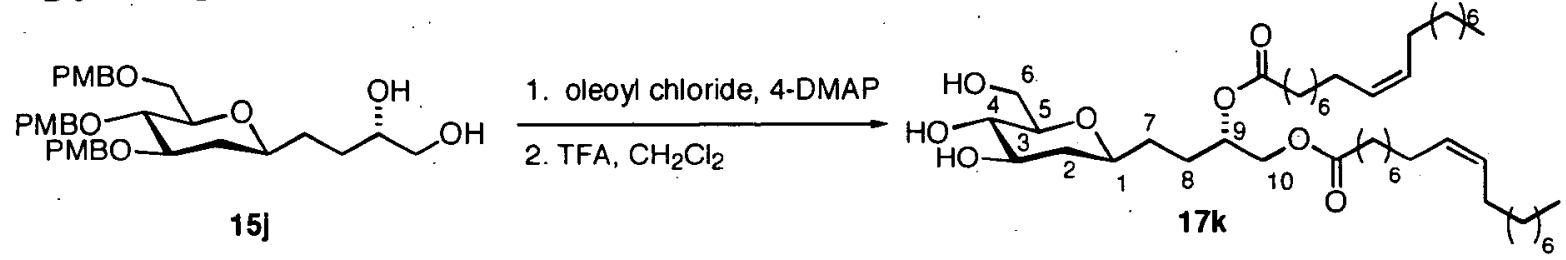

The general method for esterification was followed using: diol $15 \mathrm{j}$ ( $26 \mathrm{mg}, 0.044 \mathrm{mmol})$, oleoyl chloride $(86 \mu \mathrm{L}, 0.26 \mathrm{mmol})$, and 4-DMAP $(3 \mathrm{mg}, 0.022 \mathrm{mmol})$ in pyridine $(1.5 \mathrm{~mL})$. Flash chromatography of the residue over silica using $25 \rightarrow 30 \%$ EtOAc-hexanes gave $16 \mathrm{k}(41 \mathrm{mg}, 85 \%)$ as a pure $\left(\mathrm{R}_{\mathrm{f}}=0.52, \mathrm{TLC}\right.$, silica, $30 \%$ EtOAc-hexanes; ' $\mathrm{H}$ NMR $\left(500 \mathrm{MHz}, \mathrm{CDCl}_{3}\right)$ ) yellow oil. The product was then taken directly to the next step and TFA ( 5 drops) was added to a solution of $16 \mathrm{k}(25 \mathrm{mg}, 0.022 \mathrm{mmol}$ ) in $\mathrm{CH}_{2} \mathrm{Cl}_{2}(2.0 \mathrm{~mL})$ and the reaction was stirred for $1 \mathrm{~h}$ at ambient temperature. At this point TLC analysis (silica, first $30 \%$ EtOAc-hexanes then $5 \% \mathrm{MeOH}-\mathrm{CH}_{2} \mathrm{Cl}_{2}$ ) indicated the reaction was almost complete and upon concentration in vacuo the reaction went to completion. Flash chromatography of the residue over silica gel using $3 \rightarrow 5 \% \mathrm{MeOH}-\mathrm{CH}_{2} \mathrm{Cl}_{2}$ gave $17 \mathrm{k}(14.5 \mathrm{mg}, 85 \%)$ as a pure $\left(\mathrm{R}_{\mathrm{f}}=0.33\right.$, TLC, silica, $5 \%$ MeOH- $\mathrm{CH}_{2} \mathrm{Cl}_{2}$; ${ }^{1} \mathrm{H}$ NMR $\left.\left(400 \mathrm{MHz}, \mathrm{CDCl}_{3}\right)\right)$ clear oil: $[\alpha]^{26}=+4.9\left(c=1.00, \mathrm{CH}_{2} \mathrm{Cl}_{2}\right) ; \mathrm{FT}$-IR (neat) 3390 (b), 2921, 2852, 1736, 1463, 1376, 1245, 1168, 1088, 1052, $722 \mathrm{~cm}^{-1}$; ${ }^{1} \mathrm{H}$ NMR (400 MHz, $\left.\mathrm{CDCl}_{3}\right) \delta$ 5.32-5.20 (m, $4 \mathrm{H}, 4$ x C=C- $H$ ), 5.10-5.00 (m, $1 \mathrm{H}, H-9), 4.25$ (dd, $1 \mathrm{H}, J=11.6,3.2 \mathrm{~Hz}, H-10), 3.99$ (dd, $1 \mathrm{H}, J=12.4,6.8 \mathrm{~Hz}, H-10), 3.71$ (dd, $1 \mathrm{H}, J=12.0,4.5 \mathrm{~Hz}, H-6), 3.44-3.35$ (m, $2 \mathrm{H}, H-3, H-4), 3.24-$ $3.18(\mathrm{~m}, 2 \mathrm{H}, H-1, H-5), 3.13(\mathrm{dd}, 1 \mathrm{H}, J=8.5,8.5 \mathrm{~Hz}, H-2), 2.30-2.24\left(\mathrm{~m}, 4 \mathrm{H}, \mathrm{C}(=\mathrm{O}) \mathrm{CH}_{2}-\right), 2.21-2.13$ (m, $4 \mathrm{H}, 4 \times \mathrm{OH}), 1.90-1.82(\mathrm{~m}, 1 \mathrm{H}, \mathrm{H}-7), 1.80-1.71(\mathrm{~m}, 1 \mathrm{H}, \mathrm{H}-8), 1.70-1.62(\mathrm{~m}, 1 \mathrm{H}, \mathrm{H}-8), 1.62-1.52$ $\left(\mathrm{m}, 4 \mathrm{H}, 2 \times \mathrm{CH}_{2}\right), 1.52-1.43(\mathrm{~m}, 1 \mathrm{H}, H-7), 1.30-1.21\left(\mathrm{~m}, 92 \mathrm{H}, 46 \times \mathrm{CH}_{2}\right), 0.85(\mathrm{t}, 6 \mathrm{H}, J=7.0 \mathrm{~Hz}, 2 \mathrm{x}$ 
(C) 2004 American Chemical Society, J. Org. Chem., Postema jo040254t Supporting Info Page 25

Postema, Piper, Betts, Valeriote and Pietraszkewicz RCM-Based Synthesis of a Variety of $\beta$-C-Glycosides and their In Vitro Anti-Solid Tumor Activity

$\left.\mathrm{CH}_{3}\right) ;{ }^{13} \mathrm{C} \mathrm{NMR}\left(125 \mathrm{MHz}, \mathrm{CDCl}_{3}\right) \delta 173.6,173.6,130.0,130.0,129.7,129.7,78.9,77.2,75.0,72.8$, $72.7,70.8,64.9,62.5,55.2,38.6,34.4,34.1,31.9,30.5,29.7,29.7,29.5,29.3,29.2,29.1,29.1,27.2,27.2$, 26.8, 25.0, 24.8, 22.7, 14.1; HRMS (FAB) calcd for $\mathrm{C}_{46} \mathrm{H}_{84} \mathrm{O}_{8} \mathrm{Na}(\mathrm{M})^{+}$787.6058, found 787.6573.

\section{O-Glycoside Examples}

The known corresponding $\beta$-O-glycoglycerolipid 171 was prepared in a straightforward manner from the known tetraacetate $\mathbf{1 8}^{15}$ as delineated in Scheme $S-1$.

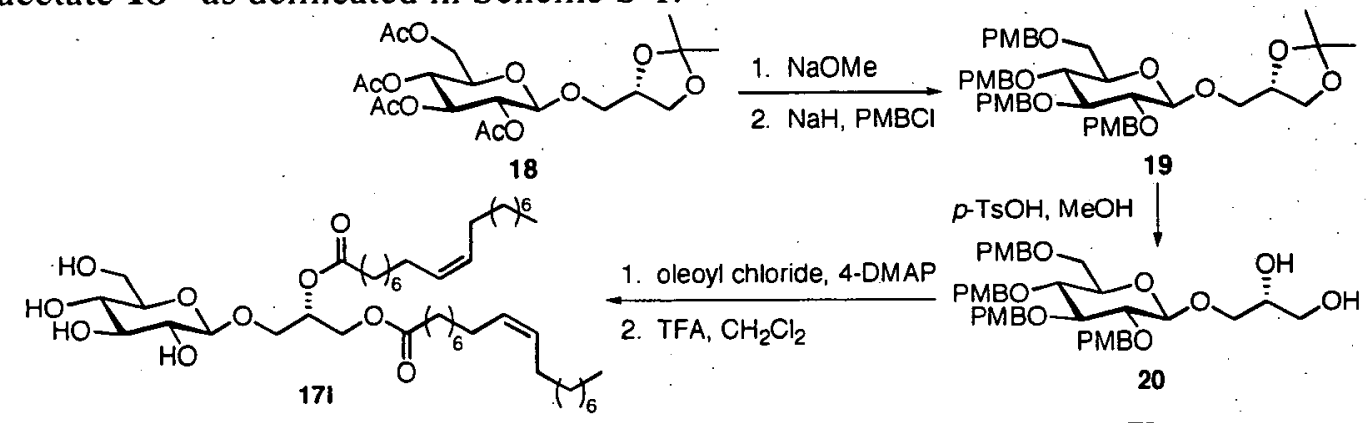

Scheme S-1. Preparation of $\beta$-O-glycoglycerolipid 171

PMB-Acetonide (19)
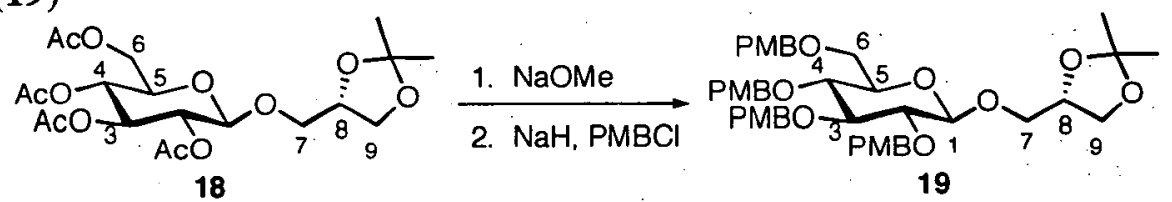

A catalytic amount of pre-washed $\mathrm{NaH}(\sim 5 \mathrm{mg})$ was added in one portion to a methanol $(50 \mathrm{~mL})$ solution of tetratacetate 18 (448 $\mathrm{mg}, 0.97 \mathrm{mmol})$. The resulting solution was stirred for $1 \mathrm{~h}$ at ambient temperature and the reaction was then quenched by the addition of acetic acid $(0.5 \mathrm{~mL})$ and the resulting solution concentrated in vacuo and then azeotroped with benzene $(20 \mathrm{~mL})$. The resulting oil was pumped under vacuum $(0.5 \mathrm{~mm} \mathrm{Hg})$ for 6 hours and then dissolved in DMF $(20 \mathrm{~mL})$ and the resulting solution cooled to $0{ }^{\circ} \mathrm{C}$ and $\mathrm{PMBCl}(4.85 \mathrm{~mL}, 4.85 \mathrm{mmol}, 5 \mathrm{eq})$, TBAI (36 mg, $\left.97 \mu \mathrm{mol}, 0.1 \mathrm{eq}\right)$ and sodium hydride (195 $\mathrm{mg}, 4.85 \mathrm{mmol}, 5 \mathrm{eq})$ were added sequentially to the cool solution. The resulting mixture was then allowed to warm to ambient temperature over $1 \mathrm{~h}$ and then heated to $80^{\circ} \mathrm{C}$ for 12 hours. The solution was cooled and carefully quenched by the addition of $\mathrm{MeOH}(10 \mathrm{~mL})$. The solution was concentrated in vacuo and the residue dissolved in ether $(100 \mathrm{~mL})$. The ethereal fraction was washed with saturated ammonium chloride $(1 \times 30 \mathrm{~mL})$, brine $(1 \times 30 \mathrm{~mL})$, dried and concentrated. Flash chromatography of the residue over silica using $20 \rightarrow 40 \%$ ether-hexanes gave $19(641 \mathrm{mg}, 85 \%$ over 2 steps $)$ as a pure $\left({ }^{1} \mathrm{H}\right.$ NMR (500 MHz, $\mathrm{CDCl}_{3}$ ) oil: $[\alpha]_{D}^{26}=6.91\left(c=1.00, \mathrm{CHCl}_{3}\right)$; FT-IR (neat) 2916, 2848, 1612, 1514, 1472, $1462,1248,1173,1071,1036,820,720 \mathrm{~cm}^{-1}$; 'H NMR (500 MHz, CDCl $)$ 8 7.26-7.18 (m, $\left.7 \mathrm{H}, \mathrm{ArH}\right)$, 7.02-7.00 (m, $2 \mathrm{H}, \mathrm{ArH}), 6.85-6.76(\mathrm{~m}, 7 \mathrm{H}, \mathrm{ArH}), 4.81\left(\mathrm{~d}, 1 \mathrm{H}, J=10.5 \mathrm{~Hz}, \mathrm{OCH}_{2} \mathrm{Ar}\right), 4.80(\mathrm{~d}, 1 \mathrm{H}, J=$ $\left.11 \mathrm{~Hz}, \mathrm{OCH}_{2} \mathrm{Ar}\right), 4.69\left(\mathrm{~d}, 1 \mathrm{H}, J=10 \mathrm{~Hz}, \mathrm{OCH}_{2} \mathrm{Ar}\right), 4.68\left(\mathrm{~d}, 1 \mathrm{H}, J=10.5 \mathrm{~Hz}, \mathrm{OCH}_{2} \mathrm{Ar}\right), 4.61(\mathrm{~d}, 1 \mathrm{H}, J=$ $\left.10.5 \mathrm{~Hz}, \mathrm{OCH}_{2} \mathrm{Ar}\right), 4.52$ (d, $1 \mathrm{H}, J=12 \mathrm{~Hz}, \mathrm{OCH}_{2} \mathrm{Ar}$ ), 4.44 (d, $1 \mathrm{H}, J=12 \mathrm{~Hz}, \mathrm{OCH}_{2} \mathrm{Ar}$ ), 4.38 (d, $1 \mathrm{H}, J=$ $10.5 \mathrm{~Hz}, \mathrm{OCH} \mathrm{H}_{2} \mathrm{Ar}$ ), 4.36 (d, $1 \mathrm{H}, J=8.0 \mathrm{~Hz}, H-1$ ), 4.32 (dddd, $1 \mathrm{H}, J=4.0,4.0,4.0,4.0 \mathrm{~Hz}, H-8$ ), 4.05 (dd, $1 \mathrm{H}, J=8.0,6.0 \mathrm{~Hz}, H-7$ or $H-9), 3.98(\mathrm{dd}, 1 \mathrm{H}, J=10,4.5 \mathrm{~Hz}, H-7$ or $H-9$ ), 3.84 (dd, $1 \mathrm{H}, J=8.5$, $6.0 \mathrm{~Hz}, H-7$ or $H-9), 3.78\left(\mathrm{~s}, 3 \mathrm{H}, \mathrm{OCH}_{3}\right), 3.77$ (s, $\left.3 \mathrm{H}, \mathrm{OCH}_{3}\right), 3.76\left(\mathrm{~s}, 6 \mathrm{H}, 2 \times \mathrm{OCH}_{3}\right), 3.64(\mathrm{dd}, 1 \mathrm{H}, J=$ $10.5,1.5 \mathrm{~Hz}, H-6), 3.60-3.54$ (m, $2 \mathrm{H}, H-6, H-7$ or $H-9), 3.54$ (dd, $1 \mathrm{H}, J=8.5,8.5 \mathrm{~Hz}, H-4), 3.48$ (dd, 1

15 van Boeckel, C. A. A.; Visser, G. M.; van Boom, J. H. Tetrahedron 1985, 41, 4557-4565. 
(C) 2004 American Chemical Society, J. Org. Chem., Postema jo040254t Supporting Info Page 26

Postema, Piper, Betts, Valeriote and Pietraszkewicz RCM-Based Synthesis of a Variety of $\beta$-C-Glycosides and their In Vitro Anti-Solid Tumor Activity

$\mathrm{H}, J=9.5,9.5 \mathrm{~Hz}, H-3), 3.38(\mathrm{dd}, 1 \mathrm{H}, J=8.5,8.5 \mathrm{~Hz}, H-2), 3.38-3.35(\mathrm{~m}, 1 \mathrm{H}, H-5), 1.39(\mathrm{~s}, 3 \mathrm{H}$, $\left.\mathrm{C}\left(\mathrm{CH}_{3}\right)_{2}\right), 1.34\left(\mathrm{~s}, 3 \mathrm{H}, \mathrm{C}\left(\mathrm{CH}_{3}\right)_{2}\right) ;{ }^{13} \mathrm{C} \mathrm{NMR}\left(125 \mathrm{MHz}, \mathrm{CDCl}_{3}\right) \delta 130.9,130.6,130.3,130.2,129.8,129.6$, $129.5,113.8,103.9,84.4,81.8,77.5,75.3,74.9,74.6,74.5,74.3,73.1,70.3,68.4,67.0,55.3,26.9,25.4$;

HRMS (FAB) calcd for $\mathrm{C}_{44} \mathrm{H}_{54} \mathrm{O}_{12} \mathrm{Na}(\mathrm{M})^{+} 797.3507$, found 797.3536 .

PMB-Diol (20)

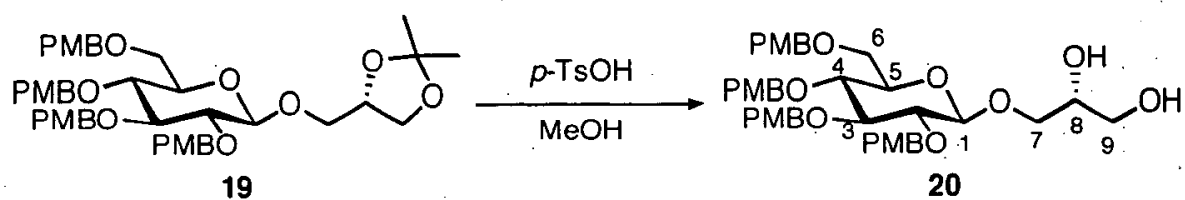

The usual procedure for acetonide deprotection was employed using: $\mathrm{CH}_{2} \mathrm{Cl}_{2}(4 \mathrm{~mL}), \mathrm{MeOH}(4 \mathrm{~mL}), p$ TsOH (15 mg, $0.007 \mathrm{mmol})$ and the acetonide sugar $19(200 \mathrm{mg}, 0.258 \mathrm{mmol})$. After the usual work-up, flash chromatography of the residue over silica using $3 \rightarrow 5 \% \mathrm{MeOH}-\mathrm{CH}_{2} \mathrm{Cl}_{2}$ gave $20(138 \mathrm{mg}, 73 \%)$ as a pure $\left(\mathrm{R}_{\mathrm{f}}=0.23\right.$, TLC, silica, $\left.5 \% \mathrm{CH}_{2} \mathrm{Cl}_{2}-\mathrm{MeOH} ;{ }^{1} \mathrm{H} \mathrm{NMR}\left(500 \mathrm{MHz}, \mathrm{CDCl}_{3}\right)\right)$ white solid: $\left[\alpha \alpha_{b}^{26}=+21.2\right.$ $\left(c=1.00, \mathrm{CHCl}_{3}\right.$ ); FT-IR (neat) 3450 (br), 2916, 2851, 1612, 1513, 1463, 1302, 1248, 1173, 1068, 1034, $820 \mathrm{~cm}^{-1} ;{ }^{\prime} \mathrm{H}$ NMR $\left(500 \mathrm{MHz}, \mathrm{CDCl}_{3}\right) \delta$ 7.28-7.21 (m, $\left.6 \mathrm{H}, \mathrm{ArH}\right), 7.05$ (app d, $2 \mathrm{H}, J=8.0 \mathrm{~Hz}, \mathrm{Ar} H$ ), 6.89-6.80 (m, $8 \mathrm{H}, \mathrm{ArH}), 4.84\left(\mathrm{~d}, 1 \mathrm{H}, J=11 \mathrm{~Hz}, \mathrm{OCH}_{2} \mathrm{Ar}\right), 4.79\left(\mathrm{~d}, 1 \mathrm{H}, J=10.5 \mathrm{~Hz}, \mathrm{OCH}_{2} \mathrm{Ar}\right), 4.73(\mathrm{~d}$, $\left.1 \mathrm{H}, J=11.5 \mathrm{~Hz}, \mathrm{OCH}_{2} \mathrm{Ar}\right), 4.71\left(\mathrm{~d}, 1 \mathrm{H}, J=10 \mathrm{~Hz}, \mathrm{OCH} \mathrm{H}_{2} \mathrm{Ar}\right), 4.67\left(\mathrm{~d}, 1 \mathrm{H}, J=10.5 \mathrm{~Hz}, \mathrm{OCH}_{2} \mathrm{Ar}\right), 4.50$ (d, $\left.1 \mathrm{H}, J=11.5 \mathrm{~Hz}, \mathrm{OCH} \mathrm{H}_{2} \mathrm{Ar}\right), 4.45\left(\mathrm{~d}, 1 \mathrm{H}, J=11.5 \mathrm{~Hz}, \mathrm{OCH} \mathrm{Ar}_{2} \mathrm{Ar}, 4.40\left(\mathrm{~d}, 1 \mathrm{H}, J=11.5 \mathrm{~Hz}, \mathrm{OCH}_{2} \mathrm{Ar}\right.\right.$ ), $4.37(\mathrm{~d}, 1 \mathrm{H}, J=8.0 \mathrm{~Hz}, H-1), 3.96(\mathrm{dd}, 1 \mathrm{H}, J=11.5,2.5 \mathrm{~Hz}, \mathrm{OCH}), 3.88(\mathrm{~m}, 1 \mathrm{H}, \mathrm{OCH}), 3.80(\mathrm{~s}, 3 \mathrm{H}$, $\left.\mathrm{OCH}_{3}\right), 3.80\left(\mathrm{~s}, 3 \mathrm{H}, \mathrm{OCH}_{3}\right), 3.79\left(\mathrm{~s}, 3 \mathrm{H}, \mathrm{OCH}_{3}\right), 3.79\left(\mathrm{~s}, 3 \mathrm{H}, \mathrm{OCH}_{3}\right), 3.78-3.73(\mathrm{~m}, 1 \mathrm{H}, \mathrm{OCH}), 3.70-$ $3.61(\mathrm{~m}, 2 \mathrm{H}, 2 \times \mathrm{OCH}), 3.59$ (dd, $1 \mathrm{H}, J=8.5,8.5 \mathrm{~Hz}, H-3), 3.54$ (dd, $1 \mathrm{H}, \mathrm{J}=11,5.5 \mathrm{~Hz}, \mathrm{OCH}), 3.51$ $3.43(\mathrm{~m}, 3 \mathrm{H}, 3 \times \mathrm{OCH}), 3.41(\mathrm{dd}, 1 \mathrm{H}, \mathrm{J}=8.5,8.5 \mathrm{~Hz}, \mathrm{H}-2), 2.30(\mathrm{br} \mathrm{s}, 1 \mathrm{H}, \mathrm{OH}) ;{ }^{13} \mathrm{C} \mathrm{NMR}(125 \mathrm{MHz}$, $\left.\mathrm{CDCl}_{3}\right) \delta 159.3,159.2,159.2,159.2,130.6,130.4,130.0,129.7,129.7,129.5,113.8,113.8,104.4,84.3$, 81.8, 77.5, 75.3, 74.6, 74.4, 74.0, 73.1, 71.2, 68.5, 63.3, 55.2; HRMS (FAB) calcd for $\mathrm{C}_{41} \mathrm{H}_{50} \mathrm{O}_{12} \mathrm{Na}(\mathrm{M})^{+}$ 757.3194, found 757.3167 .

\section{$O$-Glycoside Oleoyl Lipid (17l)}

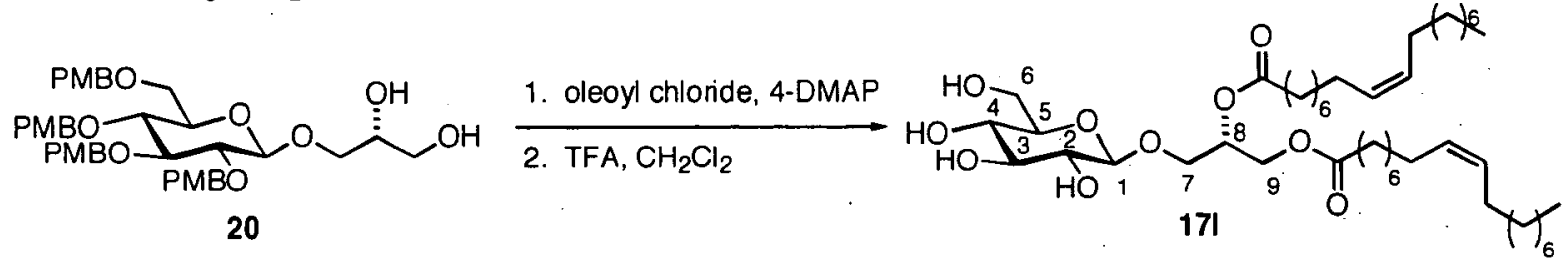

The general method for esterification was followed using: diol $20(60 \mathrm{mg}, 0.082 \mathrm{mmol})$, oleoyl chloride $(162 \mu \mathrm{L}, 0.49 \mathrm{mmol})$, and 4-DMAP $(10 \mathrm{mg})$ in pyridine $(3 \mathrm{~mL})$. Flash chromatography of the residue over silica using $20 \%$ EtOAc-hexanes gave the fully protected $\beta$-O-glycoglycerolipid ( $75 \mathrm{mg}, 73 \%$ ) as a pure $\left(\mathrm{R}_{\mathrm{f}}=0.65\right.$, TLC, silica, $20 \%$ EtOAc-hexanes; ${ }^{1} \mathrm{H}$ NMR ( $\left.500 \mathrm{MHz}, \mathrm{CDCl}_{3}\right)$, HRMS (FAB) calcd for $\mathrm{C}_{77} \mathrm{H}_{114} \mathrm{O}_{14} \mathrm{Na}(\mathrm{M})^{+} 1285.8101$, found 1285.8119$)$ yellow oil. The product was then taken directly to the next step and TFA ( 5 drops) was added to a solution of the fully protected $\beta-O$-glycoglycerolipid $(50 \mathrm{mg}$, $0.039 \mathrm{mmol})$ in $\mathrm{CH}_{2} \mathrm{Cl}_{2}(2.0 \mathrm{~mL})$ and the reaction was stirred for $1 \mathrm{~h}$ at ambient temperature. At this point TLC analysis $\left(20 \% \mathrm{MeOH}-\mathrm{CH}_{2} \mathrm{Cl}_{2}\right)$ indicated the reaction was almost complete and upon concentration in vacuo the reaction went to completion. Flash chromatography of the residue over silica gel using $5 \rightarrow 10 \%$ 
$\mathrm{MeOH}-\mathrm{CH}_{2} \mathrm{Cl}_{2}$ gave $17 \mathrm{I}(22 \mathrm{mg}, 72 \%)$ as a pure $\left(\mathrm{R}_{\mathrm{f}}=0.35\right.$, TLC, silica, $10 \% \mathrm{MeOH}-\mathrm{CH}_{2} \mathrm{Cl}_{2} ;{ }^{1} \mathrm{H} \mathrm{NMR}$ $\left(400 \mathrm{MHz}, \mathrm{CDCl}_{3}\right)$ ) clear oil whose spectral and physical data matched that from the literature. ${ }^{16}$

${ }^{16}$ (a) van Boeckel, C. A. A.; Visser, G. M.; van Boom, J. H. Tetrahedron 1985, 41, 4557-4565. (b) Shirahashi, H.; Murakami, N.; Watanabe, M.; Nagatsu, A.; Sakakibara, J.; Tokuda, H.; Nishino, H.; Iwashima, A. Chem. Pharm. Bull. 1993, 41, 16641666. (c) Nagatsu, A.; Watanabe, M.; Ikemoto, K.; Hashimoto, M.; Murakami, N.; Sakakibara, J.; Tokuda, H.; Nishino, H.; Iwashima, A.; Yazawa, K. Bioorg. Med. Chem. Lett. 1994, 4, 1619-1622. 
(C) 2004 American Chemical Society, J. Org. Chem., Postema jo040254t Supporting Info Page 28

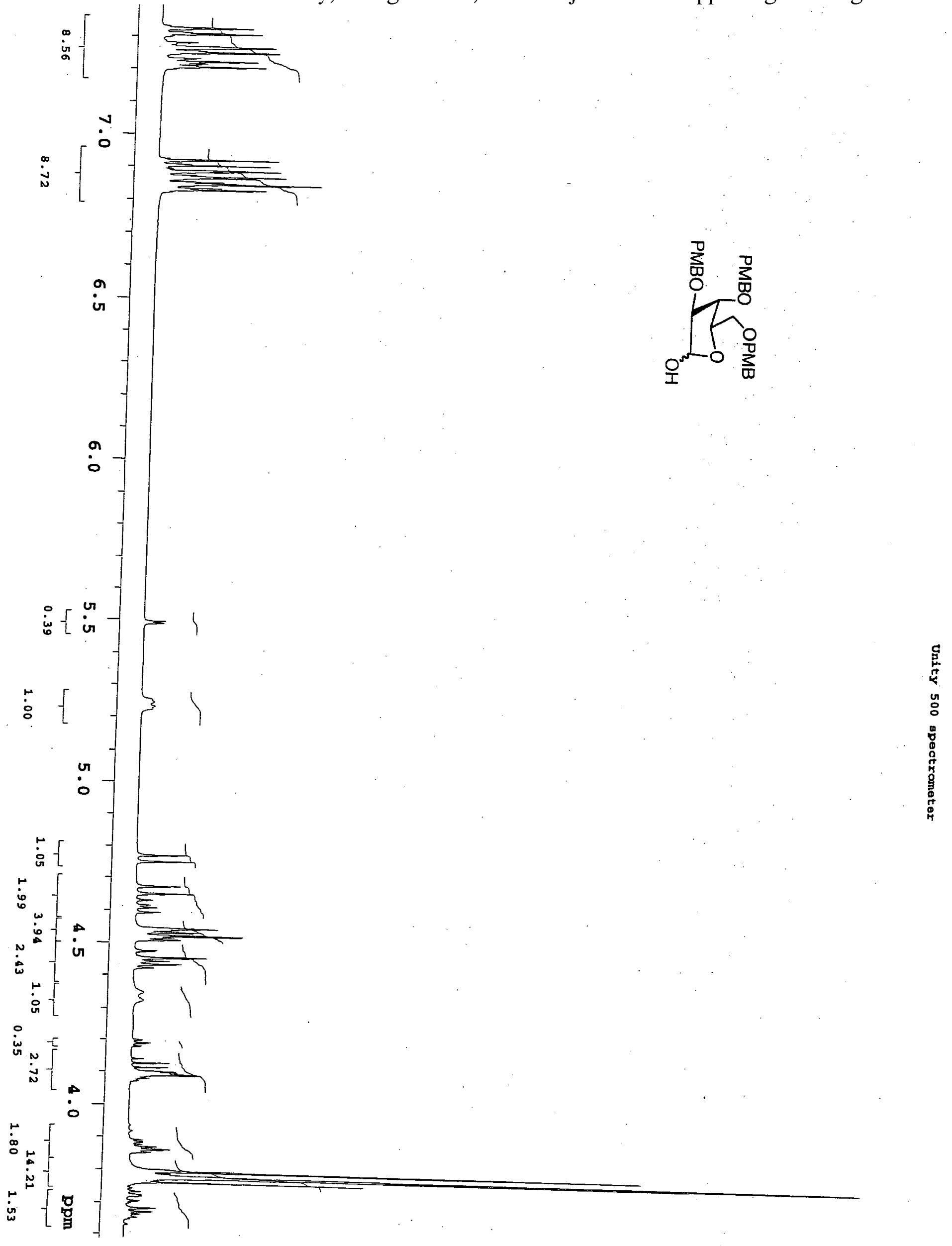


(C) 2004 American Chemical Society, J. Org. Chem., Postema jo040254t Supporting Info Page 29

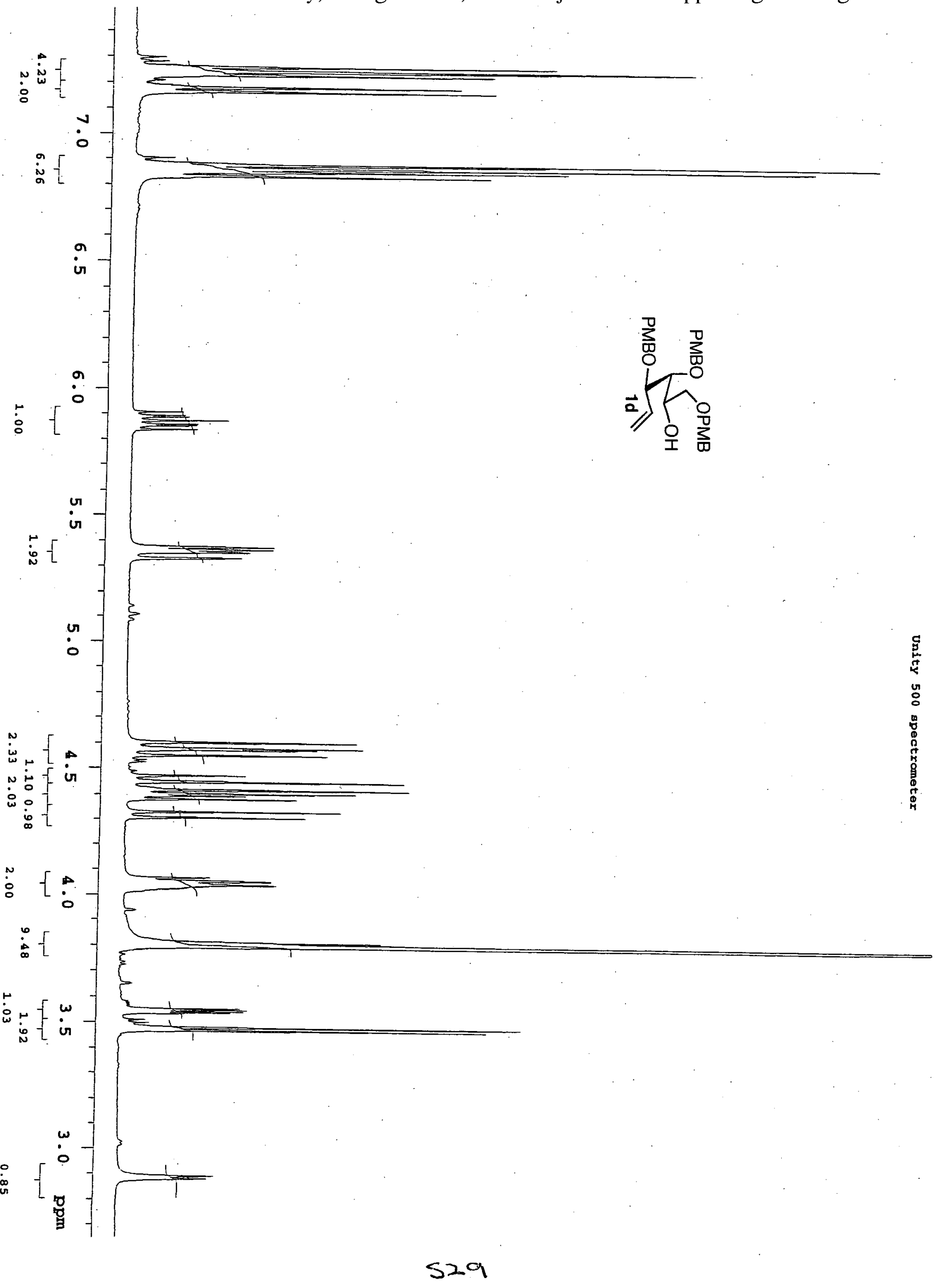


(C) 2004 American Chemical Society, J. Org. Chem., Postema jo040254t Supporting Info Page 30

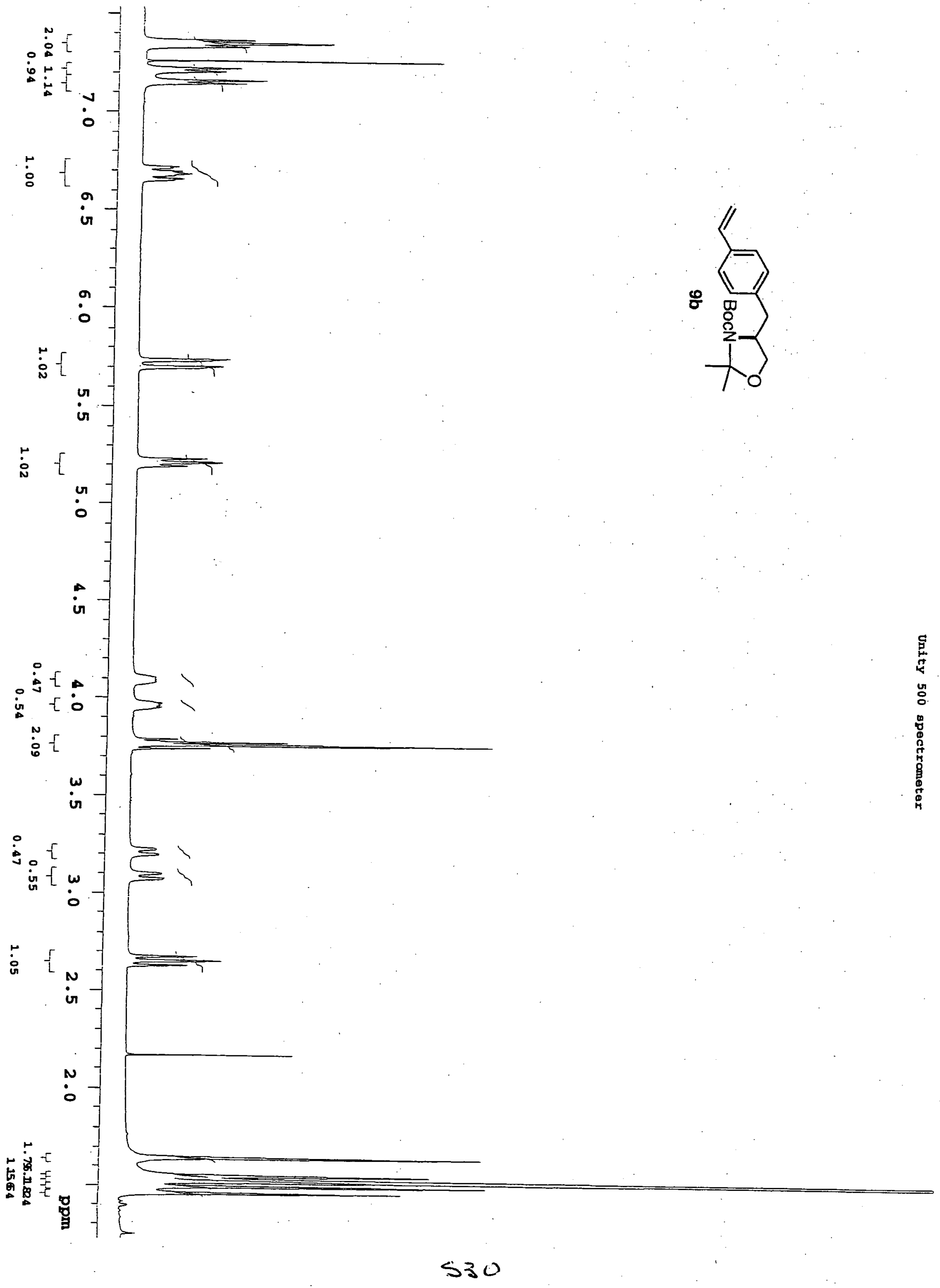


(C) 2004 American Chemical Society, J. Org. Chem., Postema jo040254t Supporting Info Page 31

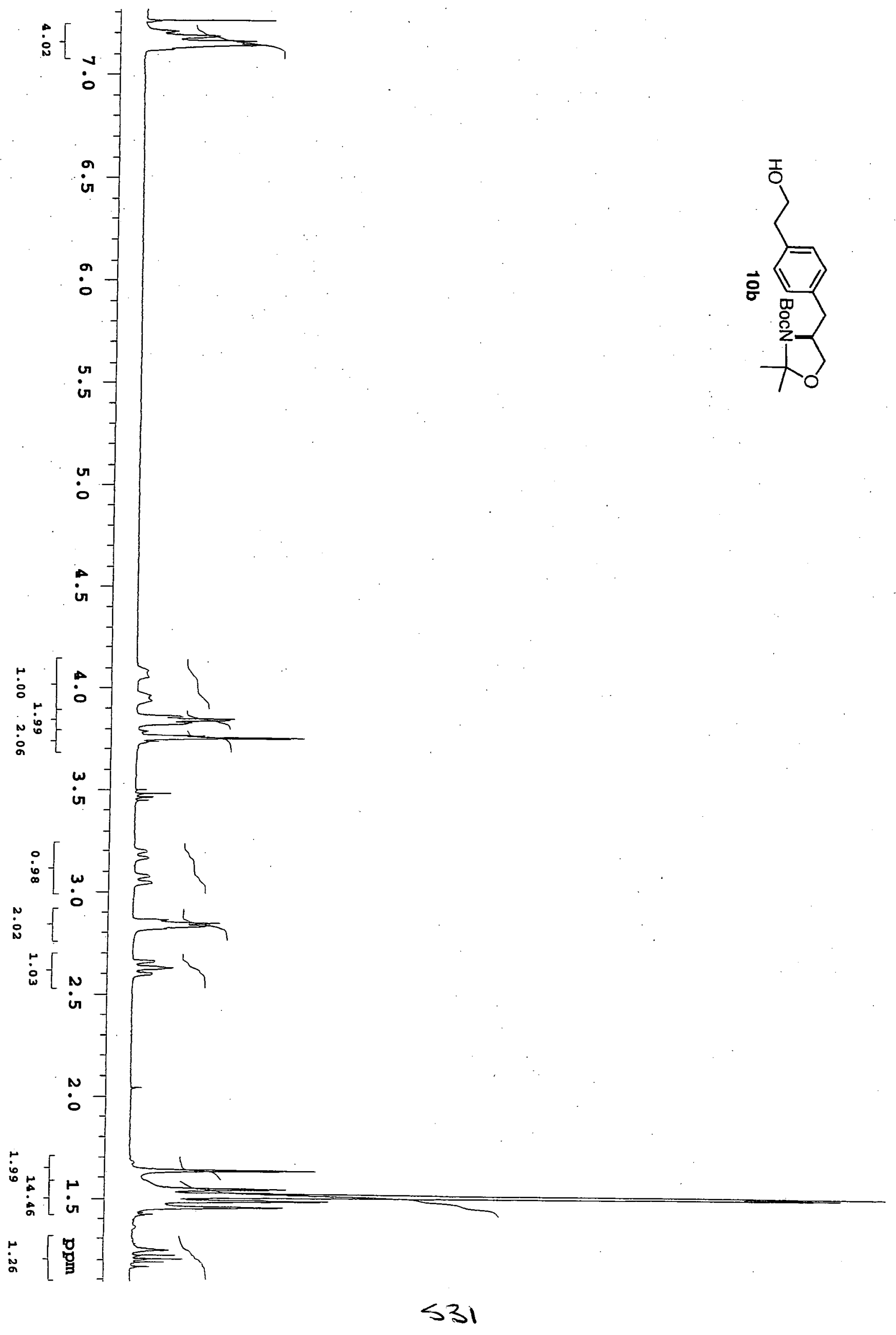


(C) 2004 American Chemical Society, J. Org. Chem., Postema jo040254t Supporting Info Page 32

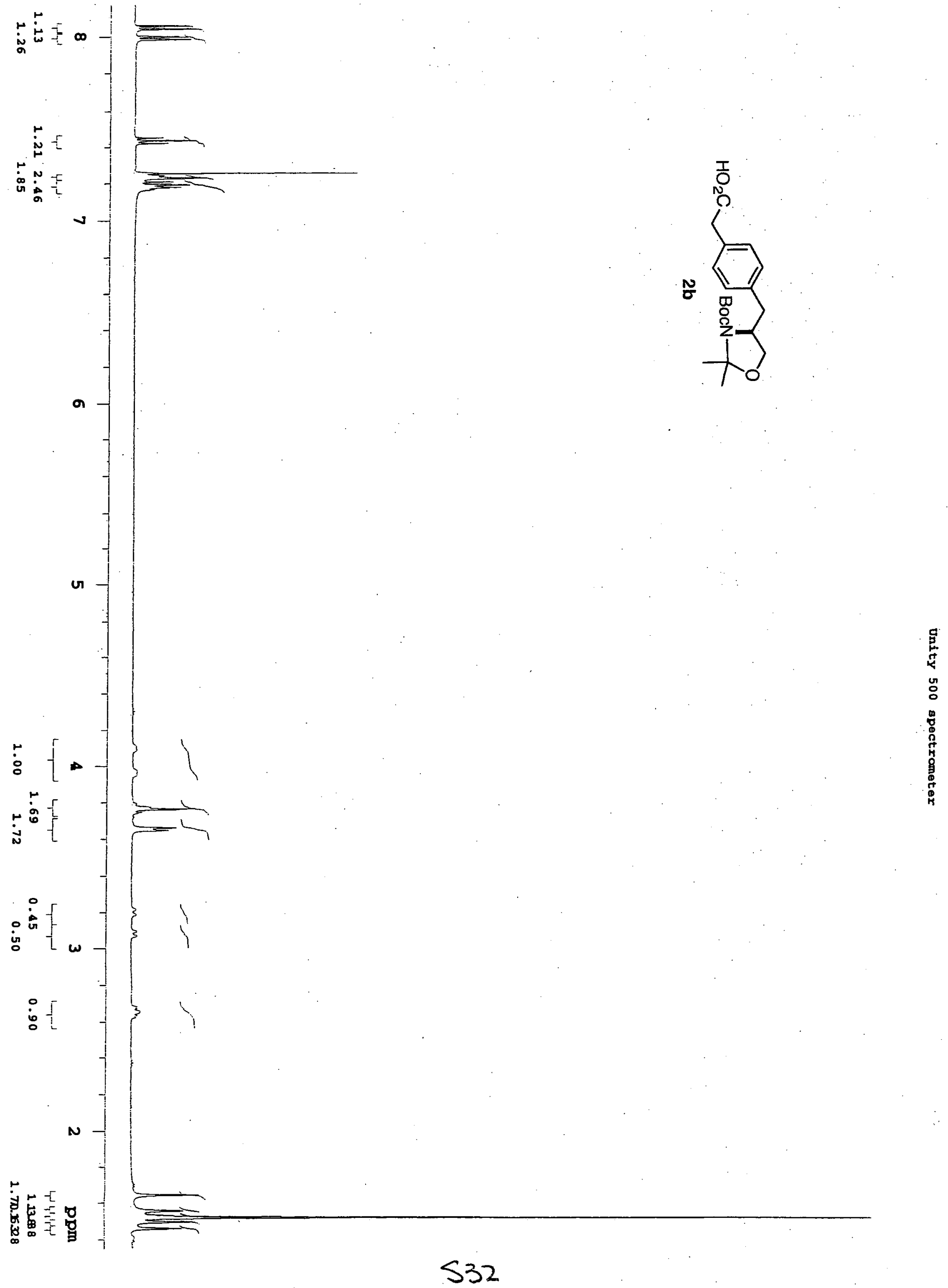


(C) 2004 American Chemical Society, J. Org. Chem., Postema jo040254t Supporting Info Page 33

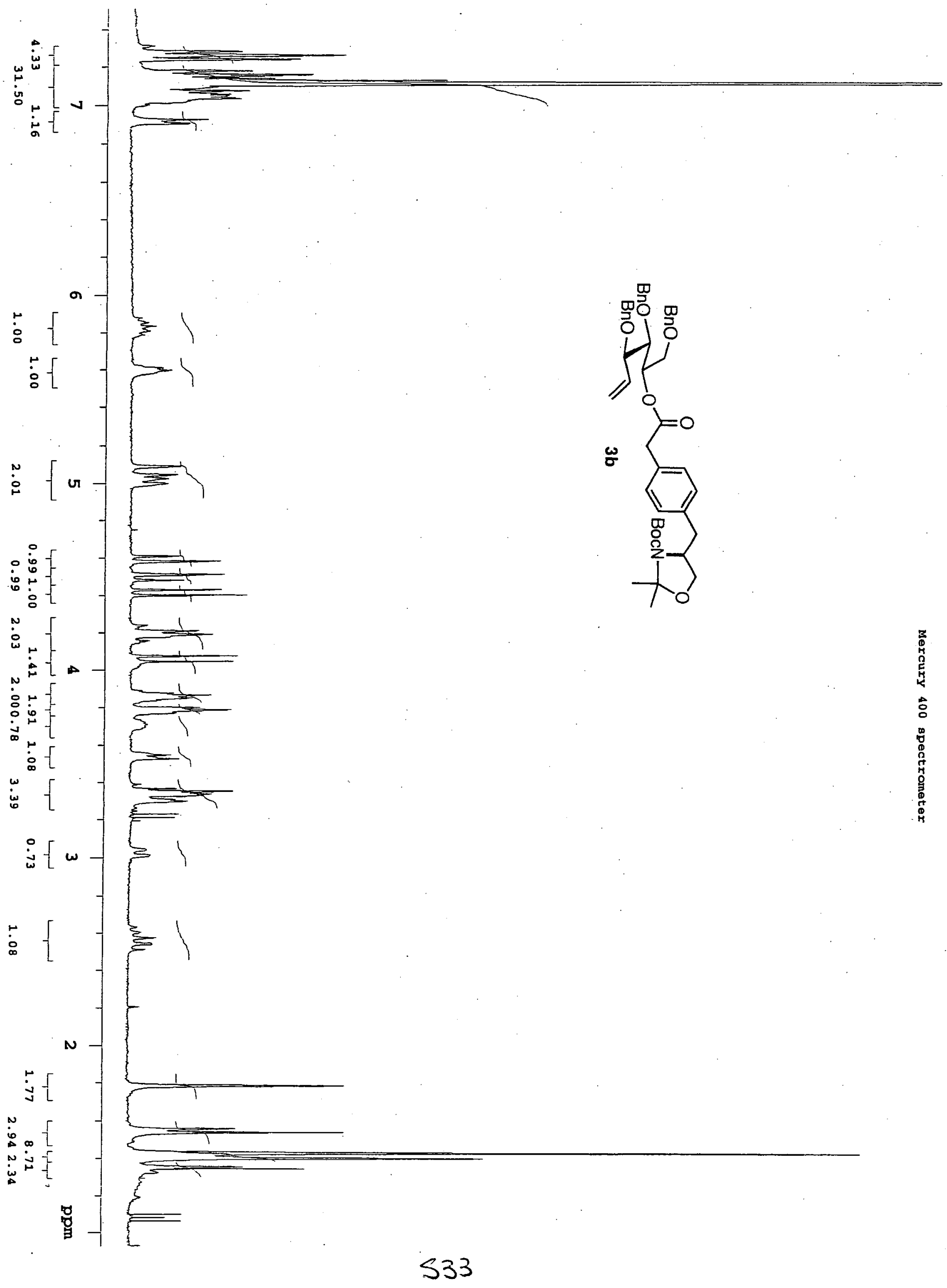


(C) 2004 American Chemical Society, J. Org. Chem., Postema jo040254t Supporting Info Page 34

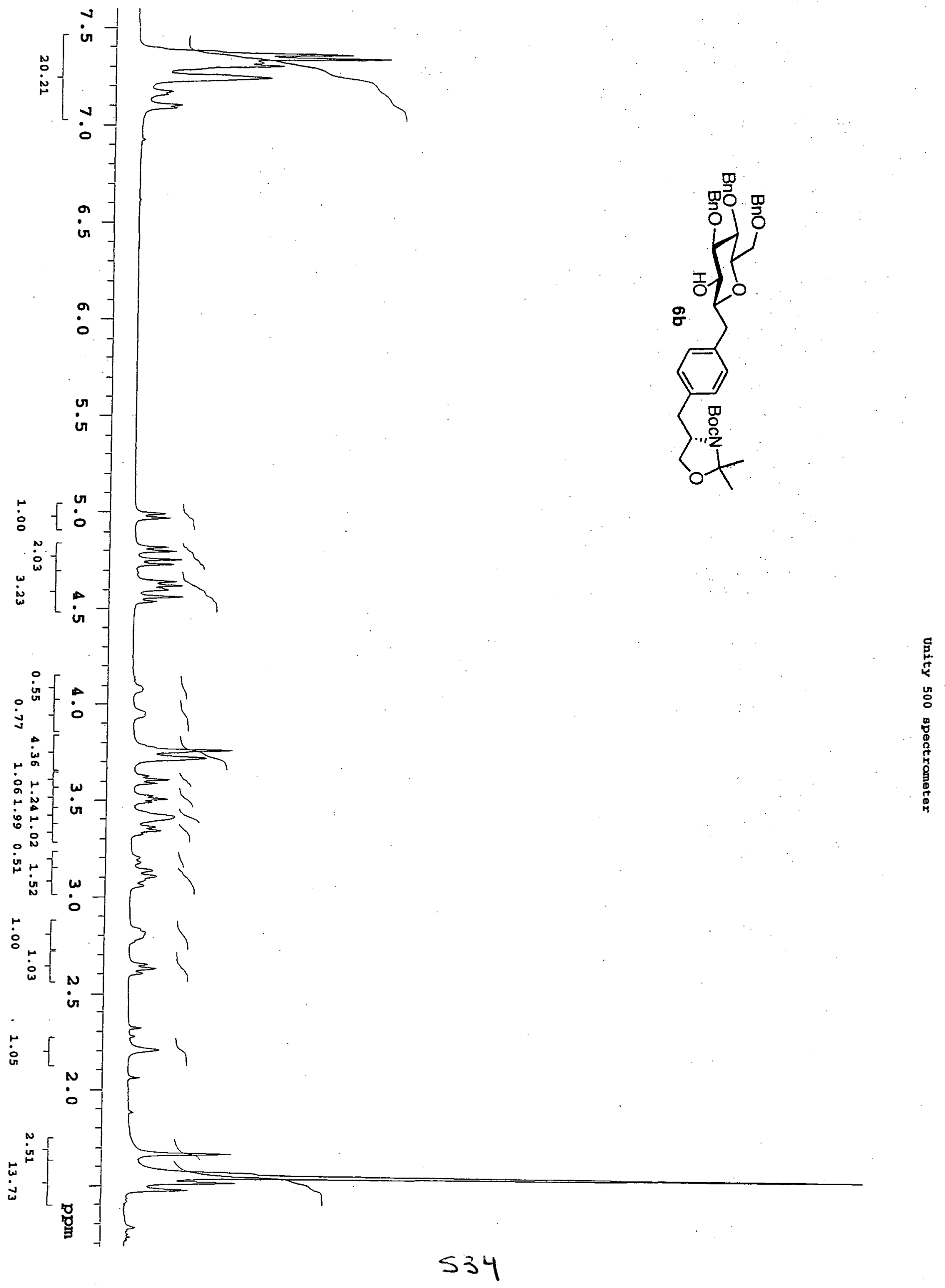


(C) 2004 American Chemical Society, J. Org. Chem., Postema jo040254t Supporting Info Page 35

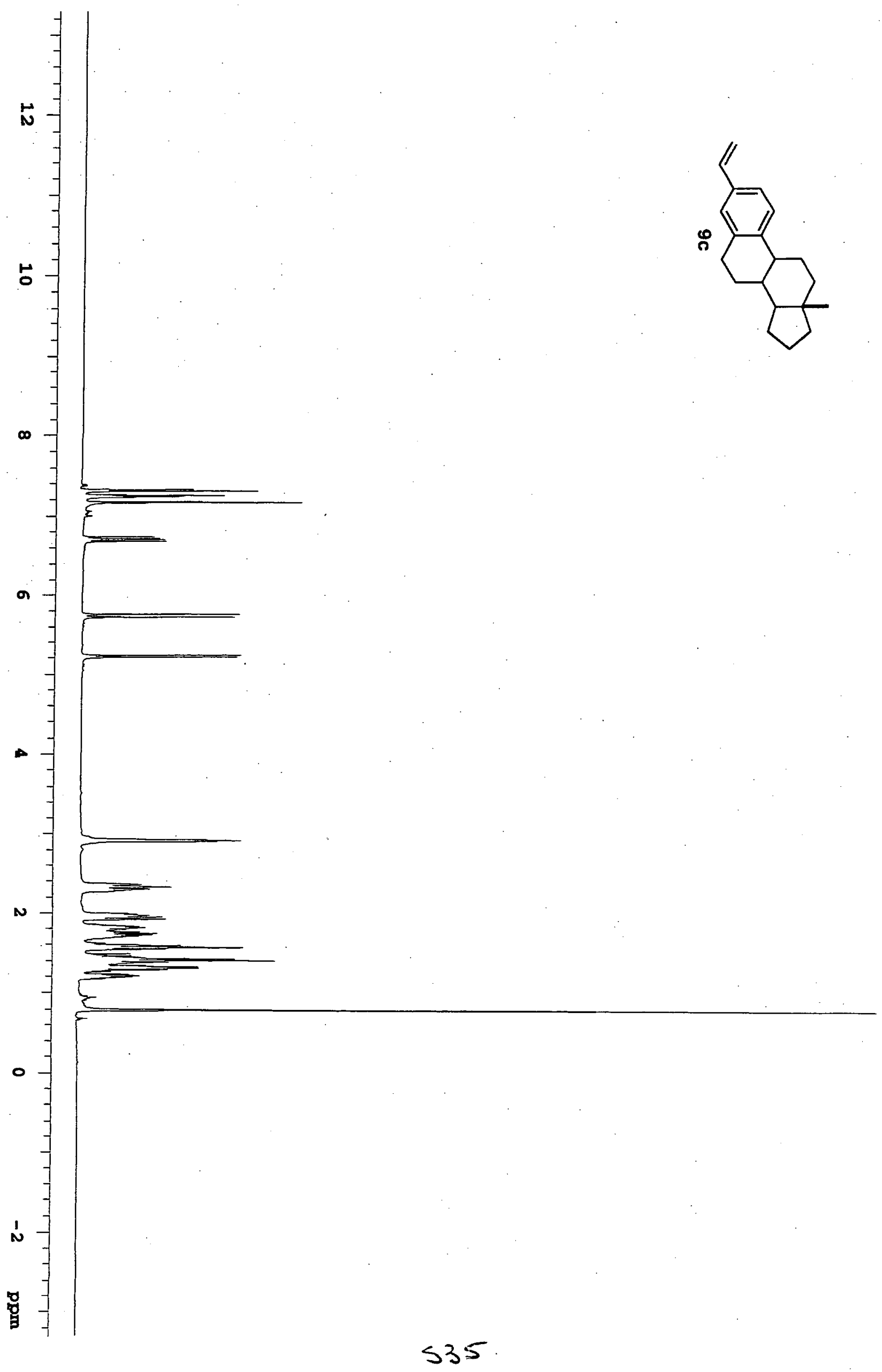


(C) 2004 American Chemical Society, J. Org. Chem., Postema jo040254t Supporting Info Page 36

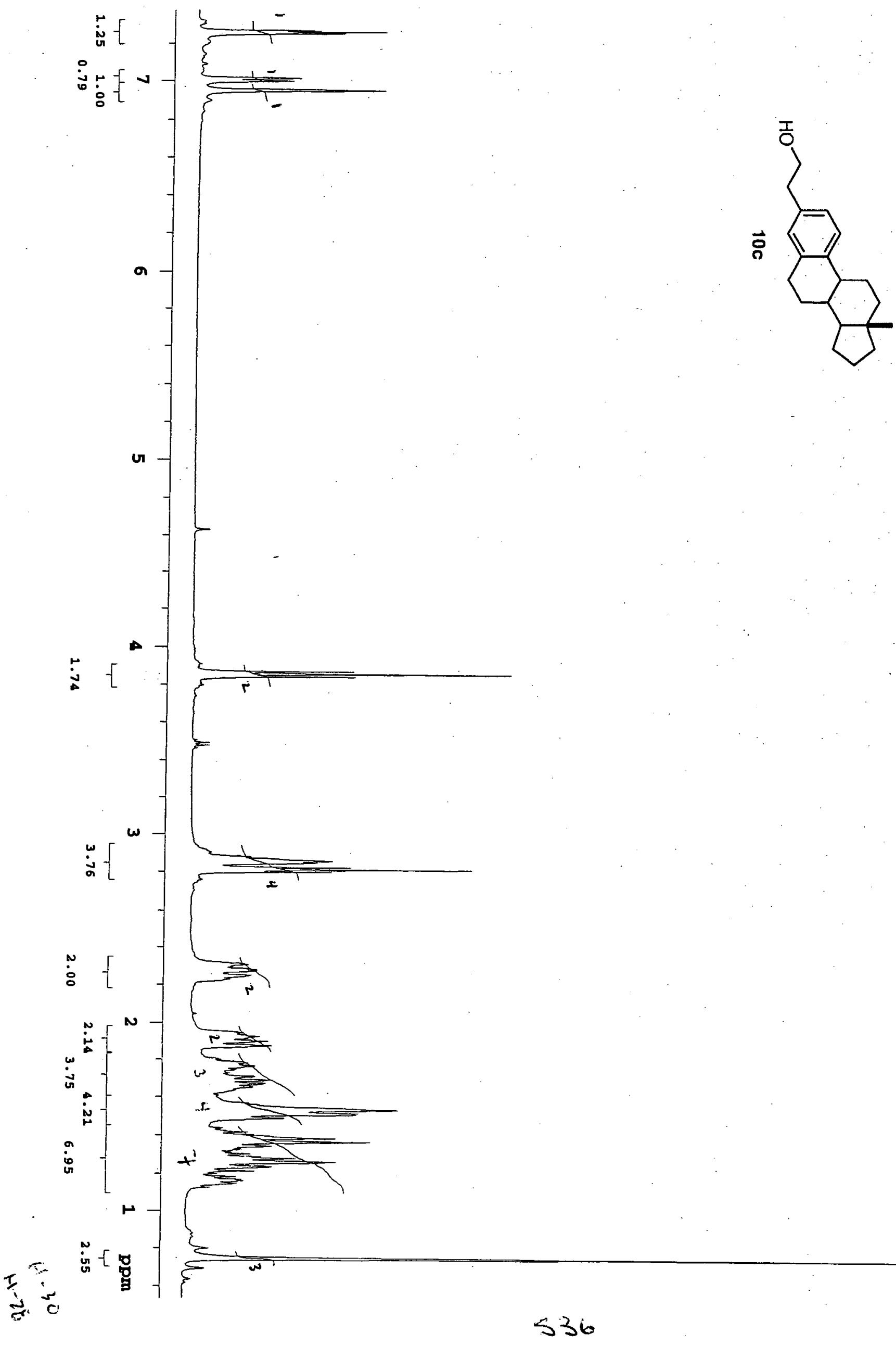


(C) 2004 American Chemical Society, J. Org. Chem., Postema jo040254t Supporting Info Page 37

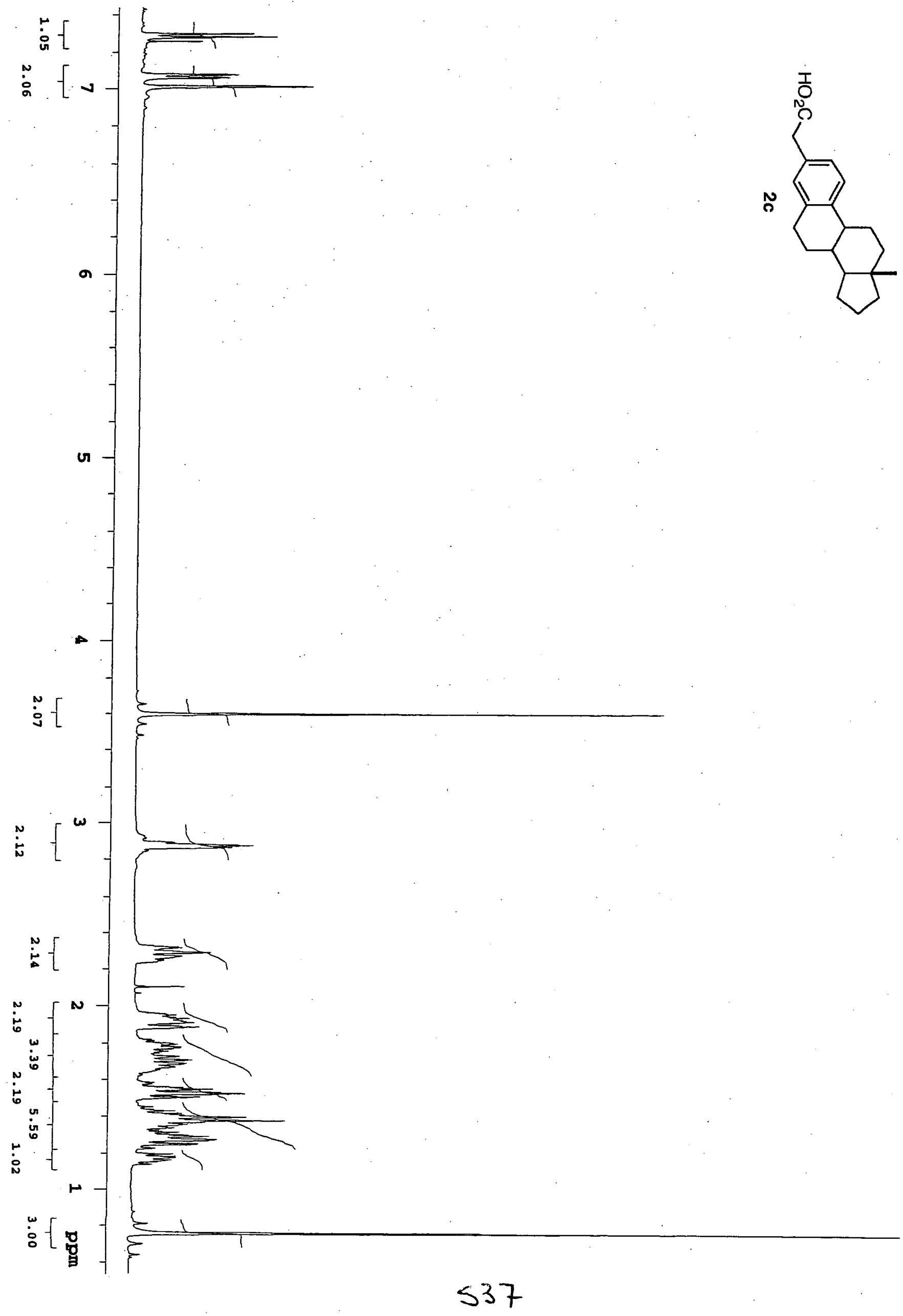


(C) 2004 American Chemical Society, J. Org. Chem., Postema jo040254t Supporting Info Page 38
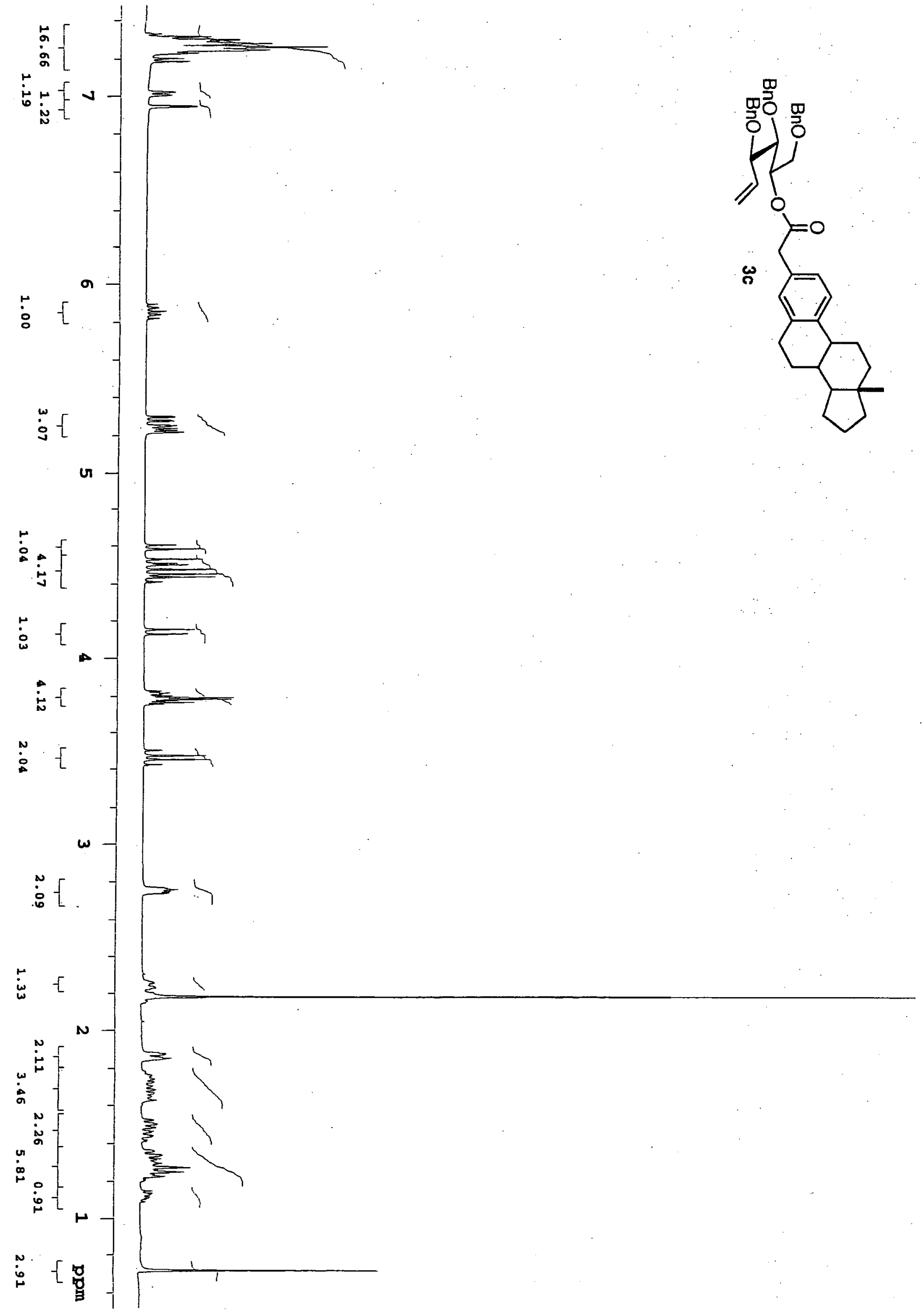
(C) 2004 American Chemical Society, J. Org. Chem., Postema jo040254t Supporting Info Page 39

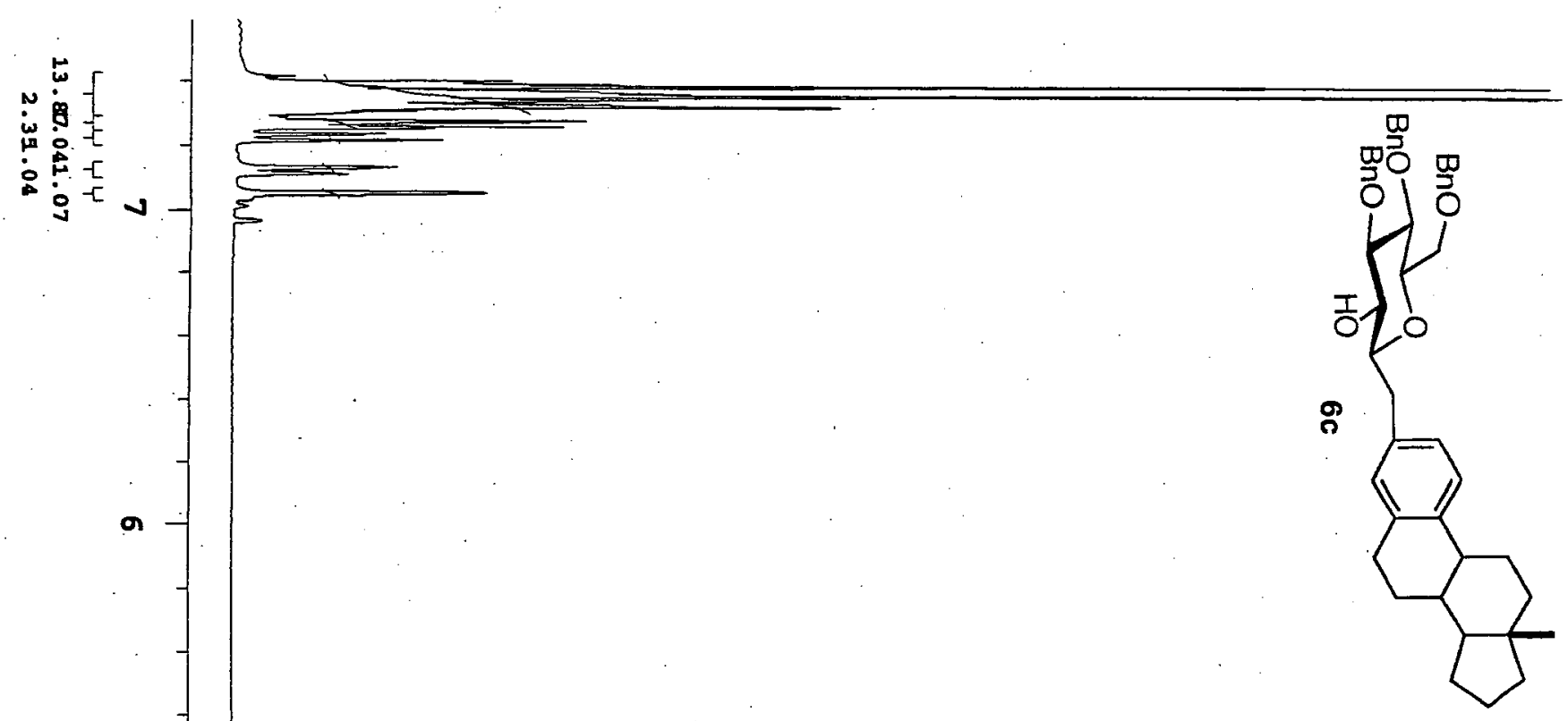


(C) 2004 American Chemical Society, J. Org. Chem., Postema jo040254t Supporting Info Page 40

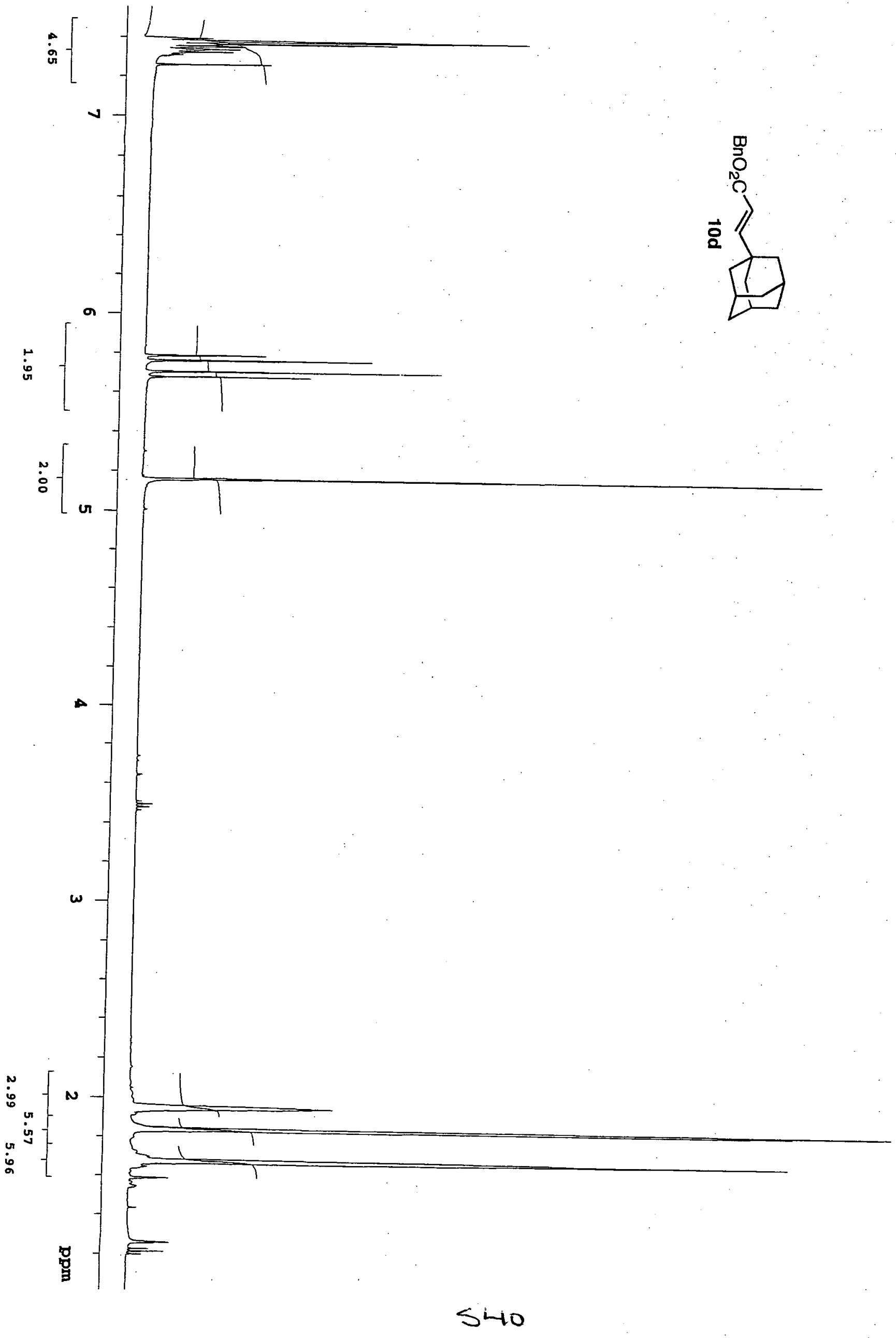

